\title{
Dynamics and Eigenfunctions of Hamiltonian Ratchets
}

\section{Dissertation}

zur Erlangung des Doktorgrades

der Mathematisch-Naturwissenschaftlichen Fakultäten

der Georg-August-Universität zu Göttingen

vorgelegt von

\section{Marc-Felix Otto}

aus Burgwedel

Göttingen 2002 
D7

Referent:

Koreferent:
Prof. Dr. Theo Geisel

Prof. Dr. Kurt Schönhammer

Tag der mündlichen Prüfung: 


\section{Contents}

1 Introduction $\quad 7$

2 Classical Dynamics in Hamiltonian Ratchets 13

2.1 Basics on Hamiltonian Systems _. . . . . . . . . . . . . . 14

2.1.1 Integrable Dynamics . . . . . . . . . . . . . . . 14

2.1 .2 The Poincaré Map . . . . . . . . . . . . . 15

2.1 .3 Mixed Dynamics . . . . . . . . . . . . . . 16

2.2 Directed Motion in Hamiltonian Ratchets . . . . . . . . . . . . . . 18

2.2.1 Definition of Hamiltonian Ratchets . . . . . . . . . . . . 19

2.2.2 A Typical Ratchet Model . . . . . . . . . . . . . 20

2.2.3 Asymptotic Velocities of Invariant Phase-Space Sets . . . . . . . . . 22

2.2.4 The Sum Rule for Transport . . . . . . . . . . . . . . 24

2.2.5 From Transport to the Asymptotic Particle Current . . . . . . . . 26

2.3 Kicked Maps as Model Systems for Ratchets . . . . . . . . . . . . 27

2.3.1 Symmetry Breaking for the Kicked Harper Map . . . . . . . . . 28

2.3.2 Deriving Ratchet Models from the Standard Map . . . . . . . . 29

2.3.3 Sum Rule for Compact Phase Space . . . . . . . . . . . . . . 33

2.4 The Rôle of Lévy Walks _ . . . . . . . . . . . . . . . . . . 33 
2.4.1 The Chain Model for Lévy Walks . . . . . . . . . . . . . . . . 34

2.4.2 Sum Rule for the Reduced Chaotic Set . . . . . . . . . . . . 35

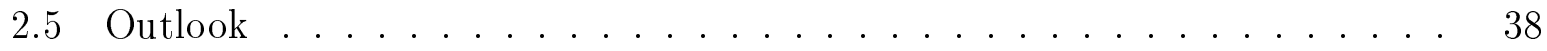

3 Perfect Quantum Hamiltonian Ratchets 39

3.1 Quantization of Hamiltonian Ratchets ............... 40

3.1 .1 Periodicity in Time . . . . . . . . . . . . . . . . . . 40

3.1.2 Periodicity in Space and Momentum . . . . . . . . . . . . . 41

3.1.3 Quantum Phase-Space Representations . . . . . . . . . . . . . . 43

3.1.4 Regular and Chaotic States . . . . . . . . . . . . . . 44

3.2 Reading Transport from the Slopes of Floquet Bands . . . . . . . . . . . 44

3.2.1 The Quantum Sum Rule for Transport . . . . . . . . . . . . . 45

3.2 .2 Asymptotic Wavepacket Transport . . . . . . . . . . . . . . 47

3.2 .3 Dynamical Tunneling . . . . . . . . . . . . . . . . 5 50

3.3 Short-Time Transport and the Generalized Form Factor . . . . . . . . . . 53

3.4 Chern Indices for Perfect Ratchets . . . . . . . . . . . . 55

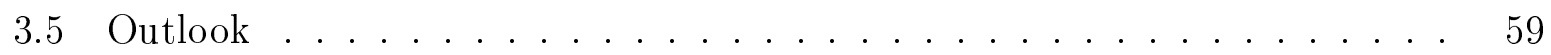

4 Disordered Quantum Hamiltonian Ratchets $\quad 61$

4.1 Dynamical Localization . . . . . . . . . . . . . . . . . . 61

4.2 The Semiclassical Eigenfunction Hypothesis . . . . . . . . . . . . 64

4.3 Broken Time-Reversal Symmetry . . . . . . . . . . . . . . . 65

4.3.1 Amphibious Eigenstates . . . . . . . . . . . . 65

4.3.2 Local Probability Current . . . . . . . . . . . 68

4.3.3 Perturbation Theory of Mixed Phase Space . . . . . . . . . . . 70

4.4 Time-Reversal Symmetric Case . . . . . . . . . . . . . . . . . . 70 
4.4.1 Eigenstates in the Symmetric Case . . . . . . . . . . . . . 71

4.4.2 Accessing the Critical Parameters with a Graph Model . . . . . . . 73

4.5 Wavepacket Dynamics in Disordered Hamiltonian Ratchets . . . . . . . . 79

4.5.1 Limit for Transport in Disordered Ratchets . . . . . . . . . . . . 79

4.5.2 Dynamics at the Wave-Packet Front . . . . . . . . . . . 81

4.5.3 Asymptotic Wave-Packet Shape . . . . . . . . . . . . . . 81

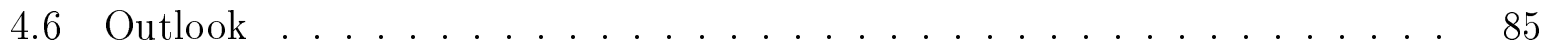

$\begin{array}{llr}5 & \text { Conclusion } & 87\end{array}$

A Generalization of the Hellman-Feynman Theorem $\quad 91$

A.1 Hellman-Feynman Theorem for Time-Periodic Systems . . . . . . . . . . 91

A.2 The Unitary Transformation of the Kinetic Energy _ . . . . . . . . . 92

B Asymptotic Wavepacket Transport $\quad 95$

C Generalized Form Factor for Periodic Maps in the Semiclassical Limit 97

C.1 Generalized Form Factor for a Regular Island _ . . . . . . . . . . . . 97

C.2 Form Factor for the Chaotic Component . . . . . . . . . . . . . 98 


\title{
Chapter 1
}

\section{Introduction}

\author{
ratchet \\ 1: a mechanism that consists of a \\ bar or wheel having inclined teeth into \\ which a pawl drops so that motion can \\ be imparted to the wheel or bar, gov- \\ erned, or prevented and that is used \\ in a hand tool (as a wrench or screw- \\ driver) to allow effective motion in one \\ direction only \\ Merriam-Webster Collegiate Dictio- \\ nary
}

The commonplace definition of a ratchet consists of two concepts, inclined teeth and motion in one direction, which, translated to broken symmetry and rectification, yield the physical definition. Ratchets, defined as systems which through some broken symmetry act as rectifiers, are ubiquitous in nature. In macroscopic mechanics, they serve as improved screwdrivers or self-winding wristwatches. On the molecular scale, motors like kinesin, responsible for the conversion of chemical energy into directed motion in biological cells [1-3], and particle separators [4], have been described in terms of a ratchet formalism. More recently, they even entered the quantum world through setups as diverse as antidot arrays [5], triangular quantum dots [6], Josephson-junction arrays passed by fluxons [7], surfaces supporting electromigration [8], cold atoms acted on by an asymmetric optical lattice [9], or in the improvement of superconductors by reduction of the vortex density $[10]$.

This wealth of experimental knowledge is accompanied by a long history of theoretical descriptions. The entity upon which the ratchet will act has to fulfill the condition of being non-rectified (otherwise, the ratchet would not be necessary) as is the case with an ensemble 
of particles subject to Brownian motion. Such a motion was described by Einstein [11] for a small particle in a liquid and independently by Langevin [12]. Smoluchowski [13] introduced the idea of a ratchet mechanism consisting of a potential with broken spatial symmetry that acts on the fluctuating particles. As Feynman [14] pointed out, however, the working of this ratchet for equilibrium fluctuations would violate the second law of thermodynamics. A decade ago, the investigation of molecular motors started to stimulate a lot of work on the effects of the presence of additional non-equilibrium forces [15-18]. The resulting concept was that of Brownian motors, nonlinear systems generating transport without an external bias. A first step in the reduction of this model was the replacement of the noisy fluctuations by deterministic chaos [19-22]. Following the exploration of rectification on the quantum level, Hänggi and collaborators [23-26] later studied the quantized Brownian motion of a damped particle which is subjected to an asymmetric external force. Recently, Flach et al. were able to observe the generation of a directed current by an alternating potential even in the absence of dissipation [27]. In their analysis they show that time-reversal symmetry has to be broken $[27,28]$, a requirement which is fulfilled in the previously known ratchets by the irreversibility of dissipation. However, they do not explain the observation, leaving the analysis of Hamiltonian ratchets, as minimal models for any ratchet, as an open problem.

Entering the realm of nonlinear Hamiltonian dynamics, one encounters an even longer history of theoretical research, starting with the formulation of Hamilton's equations of motion, and followed by a bulk of work on the solutions of linear equations. Although Poincaré showed that even a small perturbation of an integrable system can lead to the divergence of the perturbation series [29], it took about sixty years until Kolmogoroff [30] suggested that in this situation almost all regular tori in phase space are preserved, while the others are responsible for the onset of chaotic motion. This KAM theorem, consequently generalized by Arnold [31] and Moser [32], still represents the only solid mathematical basis for what subsequently became known as mixed phase space. Indeed, the generic [33] situation of regular and chaotic dynamics coexisting in phase space still awaits comprehensive understanding. Prominent among the models for investigating such systems are canonical mappings, as exemplified by the standard map. Introduced by Chirikov [34], it has become the paradigm for classical Hamiltonian systems in general, and for the observation of the transition from integrability to chaoticity under the change of only one parameter in particular. For certain values of this parameter, the standard map exhibits so-called accelerator modes of constantly increasing momentum. Together with the intrinsic properties of a mixed phase space, this leads to the phenomenon of Lévy walks [35], sections of ballistic chaotic motion the length distribution of which decays algebraically. Timereversal symmetry, however, renders the standard map useless for the exploration of the ratchet mechanism since for each trajectory there is a symmetry-related counterpart which compensates transport. The more general situation where this symmetry is broken has not been considered so far.

The desire to realize ratchets in nanoscale systems [6] requires the investigation of the consequences of quantization. Whereas the integrable case, as in classical mechanics, has 
received a lot of attention due to its comparative simplicity, the discipline of quantum chaos still poses many questions. Indeed, as the uncertainty principle prevents infinitesimally small distances in phase space, and thereby intrinsically violates sensitivity which in turn defines chaos, quantum mechanics is always "regular". However, classical chaoticity leaves its fingerprints in the quantum domain. Up to now, most of the work has been done for systems whose classical counterpart is fully chaotic. For the semiclassical limit, i.e., the typical wavelength of the system approaching zero, Bohigas, Giannoni, and Schmit [36] showed that the statistics of eigenenergies correspond to that of the eigenvalues of random matrices as introduced by Wigner, Dyson and Metha [37] two decades previously. This random matrix theory represents one of the cornerstones of quantum chaos. For the same limit, Gutzwiller [38] was able to relate the energy spectrum of the quantum system to the actions of periodic orbits of its classical version by the famous trace formula. Among the various works stimulated by this theory, Berry's theory of the form factor of a chaotic system is of particular interest here since it can be generalized to yield insights into the diffusive transport properies of extended systems [39].

Like random matrix theory, the trace formula is another fundamental result of quantum chaos, however, it does not account for mixed systems either. This gap is closed by a third cornerstone, the semiclassical eigenfunction hypothesis. Percival [40] was the first to consider the energy spectrum of such systems as a combination of two subsets, a "regular" and a "chaotic" one, each of which satisfies the statistical properties of the respective limit. In another fundamental work, Berry [41,42] and later Berry and Robnik [43] transferred this concept to the eigenstates of the Hamiltonian, stating that almost all of them are either regular or chaotic, in the sense that they are confined to the corresponding regions in phase space. Though stated explicitly only for the semiclassical limit, the succeeding numerical and experimental data [44-46] yielded strong support to the assumption that most eigenstates are confined to the invariant classical phase-space regions as soon as the de Broglie wavelength is small enough to resolve them. In a thought experiment, one can think of continuously decreasing the wavelength of the system. According to the observations under such a decrease, the theory has also been named (semiclassical) condensation hypothesis. Ketzmerick et al. [47] further extended the concept to the small classically fractal regions, presenting states condensated on them.

Maybe the most fascinating transport phenomenon discovered in quantum chaotic or disordered systems is localization. While disordered systems in the macroscopic regime support diffusion for all times, the quantum analogues behave as if they were confined by an exponentially declining envelope which prevents the infinite spreading of density. This counterintuitive observation, leading to insulating properties of the sample, is of major relevance for solid state physics. The first theoretical explanation was given by Anderson $[48,49]$ for the motion of a particle on a lattice of sites subjected to a random potential. Fishman, Grempel, and Prange [50] related the standard map to this Anderson model, thereby opening the field of localization to the study of canonical mappings. However, this requires that the spatial period be no integer multiple of the quantization parameter. The 
violation of this requirement leads to periodic extended Bloch states. Raizen et al., by exposing cold sodium atoms to standing-wave light fields [51-53], transferred the theory to experiments. Although dynamical localization in diffusive systems had been studied at length (for a review, see [54]), it remained an open question how ballistic transport, caused, for example, by accelerator modes, modifies the process. Only recently, investigations have been started in this direction, and a huge widening of the localization barriers was observed [55-58]. Again, Raizen et al. realized a corresponding experiment [59]. Lacking an explicit description for the observation, however, one could neither predict the length scale of this widening nor explain correctly the emergence of an intermediate algebraic regime.

In this thesis the mechanism is explained that leads to directed chaotic transport in Hamiltonian systems with spatial and temporal periodicity, allowing the construction and optimization of dissipationless ratchets. These systems are quantized for the first time. In disordered quantum Hamiltonian ratchets, a new class of eigenstates is found which challenges the semiclassical eigenfunction hypothesis: it is argued that even in the semiclassical limit the states are not confined to the classical phase-space regions. The analysis of eigenstates both in quantum systems with exact periodicity and in those with disorder leads to the understanding of the surprising dynamical properties and clarifies how ballistic transport and localization are reconciled. Therefore, while inspired by applications, this thesis makes several contributions to the theory of quantum chaology.

The thesis is structured as follows:

In the second chapter an outline of the foundations of Hamiltonian chaos is given, followed by the definition of Hamiltonian ratchets. The rectification mechanism in the absence of dissipation is shown to depend crucially on the phase-space structure, leading to a sum rule for ballistic transport [60]. Based on the insight obtained from this sum rule the class of model systems can be introduced [61]. Breaking time-reversal symmetry in canonical maps, a method for the construction of efficient ratchets is given. By following this instruction, various ratchet models are established and investigated throughout the thesis. Finally, the generation of transport by Lévy walks, which has been assumed to provide a sufficient explanation for the mechanism, is shown to be superseded by the sum rule.

The third chapter introduces quantum Hamiltonian ratchets obeying Bloch theory, again starting with a sketch of the foundations required. Based on a new interpretation of the Hellman-Feynman theorem for Bloch bands of quasienergies, a quantum sum rule analogous to the classical one is established [60]. The analysis of these bands and the corresponding eigenstates solves the paradox of non-trivial transport persisting in the presence of quantum tunneling for infinite times. The form factor is calculated from the Bloch bands in respect of their relevance for quantum Hamiltonian ratchets, followed by the analysis of a second spectral quantity known to indicate transport, the Chern number [62].

In the fourth chapter the condition of perfect periodicity is abandoned, raising the question of the compatibility of transport with localization. The new and completely unexpected amphibious eigenstates are presented [63], which contradict the semiclassical eigenfunction hypothesis. By explaining these states a criterion for the applicability of the 
semiclassical eigenfunction hypothesis is obtained. For systems with time-reversal symmetry, namely those analogous to the standard map in the regime of accelerator modes, the eigenstates are calculated for the first time. They too exhibit interesting new properties. The new insight into such systems can solve the problems of wave packet dynamics in the presence of accelerator modes.

The fifth chapter concludes by summarizing the main results of the thesis. 


\section{Chapter 2}

\section{Classical Dynamics in Hamiltonian Ratchets}

This chapter is concerned with the description of dissipationless ratchets in the classical limit. By investigating the phase-space structure of such systems it can be explained how they can make particles initially at rest move at a predetermined velocity.

The first section represents a brief introduction to the field of classical Hamiltonian mechanics as required for the understanding of the ratchet mechanism. A more comprehensive treatment of these basics can be found, for example, in [64,65]. These fundamentals are sufficient to define Hamiltonian ratchets and explain the ratchet mechanism in the second section [60]. Based on the definition of ballistic transport in terms of phase-space regions, the main result is the sum rule for classical transport. This sum rule shows that directed transport is a property associated with the individual invariant sets of the dynamics. A necessary condition for non-zero chaotic transport is a mixed phase space with coexisting regular and chaotic regions. Transport in chaotic regions can be described quantitatively by analyzing the adjacent regular regions. The relevance of this insight is made more explicit in the third section which deals with the construction and optimization of Hamiltonian ratchets. In the last section, it is shown that Lévy walks are not required for the generation of directed chaotic motion. 


\subsection{Basics on Hamiltonian Systems}

\subsubsection{Integrable Dynamics}

Although they are very rare in nature, integrable systems have received most attention in the past. This stems from the fact that only they can be solved analytically.

Hamiltonian systems are characterized by the fact that the whole dynamical information can be extracted from a single function. This function is denoted by $H$, the Hamiltonian of the system, which can depend on time and on the generalized position and momentum variables, $x_{i}$ and $p_{i}$, which satisfy the Hamilton equations of motion,

$$
\dot{x}_{i}=\frac{\partial H}{\partial p_{i}}, \quad \dot{p}_{i}=-\frac{\partial H}{\partial x_{i}} .
$$

For these equations to be integrable, the following conditions must be satisfied. First, the system has $n$ independent constants of motion $F$ with $\{F, H\}=0$, where $n$ is the number of degrees of freedom and the Poisson brackets are defined by

$$
\{u, v\}=\sum_{k}\left(\frac{\partial u}{\partial x_{k}} \frac{\partial v}{\partial p_{k}}-\frac{\partial v}{\partial x_{k}} \frac{\partial u}{\partial p_{k}}\right) .
$$

For time-independent Hamiltonians, the energy $E$ is such a constant of motion, leaving $n-1$ other constants to be found (hence, in the case of one dimension, each timeindependent system is integrable). Second, the Poisson brackets of these functions must vanish $\left\{F_{i}, F_{j}\right\}=0 \forall i \neq j$. The expression of $H$ in terms of the functions $F_{i}$ can then be achieved by the transformation to action-angle variables, leaving only $n$ independent variables of $H$.

The phase space is spanned by the $2 n$ position and momentum variables (and time $t$, if $H=H(t)$, see below). A point in phase space completely determines the subsequent motion. Following this motion yields a trajectory. In integrable Hamiltonian systems, up to a translation in time, a trajectory only depends on the $n$ variables of $H$, as stated above. For a compact phase space, this implies that the trajectory lies on a torus which is determined by a set of values of the constants of motion, the actions. A trajectory on such a torus can either be a periodic orbit, returning to the initial point after some time, or non-periodic. As trajectories in phase space may not intersect, each torus is filled either by periodic or non-periodic orbits. The first case corresponds to a rational ratio of angular frequencies of the trajectories, and the torus is called a resonant torus. With an irrational ratio, the torus is non-resonant and the corresponding trajectories are quasi-periodic orbits.

For the systems investigated in this thesis, the Hamiltonian is time-dependent, $H=$ $H(\mathbf{x}, \mathbf{p}, t)$. In this case, one can switch to the extended phase space $(\overline{\mathbf{p}}, \overline{\mathbf{q}})=(\mathbf{p},-H, \mathbf{q}, t)$ where $-H$ and $t$ are considered as conjugate variables. The new Hamiltonian is given by 
$\bar{H}(\overline{\mathbf{p}}, \overline{\mathbf{q}})=H(\mathbf{x}, \mathbf{p}, t)-E$ such that $\bar{H}=$ const. The dynamics of the time-dependent system is analogous to that of the time-independent one with an additional degree of freedom.

In a Hamiltonian system, a phase-space volume $\rho$ is preserved under the dynamics,

$$
\frac{\partial \rho}{\partial t}=\{H, \rho\}, \quad \frac{\mathrm{d} \rho}{\mathrm{d} t}=0 .
$$

This fact, known as Liouville's theorem, follows directly from the continuity equation for phase-space flows and Hamilton's equations [65].

\subsubsection{The Poincaré Map}

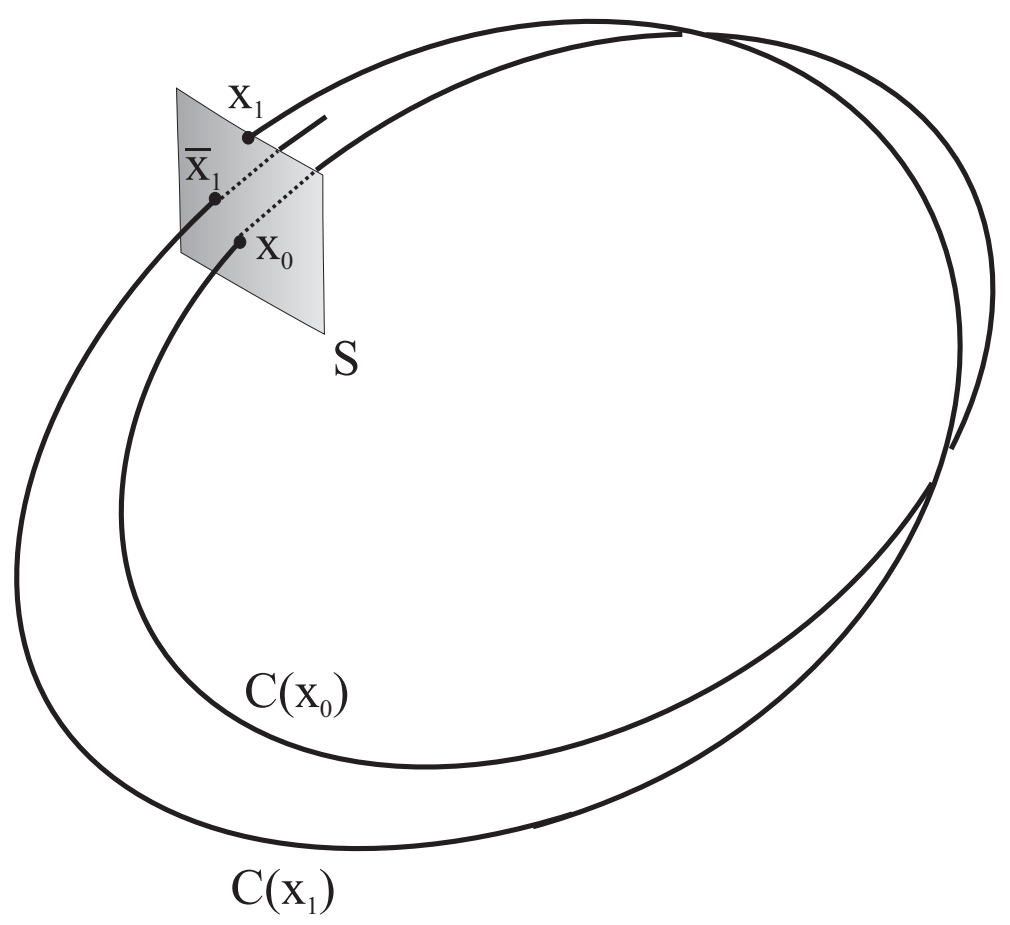

Figure 2.1: Poincaré surface $S$, intersected by a periodic orbit $C\left(x_{0}\right)$ yielding the fixed point $x_{0}$ of the map, and by a generic trajectory $C\left(x_{1}\right)$ leading to a sequence of points.

Commonly, the phase-space dimension is greater than two. In order to visualize such a phase space, one has to define a useful technique of projecting onto a lower-dimensional space. Such a projection is the Poincaré section, which is two-dimensional for a 3D phase space. It has to fulfill two conditions. First, the section must intersect all relevant trajectories and second, it must be transverse to the flow, i.e. no trajectory may be tangent to the section. An intersection of a trajectory with the surface will then lead to an sequence of intersections. The map which generates this sequence of points is denoted as Poincaré map (Fig. 2.1). For systems with a periodic time dependence, this section is naturally chosen 
at equidistant times $t=t_{0}+n \tau$, where $\tau$ denotes the time period. According to Liouville's theorem, the areas in such a stroboscopic section are preserved. A periodic trajectory leads to a finite set of points in the Poincaré section. A non-resonant torus yields an infinite set of points which appears as a (deformed) circle, while a chaotic trajectory (see Ch. 2.1.3) will produce a sequence of points spreading irregularly over the corresponding region in the projection plane.

\subsubsection{Mixed Dynamics}

For a generic system, the requirements for integrability are not met. The natural starting point then is perturbation theory. This approach has yielded deep insights into the onset of chaos. Let $H=H_{0}+\epsilon H_{1}$, with an integrable $H_{0}$ and a controllable perturbation parameter $\epsilon$. As stated by the KAM-Theorem, the first thing to happen under increasing $\epsilon$ is the destruction of the resonant tori, while the non-resonant tori are deformed slightly only. Within the set of real numbers, the set of rational numbers has zero measure. Therefore, almost all tori are preserved. For the further increase of the perturbation parameter, the theorem can often be expanded to the idea that "the more irrational a torus, the longer it survives": An irrational number $\alpha$ can be expressed in terms of a continued fraction expansion,

$$
\alpha=a_{0}+\frac{1}{a_{1}+\frac{1}{a_{2}+\ldots}}=\left[a_{0}, a_{1}, a_{2}, \ldots\right],
$$

with positive integers $a_{i}$. For a large $a_{i}, \alpha$ can be approximated with the rational number $\left[a_{0}, \ldots, a_{i-1}\right]$. The smaller the $a_{i}$ the worse this approximation. Therefore, the golden mean $\sigma$, with $a_{i}=1 \forall i$, is commonly denoted as the "most irrational number". Accordingly, the last $K A M$-torus to be destroyed is assumed to have a winding number ratio close to $\sigma$ [66].

The Poincaré-Birkhoff-Theorem states that a resonant torus situated between non-resonant tori with different directions of rotation in the integrable system decays to an even number of fixed points. The nature of these fixed points alternates between elliptic and hyperbolic. While the elliptic points are surrounded by a new generation of tori, the hyperbolic ones are responsible for the onset of chaos. This fact can be visualized using the homoclinic tangle Fig. 2.2. As soon as a set pointing to the fixed point (stable set) crosses the unstable set of the same point, a complicated structure of an infinite number of crossings emerges in the finite phase-space volume between the surrounding KAM-tori. Due to the infinite number of destroyed resonant tori, there is an infinite chain of tori of regular motion and chaotic layers between them. Within these layers, in turn, exist hierarchies of small islands. Increasing the perturbation even further, the phase space can be divided into a big unified chaotic sea and major regular islands of finite size within. Here, the notions of regular island and chaotic sea arise from the corresponding projection pictures, see Fig. 2.3. The torus separating the island from the chaotic sea is denoted as bounding torus or last KAMtorus, respectively. It is surrounded by a hierarchy of small islands. The destruction of 


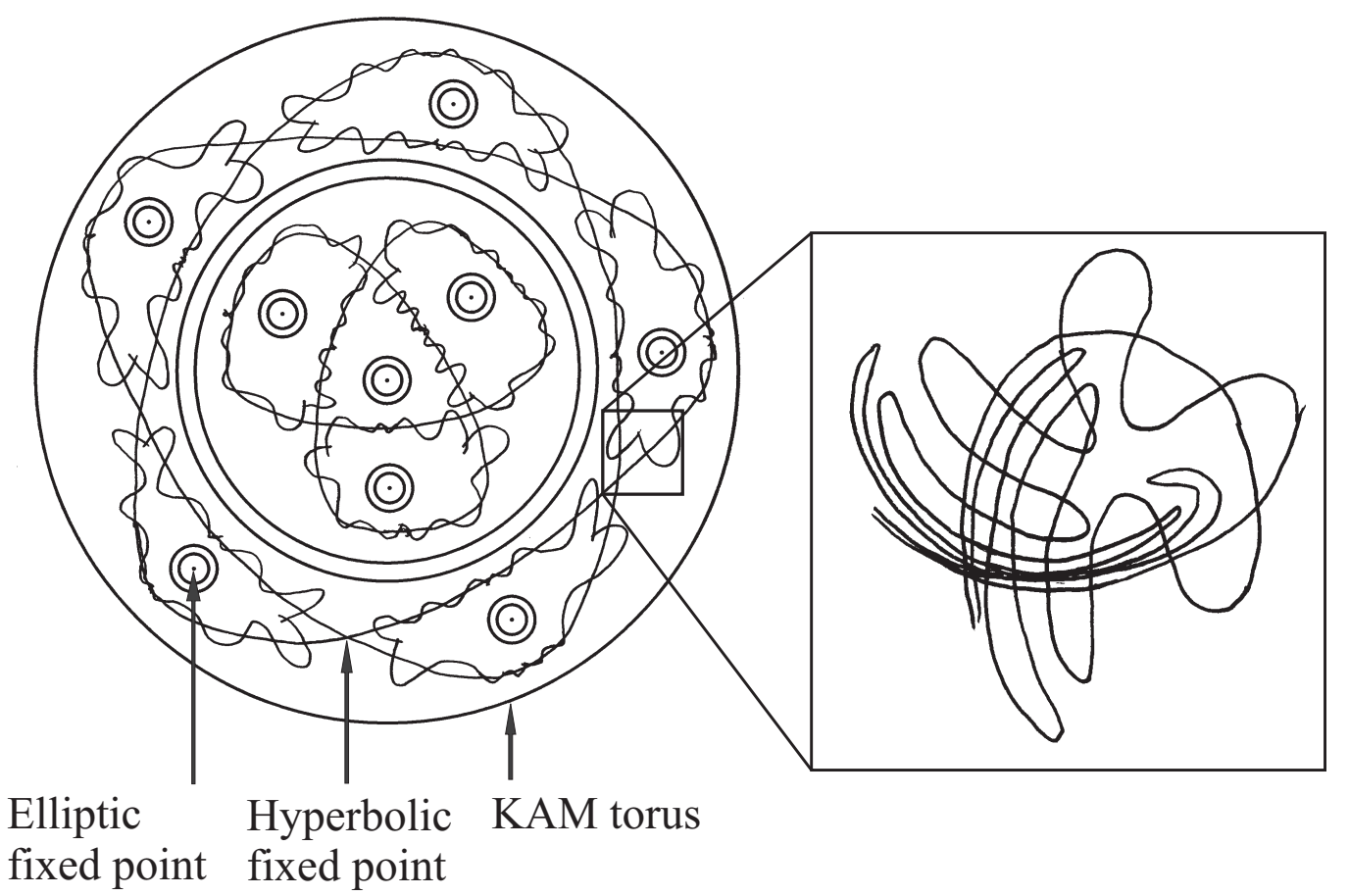

Figure 2.2: (left) Schematic view of a mixed phase space. Irrational KAM-tori are shown as circles surrounding elliptic fixed points. Sections of the stable and instable sets belonging to the same hyperbolic fixed point result in homoclinic tangles, a magnification of which is shown at the right.

KAM-tori, on the other hand, in the two-dimensional case leads to so-called cantori, partial phase-space barriers, which will be relevant in Ch. 2.4 .

For the purpose of this work it is very important to note that in one-dimensional time dependent systems, the two-dimensional bounding torus of an island generates a decomposition of the three-dimensional phase space, prohibiting trajectories to move from inside the torus to the outside. In terms of the stroboscopic section, a trajectory initialized on the island will always stay inside this island, whereas a trajectory initialized in the chaotic sea will never enter an island. This does not hold for higher-dimensional systems such as two-dimensional time dependent or three-dimensional autonomous ones. There, the three-dimensional tori do not separate regions in the five-dimensional phase space and the chaotic sea mixes with the regular islands through Arnold diffusion. 

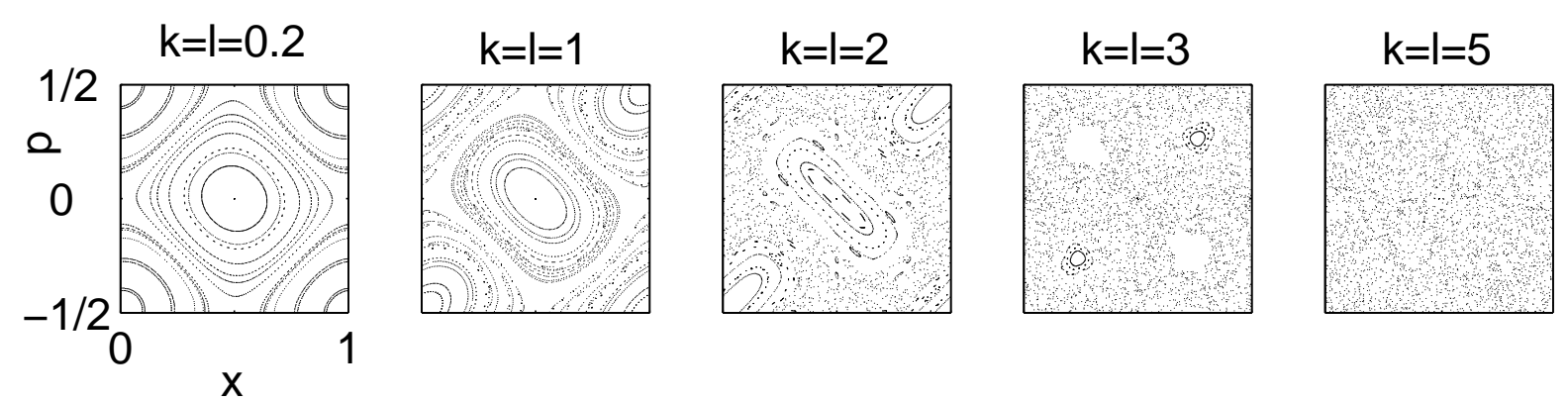

Figure 2.3: Transition from integrability to chaoticity. Shown are Poincaré sections of the kicked Harper map for different values of the parameters $k$ and $l$. The images were obtained by iterating 100 initial trajectories over 300 time periods. The choice $k=l$ leads to a phase space which is invariant under the exchange of position and momentum.

\section{Ergodicity of the Chaotic Dynamics}

Upon the decay of the last torus of regular motion, the phase space becomes fully chaotic. There may still be periodic trajectories but they have vanishing measure in phase space. For some sytems, it can then be shown for almost all initial points and a compact phase space, that the time mean of some observable equals the phase-space mean. For a onedimensional time-dependent system and with the observable $f$, this reads

$$
\langle f(x, p, t)\rangle=\lim _{t \rightarrow \infty} \frac{1}{t} \int_{0}^{t} \mathrm{~d} t^{\prime} f\left(x\left(t^{\prime}\right), p\left(t^{\prime}\right), t^{\prime}\right)=\int_{M} \mathrm{~d} \mu f(x, p, t),
$$

where $\mu$ denotes a measure in the phase space $M$. A system which obeys Eq. (2.5) is called ergodic.

For the chaotic components of mixed systems, ergodicity has not been proven. However, numerical observations give strong indications that it can rightfully be assumed.

\subsection{Directed Motion in Hamiltonian Ratchets}

This section contains the main result for classical dynamics, namely the sum rule for transport, which follows directly from the assumption of ergodicity and explains the origin of directed chaotic motion. The section starts with the specification of classical Hamiltonian ratchets, followed by the investigation of the average velocities of invariant phase-space sets. This leads to the notion of ballistic transport of finite invariant sets. Finally, it is shown how the results can be applied to calculate the time averaged current for some initial distribution of particles in phase space. 


\subsubsection{Definition of Hamiltonian Ratchets}

Throughout this thesis we consider infinitely extended one-dimensional Hamiltonian systems which are periodic both in space and time. The natural form of a Hamiltonian fulfulling these conditions is

$$
H(x, p, t)=T(p)+V(x, t),
$$

with

$$
V(x, t+T)=V(x+X, t)=V(x, t) .
$$

More precisely, the requirement that only the dynamics be periodic imposes a weaker condition on $V(x)$ : the equations of motion Eq. (2.1) depend only on the force $-V^{\prime}(x)$, not the potential itself. For example, it is allowed that the potential contains a term $x \cos t$. For notational simplicity, time and space are scaled such that $X=T=1$. Then the condition of spatial periodicity is expressed as $V^{\prime}(x+1, t)=V^{\prime}(x, t)$. To exclude trivial ballistic transport it is also required that the mean force $-V^{\prime}$ vanishes, i.e. $\int_{0}^{1} \mathrm{~d} t \int_{0}^{1} \mathrm{~d} x V^{\prime}(x, t)=0$. Due to the periodicity in time, the phase space is invariant under the transformation $t \rightarrow t+1$. The natural projection in this case is the stroboscopic Poincaré section at times $t=t_{0}+n T=t_{0}+n$.

Periodicity permits the effects investigated on small scales to be detectable on much larger scales: one can infer all the necessary information about the system from the study of one unit cell, i.e. a phase-space volume whose size is given by the periods. For example, an extended system cannot be ergodic since even in the long-time limit, a trajectory can never fill out the infinite phase space homogeneously. However, restricting the phase space to the cylinder which is obtained by associating the opposite borders of the unit cell, one can again assume ergodicity for the chaotic dynamics, which will prove valuable in the following sections.

The periodicities already define a Hamiltonian ratchet. However, systems which exhibit the interesting phenomenon of non-trivial directed transport of the chaotic sea satisfy another condition: those symmetries have to be broken which generate to each trajectory a second one moving in the opposite direction with the same velocity, see Fig. 2.4. For the Hamiltonian Eq. (2.6), this amounts to breaking time-reversal symmetry. The Poincaré section of a system with broken time-reversal symmetry does not have any point symmetry (see Fig. 2.4(bottom)) [27]. 

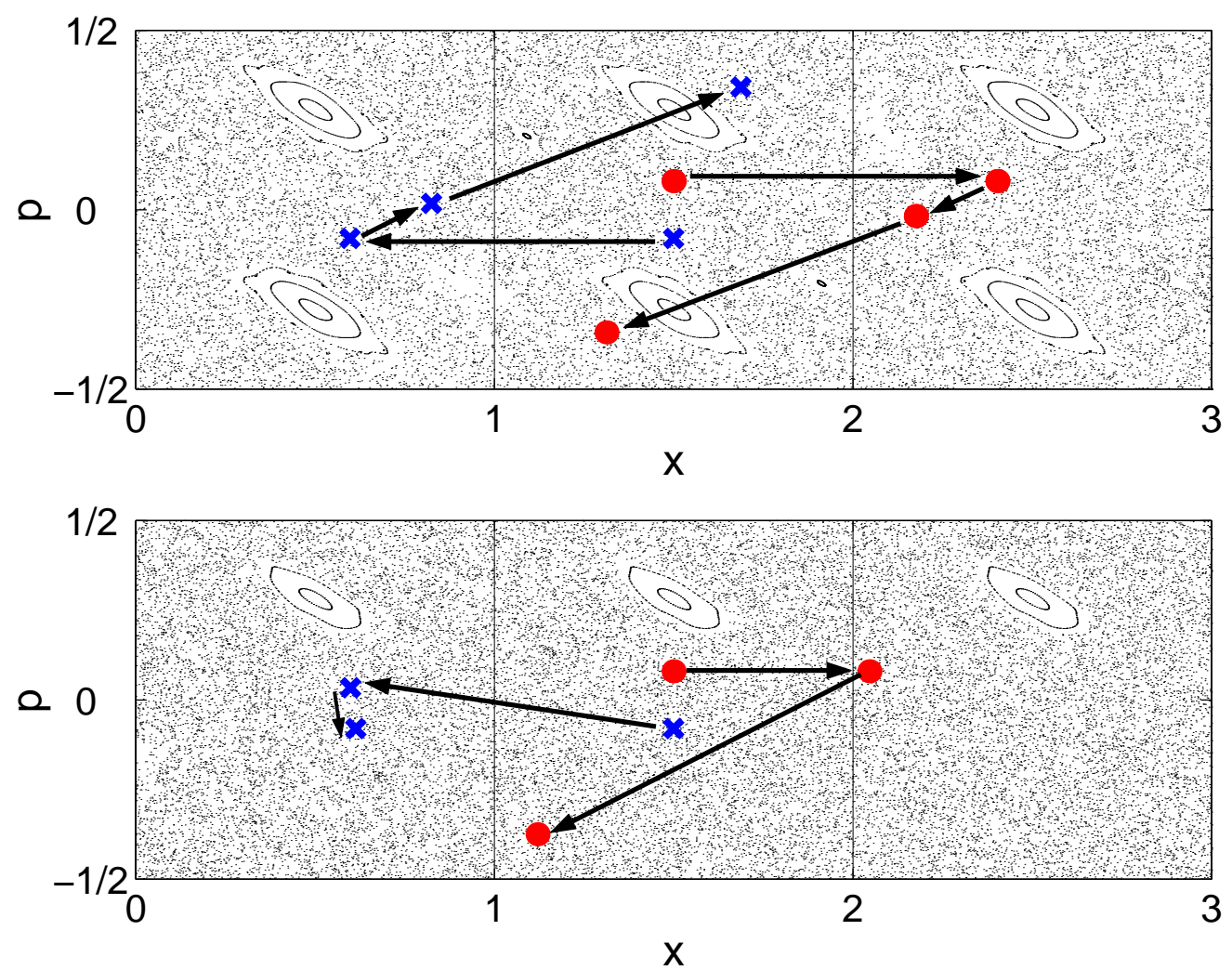

Figure 2.4: Spatial periodicity leads to a chain of identical unit cells in the stroboscopic section, only three of which are shown. The islands with positive (or negative, respectively) momentum are connected by the underlying torus. A trajectory will follow this chain of islands in the positive (or negative) direction. (top) System with time-reversal symmetry, which generates for each trajectory a countermoving partner: A trajectory with initial point $\left(x_{0}, p_{0}\right)$ is shown together with its partner starting at $\left(x_{0},-p_{0}\right)$.

(bottom) System with only one dominant island per unit cell and with broken symmetry. Such a system will exhibit non-trivial directed transport of the chaotic sea as soon as the island is transporting.

\subsubsection{A Typical Ratchet Model}

Fig. 2.5 shows the phase space representation of one unit cell of a typical ratchet, as studied by Flach et al. [27]. The Hamiltonian is given by $H=p^{2} / 2+V(x, t)$ with

$$
\begin{aligned}
\partial V(x, t) / \partial x= & \left(2 \pi / \omega^{2}\right)[\cos (2 \pi x)+0.6 \cos (4 \pi x+0.4) \\
& -2.3 \sin (2 \pi t)-1.38 \sin (4 \pi t+0.7)]
\end{aligned}
$$

Here, time-reversal symmetry is broken by the asymmetric time dependence of the potential, $V\left(x, t_{0}+t\right) \neq V\left(x, t_{0}-t\right), \forall t_{0}$. The interesting phase-space structure of this model will be studied in the following. 

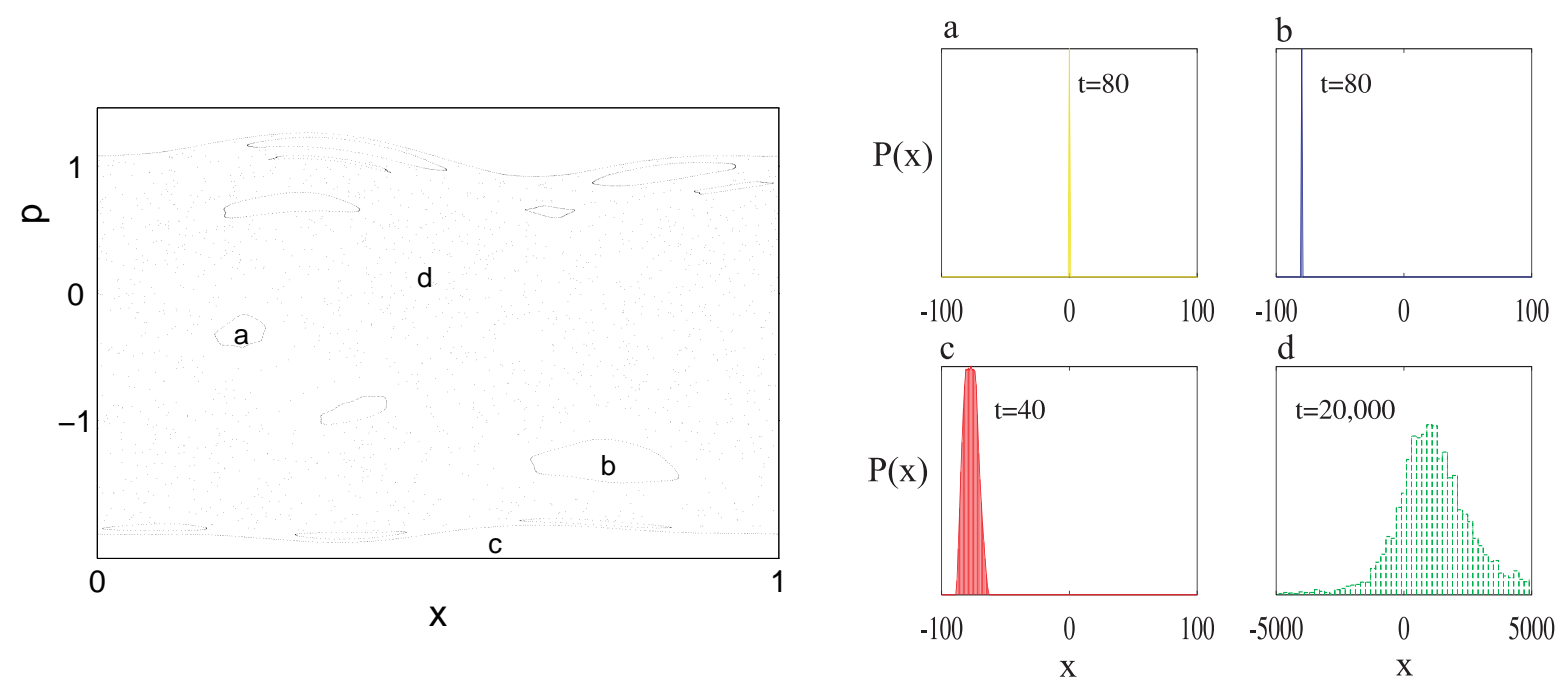

Figure 2.5: (left) Poincaré section of the intermediate layer and bounding extended tori of the ratchet model studied in [27]. The various regular islands transport at different velocities. (right) Spatial distributions $P(x)$ of trajectories initialized in a non-transporting island (a), in an island transporting in negative direction (b), on extended tori (c), the chaotic sea (d), after times as indicated.

In a finite mixed system without spatial periodicity, all the regular tori appear as deformed circles which enclose finite areas in a stroboscopic Poincaré section. In a Hamiltonian ratchet, spatial periodicity can lead to tori that in such a section appear as oscillating lines, closing only after a spatial period, see Fig. 2.5. The former are denoted as closed and the latter as extended or open tori. The physical meaning of the extended tori can be visualized using the simple model of a "comb potential" (see Fig. 2.6). The comb moving at small and constant velocity $v_{B}$ will grasp only those particles with a small initial velocity, setting their mean velocity to $v_{B}$. The fast particles, however, will not be influenced by the comb. In analogy, particles with a large initial velocity are affected only slightly by the ratchet potential, leading to dynamics like those of free particles under a small perturbation. In contrast, slower particles are affected more strongly such that their dynamics become more complicated. To be more precise, the situation can be compared to the transition from mixed to integrable dynamics as outlined in Ch. 2.1.3: For small absolute values of the momentum the phase space consists of a chaotic sea with embedded islands. With increasing $|p|$, one encounters extended tori, the first of which are denoted as (upper or lower) bounding torus. These extended tori are separated by small chaotic layers. Increasing $|p|$ even further, the extended tori become flatter and the intermediate layers thinner.

Obviously, the phase-space section also exhibits closed tori. The regular islands enclosed by such tori can be distinguished into two categories according to their dynamical properties. As Fig. 2.5 shows, trajectories initialized in island $a$ stay at their initial position while those in $b$ move in the negative direction. The latter are denoted as transporting islands, 


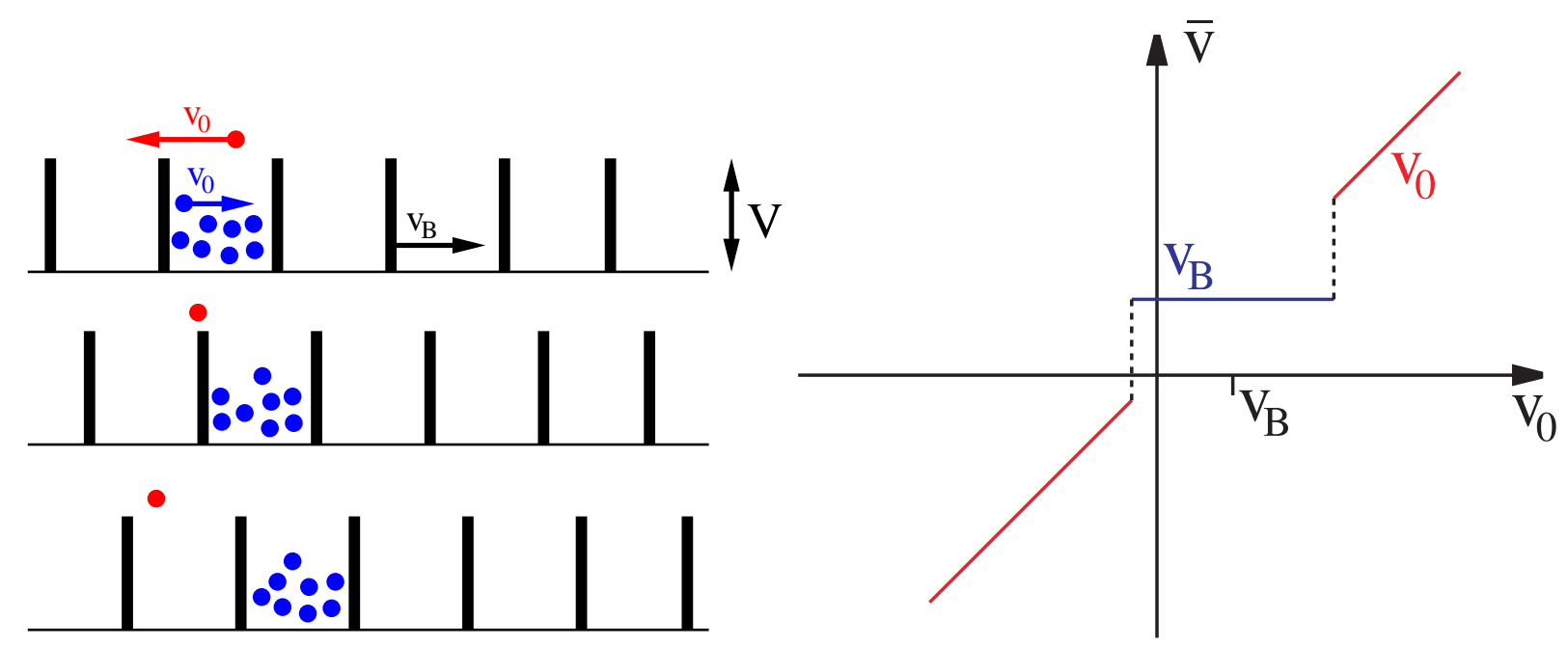

Figure 2.6: (left) Sketch of a "comb potential" with height $V$ moving at constant velocity $v_{\mathrm{B}}$. While those particles which have an initial velocity $v_{\mathrm{B}}^{2}-V<v_{0}^{2}<v_{\mathrm{B}}^{2}+V$ get caught, the others are not affected by the comb. In a typical system, the motion of the "caught particles" is chaotic or mixed, unlike in this simple example. (right) Mean velocity $\bar{v}$ as a function of the initial velocity $v_{0}$. The plateau corresponds to the particles grasped by the comb.

whereas island $a$ is non-transporting.

Finally, as Fig. 2.5(d) indicates, also those trajectories which were initialized in the chaotic sea show an average motion in one direction: on the one hand, the spatial distribution after 20.000 time periods is broadened due to the well-known phenomenon of chaotic diffusion. On the other hand, the mean is displaced from its initial position. The latter is the phenomenon of directed chaotic motion, to be explained in the next subsections.

\subsubsection{Asymptotic Velocities of Invariant Phase-Space Sets}

The standard method of demonstrating directed transport consists in calculating the average velocities of selected trajectories $[27,28]$ or of an ensemble of trajectories distributed over the corresponding phase-space region. In order to obtain a good average one has to calculate for very long times or large ensembles, which, given ergodicity within the chaotic component, is equivalent. The implementation of this procedure is straightforward but it gives no information about the origin of the transport. Instead, one can exploit the periodicity of the dynamics with respect to space and time, and analyze transport in terms of the invariant sets within one unit cell. The phase space of a typical ratchet exhibits three basically different types of minimal invariant sets: chaotic regions, non-resonant regular tori, and periodic orbits (which close on themselves after an integer multiple of the time period), with dimensions three, two, and one, respectively. According to Hamilton's 
equations (2.1), the instantaneous velocity of an arbitrary trajectory $C(t)=(x(t), p(t))$ is given by $v_{C}=\partial H(C, t) / \partial p$ and the long-time mean, with $\dot{x}=\partial H / \partial p$ Eq. (2.1), by

$$
v_{C, \infty}=\left.\lim _{t \rightarrow \infty} \frac{1}{t} \int_{0}^{t} \mathrm{~d} t^{\prime} \frac{\partial H}{\partial p}\right|_{C} .
$$

The calculation of this mean is the easiest for the periodic orbits. Due to periodicity in space and time, one can reduce the consideration to the spatio-temporal unit cell $(x, t) \in[0,1)$. Under the time evolution, the trajectory corresponding to a fixed point $i$ winds around a unit cell, returning to the initial point after some multiple of the time period. The number of (positive or negative) spatial windings divided by the number of time periods, that is the spatio-temporal winding number $w_{i}=x_{i} / t_{i}$, determines the long-time average velocity, $v_{i, \infty}=w_{i}$. This is valid both for hyperbolic and for elliptic fixed points. Whereas the dynamics in the vicinity of the former are chaotic, the latter are surrounded by closed tori. As mentioned in Ch. 2.1.3, these tori represent invariant barriers in phase space. Any trajectory initialized on the torus or on the enclosed phase-space volume will therefore always stay in the vicinity of the elliptic fixed point, with oscillations in the velocity around $w_{i}$ which are averaged out in the long-time limit. Therefore, all the minimal invariant sets which make up the island and its surrounding hierarchy of regular motion can be treated as a single (non-minimal) invariant set with an average velocity given by the velocity of the elliptic fixed point at the center of the island.

The investigation of the average velocity of a chaotic layer or of an extended torus, however, cannot be reduced to fixed points. Assuming that a trajectory initialized in the chaotic region (ch) fills out this region ergodically for large times, one can replace Eq. (2.9) with the average over the phase-space volume of $(c h)$. The latter, reduced to the spatio-temporal unit cell, reads

$$
v_{c h, \infty}=\frac{1}{V_{c h}} \int_{0}^{1} \mathrm{~d} t \int_{0}^{1} \mathrm{~d} x \int_{-\infty}^{+\infty} \mathrm{d} p \chi_{c h}(x, p, t) \frac{\partial H}{\partial p}
$$

where

$$
\chi_{c h}(x, p, t)=\left\{\begin{array}{lll}
1 & : & (x, p, t) \in c h \\
0 & : & (x, p, t) \notin c h
\end{array}\right.
$$

is the characteristic function and $V_{c h}$ the phase-space volume of $c h$,

$$
V_{c h}=\int_{0}^{1} \mathrm{~d} t \int_{0}^{1} \mathrm{~d} x \int_{-\infty}^{+\infty} \mathrm{d} p \chi_{c h}(x, p, t) .
$$

For completeness, one can also calculate the average velocity of an extended torus $a$. Since such a torus is a set of zero measure in phase space, one has to integrate $\partial H / \partial p$ over the torus to obtain the irrational winding number $w_{a}$. With a Hamiltonian of the standard form Eq. (2.6), the asymptotic velocity can then be calculated as

$$
v_{a, \infty}=w_{a}=, \int_{0}^{1} \mathrm{~d} t \int_{0}^{1} \mathrm{~d} x \frac{\partial H\left(x, p_{a}, t\right)}{\partial p}=\int_{0}^{1} \mathrm{~d} t \int_{0}^{1} \mathrm{~d} x \frac{\partial T\left(x, p_{a}, t\right)}{\partial p} .
$$


The use of $v_{a, \infty}$ instead of $w_{a}$ distinguishes the irrational winding numbers of extended KAM-tori from the rational ones of fixed points.

Since in the following only asymptotic averages will be of interest, the infinity index will be dropped, leaving $w_{i}, v_{a}$, and $v_{c h}$ as the denotions for the velocity of an island (or a hyperbolic fixed point), an extended KAM-torus, and the chaotic layer, respectively.

\subsubsection{The Sum Rule for Transport}

Besides the velocity, another quantity is introduced, in terms of which the dynamical properties of the system can be studied conveniently. Both the chaotic sea and the regular island are invariant sets of finite measure in phase space. For such a set $M$, ballistic transport is defined as phase-space volume times average velocity (cf. Eq. (2.10)),

$$
\tau_{M}=\int_{0}^{1} \mathrm{~d} t \int_{0}^{1} \mathrm{~d} x \int_{-\infty}^{+\infty} \mathrm{d} p \chi_{M}(x, p, t) \frac{\partial H}{\partial p} .
$$

Obviously, the characteristic function is additive for the union of disjunct invariant sets $M=\bigcup_{i} M_{i}$, namely $\chi_{M}=\sum_{i} \chi_{M_{i}}$. This additivity transfers to transport such that

$$
\tau_{M}=\sum_{i} \tau_{M_{i}}
$$

This is the sum rule for directed transport.

The extended tori enclose layers of chaotic motion with embedded regular islands. One can treat this layer as the global invariant set $\tau_{M}$ appearing on the 1.h.s. of Eq. (2.14). Assuming that $p_{a}=p_{a}(x, t)$, i.e. that the momenta on the bounding tori are functions of $x$ and $t$, its transport is obtained from Eq. (2.13) as the difference of the averaged kinetic energies of the bounding extended tori:

$$
\begin{aligned}
\tau_{\text {layer }} & =\int_{0}^{1} \mathrm{~d} t \int_{0}^{1} \mathrm{~d} x \int_{-\infty}^{+\infty} \mathrm{d} p \chi_{\text {layer }}(x, p, t) \frac{\partial H}{\partial p} \\
& =\int_{0}^{1} \mathrm{~d} t \int_{0}^{1} \mathrm{~d} x \int_{p_{l}(x, t)}^{p_{u}(x, t)} \mathrm{d} p \frac{\partial T}{\partial p} \\
& =\int_{0}^{1} \mathrm{~d} t \int_{0}^{1} \mathrm{~d} x\left(T\left(p_{u}(x, t)\right)-T\left(p_{l}(x, t)\right)\right) \equiv\left\langle T\left(p_{u}(x, t)\right)\right\rangle-\left\langle T\left(p_{l}(x, t)\right)\right\rangle,
\end{aligned}
$$

with indices $u$ and $l$ denoting the upper and lower bounding torus, respectively. Due to area conservation in the extended phase space and the fact that the time period equals 1 , the phase-space volume of a set of finite measure $M$ equals the area of this set in a stroboscopic section, $V_{M}=A_{M}=\int_{0}^{1} \mathrm{~d} x \int_{-\infty}^{+\infty} \mathrm{d} p \chi_{M}(x, p, t)$. One can therefore express the 
transport of the intermediate layer in terms of its components (chaotic sea and islands) as

$$
\tau_{\text {layer }}=\langle T\rangle_{u}-\langle T\rangle_{l}=A_{\mathrm{ch}} v_{\mathrm{ch}}+\sum_{i} A_{i} w_{i}
$$

(for an example, see Fig. 2.7). Solving for $v_{c h}$,

$$
v_{\mathrm{ch}}=\frac{\langle T\rangle_{u}-\langle T\rangle_{l}-\sum_{i} A_{i} w_{i}}{A_{\mathrm{ch}}}
$$

clearly indicates the origin of directed chaotic motion: If $\langle T\rangle_{u} \neq\langle T\rangle_{l}$, which is the general case if all symmetries are broken, one obtains a non-vanishing transport of the intermediate region. With asymmetric transporting islands, the chaotic transport can even exceed the value of the layer. Such regular islands can also generate directed chaotic motion if the boundaries are symmetric.
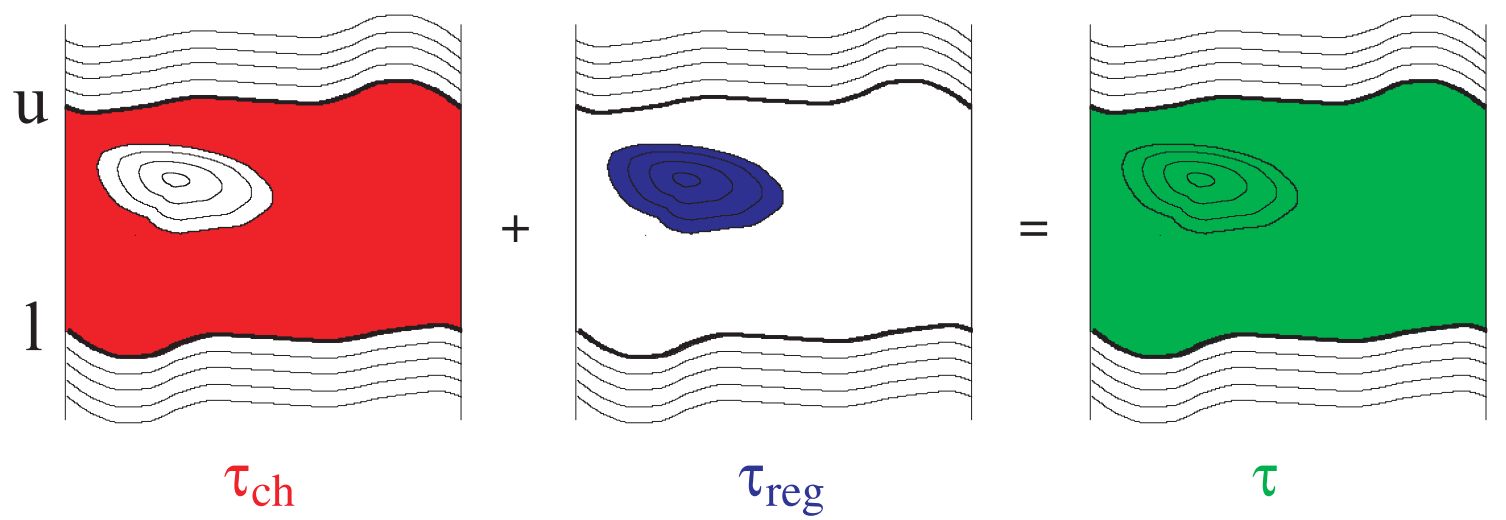

Figure 2.7: Sketch of the sum rule. The contributions to transport from the individual sets sum up to give the total transport of the intermediate layer. Therefore, the chaotic transport can be determined from the analysis of the regular components.

The only assumption needed for the derivation of this result is that the chaotic component is ergodic. Eq. (2.17) has been checked numerically for the continuously driven system given by Eq. (2.8). One can measure the area $A_{i}$ of an island by approximating its last torus with a polygon the vertices of which are given by the Poincaré section of some trajectory initialized on that torus. This area is multiplied with the winding number $w_{i}$ of the elliptic fixed point at the center of the regular island. Carrying out the procedure for all the islands visible in the Poincare section Fig. 2.5, as well as calculating the values $\left\langle T_{u}\right\rangle$ and $\left\langle T_{l}\right\rangle$ for the extended bounding tori, yields $v_{\mathrm{ch}}=0.092 \pm 0.011$. The error estimate includes the uncertainty in the location of the bounding tori and the contribution from neglected small islands. The result is in agreement with the value $v_{\mathrm{ch}}=0.082 \pm 0.002$, which was determined with much more computational effort from the spatial distribution of $10^{4}$ trajectories, started at the line $p=0$ (Fig. 2.8 a). For complex phase-space structures, the method of measuring island areas can be quite elaborate. However, it is a better controlled approximation than the traditional method of running long trajectories or large ensembles of trajectories. 

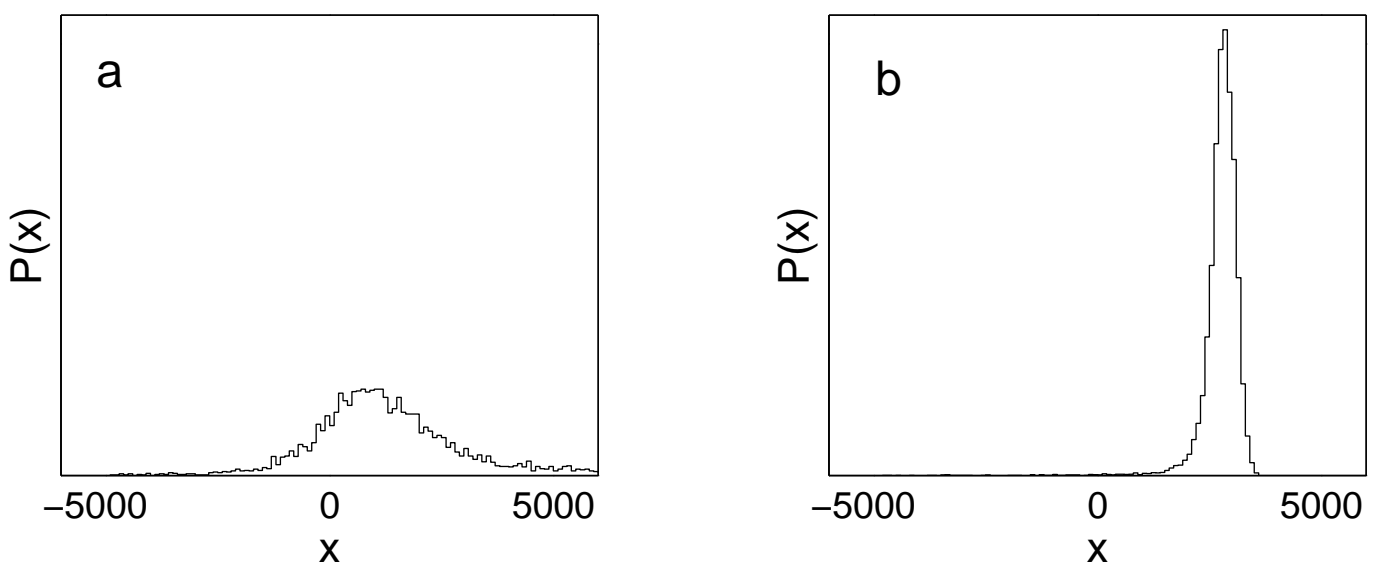

Figure 2.8: (a) Spatial distribution $P(x)$ of the ratchet system visualized in Fig. 2.5 after 20,000 time periods indicating the directed transport of the chaotic region. Initially, $10^{4}$ trajectories were started at random on the line $p=0, x \in[0,1)$ in the chaotic sea.

(b) Same as (a) but for the kicked Hamiltonian for Eq. (2.31) which is optimized to yield a much more pronounced transport.

\subsubsection{From Transport to the Asymptotic Particle Current}

The results for the asymptotic mean velocity of a trajectory will now be applied to calculate the asymptotic mean current both for a discrete initial distribution of particles in phase space and for a continuous distribution. First consider a discrete distribution at time $t_{0}=0$

$$
f_{\text {discr. }}(x, p, t)=\sum_{n} \delta\left(x-x_{n}\right) \delta\left(p-p_{n}\right) \delta(t)
$$

of particles $n=1,2, \ldots, N$. Here, the asymptotic mean current is simply the sum over the number of particles times the asymptotic mean velocities $v_{C_{n}, \infty}$ of the trajectories $C_{n}$ through the points $\left(x_{n}, p_{n}, 0\right)$,

$$
J_{f_{\text {discr }, \infty}}=\sum_{n=1}^{N} v_{C_{n}, \infty} .
$$

The velocities $v_{C_{n}, \infty}$ can be calculated according to sections 2.2.3, 2.2.4.

For a continuous and non-singular initial distribution $f(x, p, 0)=f(x, p), \int_{0}^{1} \mathrm{~d} x \int_{-\infty}^{+\infty} \mathrm{d} p f=$ $N$, the invariant sets of zero measure in phase space can be neglected and the current is determined by projection onto the finite sets. Since the shape of the distribution within an invariant set is irrelevant for the long-time average, it suffices to know the weight $\rho_{f, M_{i}}=\int_{M_{i}} \mathrm{~d} x \mathrm{~d} p f$ inside the stroboscopic projection of $M_{i}$ at the initial time $t_{0}=0$. The 
mean current is then given by

$$
J_{f, \infty}=\sum_{i} \rho_{f, M_{i}} v_{M_{i}, \infty}
$$

The method of projection onto invariant sets also applies to a distribution $f\left(x_{0}, p_{0}, t\right)$ smeared out over the temporal unit cell, that is, to the question of phase dependence of the current discussed in [67].

As mentioned in Ch. 1, a ratchet can only work far from the thermodynamic equilibrium. For a Hamiltonian ratchet, this condition is inherently fulfilled by the driving potential $V(t)$. However, it might be of interest to study the effects of a ratchet acting on particles initially in equilibrium as described by the Maxwell-Boltzmann distribution $f(x, p)=N \sqrt{\frac{a^{3}}{4 \pi}} f(x) p^{2} e^{-a p^{2}}$, where $f(x)=\int_{0}^{1} \mathrm{~d} p f(x, p)$, and $a>0$. Depending on the parameter $a$, such a distribution could either be concentrated in the intermediate layer of the system or spread over the extended tori as well. In the former case, one projects $f$ on the finite invariant sets. In the latter case, however, namely if the distribution is broad enough in momentum to have non-negligible weights on the extended tori, the situation is more complicated. Instead of calculating the average kinetic energy for each extended torus to obtain the stepwise increasing (or decreasing, respectively) asymptotic velocities of the small intermediate chaotic layers, one can make use of the comb potential picture introduced in Ch. 2.2.2. There, for large initial velocities $v_{0}$ the asymptotic velocity $\bar{v}$ is trivially given by $\bar{v}\left(v_{0}\right)=v_{0}$. Assuming perfectly flat extended tori $p_{a}(x, t)=$ const., this dependence is a good approximation for the part of the distribution beyond the bounding tori since it corresponds only to smoothing the small steps in $\bar{v}\left(v_{0}\right)$. For a ratchet with standard kinetic energy $T(p)=p^{2} / 2$ this yields for the asymptotic current

$$
J_{f\left(p>p_{u}\right), \infty}=\int_{p_{u}}^{\infty} \mathrm{d} p f(p) p
$$

where $f(p)=\int_{0}^{1} \mathrm{~d} x f(x, p)$, and analogous for the lower bounding torus. The approximation of perfectly flat tori improves for large kinetic energies. For a very broad non-biased thermal distribution, therefore, the average current is expected to vanish, in contrast to the case of a narrow distribution.

\subsection{Kicked Maps as Model Systems for Ratchets}

One is now in the position to specify the models used for the numerical studies of Hamiltonian ratchets. As Eq. (2.17) indicates, those systems generate directed chaotic transport which show a completely desymmetrized phase-space structure. With respect to the numerics, it is desirable to have in each unit cell only one large transporting first-order island with higher-order islands confined to a small neighborhood: First, this simplifies the sum 
over regular islands. Second, a large transporting island leads to large and therefore easily detectable directed chaotic motion. Third, a large island is numerically more easily dealt with also in quantum mechanics.

To calculate the time evolution of a trajectory in a system with a continuous time dependence like that given by Eq. (2.8), one has to integrate the equations of motion for each time period. This numerically expensive treatment can be avoided by studying systems with a discrete time dependence. In such systems, the potential acts at certain points in time (kicking) which for convenience are chosen equidistantly. The Hamiltonian can be expressed as

$$
H(x, p, t)=T(p)+V(x) \sum_{n=-\infty}^{+\infty} \delta(t-n), \quad n \in \mathbb{N},
$$

which yields a simple map

$$
p_{n}=p_{n-1}-V^{\prime}\left(x_{n-1}\right) \quad x_{n}=x_{n-1}+T^{\prime}\left(p_{n}\right)
$$

for the stroboscopic Poincaré section at times immediately before the kicking: The first equation represents the kicking whereas the second one describes the time evolution of a trajectory between two kicks. Since the potential between the kicks is zero, the latter is denoted as free time evolution. Due to the simplicity of such maps, the numerical investigations of the transition from integrability to chaos have often been carried out with them. Two prominent examples are introduced in the following, together with variants that serve as efficient models for the study of directed chaotic motion.

\subsubsection{Symmetry Breaking for the Kicked Harper Map}

The Harper map models the dynamics of conduction electrons in a uniform magnetic field [68-70]. The Hamiltonian is given by

$$
H=L \cos (p)+K \cos (x) \sum_{n=-\infty}^{+\infty} \delta(t-n) .
$$

According to Eq. (2.23), the stroboscopic map is obtained as

$$
p_{n}=p_{n-1}+K \sin \left(x_{n-1}\right), \quad x_{n}=x_{n-1}-L \sin \left(p_{n}\right) .
$$

The Harper map undergoes a transition from an integrable system at $K=L=0$ to a fully chaotic one at $K=L \approx 5$. Obviously, the Hamiltonian in Eq. (2.24) is periodic in $x$ and the phase space can be restricted to the cylinder $x \in[0,1)$. Moreover, the mapping Eq. (2.23) is invariant under the shift $p \rightarrow p+1$. Hence, the stroboscopic section can be restricted to the unit cell $(x, p)=[0,2 \pi)$. With

$$
H=\frac{L}{2 \pi} \cos (2 \pi p)+\frac{K}{2 \pi} \cos (2 \pi x) \sum_{n=-\infty}^{+\infty} \delta(t-n),
$$


this unit cell is scaled to $(x, p)=[0,1)$, as shown in Fig. 2.3.

The straightforward method for constructing a ratchet consists in breaking time-reversal symmetry in $V(t)$, either by two kicks with different strengths and different intermediate time intervals or by three different kicks equidistant in time. Choosing the latter option, one obtains a big transporting island with

$$
\begin{aligned}
V(x, t) & =\sum_{n=-\infty}^{+\infty} \delta\left(t-\frac{n}{3}\right) \times \begin{cases}K_{0} \cos (2 \pi x) & : 0.15 \leq(x+t) \bmod 1 \leq 0.85 \\
K_{1} \cos (2 \pi x) & : \text { else, }\end{cases} \\
T(p) & =L \cos (2 \pi p),
\end{aligned}
$$

where $K_{0}=0.15 / 2 \pi, K_{1}=5 / 2 \pi$, and $L=\frac{1}{3^{1.5} \pi}$ (see Fig. 2.10 b). The island around the elliptic fixed point at $(x, p)=(0,1 / 6)$ moves at the speed $w_{i}=-1$ to the left and is therefore always subjected to the small values of the kicking potential. The remaining phase space is chaotic due to the large kicking strength $K_{1} \gg K_{0}$.

\subsubsection{Deriving Ratchet Models from the Standard Map}

The kicked rotator models the dynamics of an object rotating in a plane without friction, hit periodically in a given direction with a strength depending on the angle and the kicking parameter $K$. Denoting the angle as $x$ and the angular frequency as $p$, the Hamiltonian is given by

$$
H(x, p, t)=\frac{p^{2}}{2}+K \cos (x) \sum_{n=-\infty}^{+\infty} \delta(t-n), \quad K \in \mathbb{R},
$$

or, with periods $X=P=1$, and $k=K / 2 \pi$,

$$
H(x, p, t)=\frac{p^{2}}{2}+\frac{k}{2 \pi} \cos (2 \pi x) \sum_{n=-\infty}^{+\infty} \delta(t-n),
$$

yielding the map

$$
p_{n}=p_{n-1}+k \sin \left(2 \pi x_{n-1}\right), \quad x_{n}=x_{n-1}+p_{n} .
$$

This model, introduced by Chirikov [34], received a lot of attention and is therefore called Chirikov or standard map. With kicking parameter $k=0$ it is obviously integrable, and it devolves to mixed and chaotic dynamics when $k$ is increased. Among the various domains of $k$ the most interesting one with respect to ballistic transport is $k=1+\epsilon$ with small $\epsilon$, respectively: For $k$ slightly larger than 1, the system exhibits accelerator modes at $p=m$, $k \sin (x)= \pm 1$, with integer $m$. These correspond to islands moving in $p$-direction, such that a trajectory initialized on them is accelerated (see Fig. 2.9). 
In order to convert the standard map into a ratchet, exchanging $x$ and $p$ in Eq. (2.30) yields a system with two first-order islands transporting at constant velocities $w_{i}= \pm 1$. This, of course, yields an unphysical kinetic energy. However, it is not required that $T(p)=p^{2} / 2$, or $\dot{x}=p$, respectively, for the study of the ratchet effect. Since in Hamiltonian systems momentum and position are related by Hamilton's equations (2.1), time-reversal symmetry is broken if $T(p) \neq T(-p)$. For the model system given by

$$
V(x)=0.5(x \bmod 1-0.5)^{2}, \quad T(p)=|p|+\frac{3}{4 \pi^{2}} \sin (2 \pi p),
$$

the dynamics can be restricted to the cylinder $p \in[-1 / 2,1 / 2)$ and the major island again transports at the velocity $w_{i}=-1$ (see Fig. 2.10 a) [60].

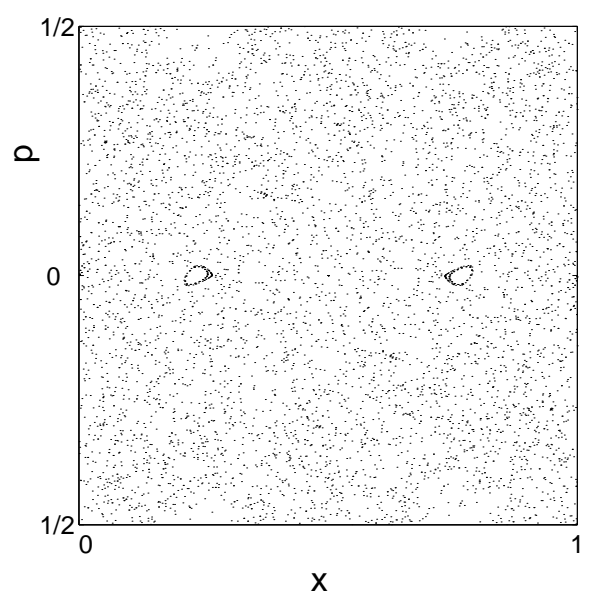

Figure 2.9: Poincaré section of the standard map with $k=6.33 / 2 \pi$. The two small accelerator modes at $x=(0.5 \pm 0.25), p=0$ transport in momentum direction at velocity $w_{i}=\mp 1$

\section{Phase-Space Engineering}

In order to enlarge the accelerator modes of the standard map with $k$ slightly larger than 1 it is useful first to analyze the stability of the fixed points at the centers of these transporting islands. The knowledge of the resulting stability criterion allows a straightforward optimization of the force $-V^{\prime}(x)$ under the restrictions of periodicity (cf. Ch. 2.2.1), and analyticity (a non- potential would later lead to quantum anomalies). Exchanging $x$ and $p$ transfers the optimized accelerator modes into islands transporting in $x$-direction.

The stability of a fixed point $\left(x_{0}, p_{0}\right)$ can be analyzed by linearizing the motion in the vicinity of that point [71]. Using vector notation $\mathbf{x}=\left(\begin{array}{l}x \\ p\end{array}\right)$, the standard map with generalized force reads $\mathbf{f}\left(\mathbf{x}_{n-1}\right)=\mathbf{x}_{n}$,

$$
\begin{aligned}
& f_{1}\left(\mathbf{x}_{n-1}\right)=p_{n}=p_{n-1}-V^{\prime}\left(x_{n-1}\right) \\
& f_{2}\left(\mathbf{x}_{n-1}\right)=x_{n}=x_{n-1}+p_{n-1}-V^{\prime}\left(x_{n-1}\right) .
\end{aligned}
$$


a

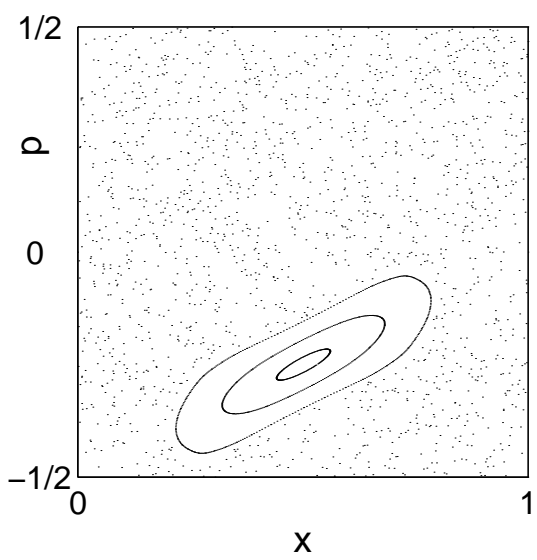

b

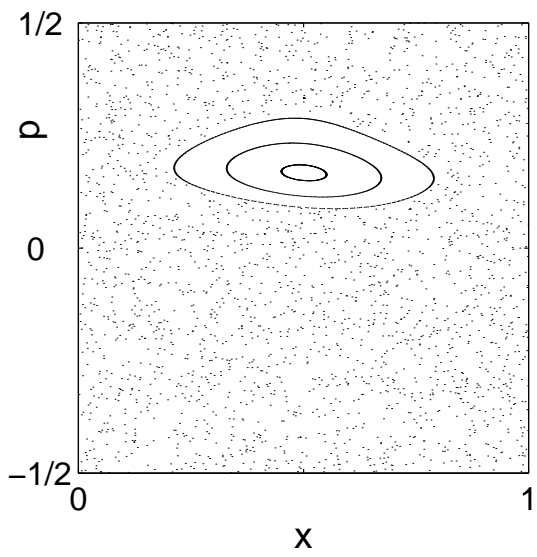

Figure 2.10: Poincaré sections of two paradigmatic ratchet models, both of which show a big island transporting with velocity $w_{i}=-1$. a) System derived from the Chirikov map, Eq. (2.31). b) System derived from the kicked Harper map with three kicks per time period, Eq. (2.27).

One considers the motion of some point $\mathbf{x}=\mathbf{x}_{0}+\Delta \mathbf{x}$ in the vicinity of the fixed point $\mathbf{x}_{0}=\left(x_{0}, p_{0}\right)$. The accelerator modes are period one fixed points, meaning that their trajectory closes after one time period in the restricted phase space. For such period one fixed points, keeping only the first order of the Taylor expansion of $f$ around $\mathbf{x}_{0}$ yields

$$
\Delta \mathbf{x}_{n} \approx \mathbf{A}\left(\mathbf{x}_{0}\right) \cdot \Delta \mathbf{x}_{n-1}
$$

where

$$
\mathbf{A}\left(\mathbf{x}_{0}\right)=\left(\begin{array}{ll}
\frac{\partial f_{1}}{\partial p} & \frac{\partial f_{1}}{\partial x} \\
\frac{\partial f_{2}}{\partial p} & \frac{\partial f_{2}}{\partial x}
\end{array}\right)_{\mathbf{x}_{0}}=\left(\begin{array}{cc}
1 & -V^{\prime \prime}\left(x_{0}\right) \\
1 & 1-V^{\prime \prime}\left(x_{0}\right)
\end{array}\right)
$$

is the Jacobian of $\mathbf{f}$ evaluated at the fixed point, and it is $\operatorname{det}(\mathbf{A})=1$. The eigenvalues $\lambda_{1,2}$ of $\mathbf{A}$ follow from the characteristic equation $\operatorname{det}(\mathbf{A}-\lambda \mathbf{I})=0$, or $\lambda^{2}-\lambda \operatorname{Tr} \mathbf{A}+1=0$. For a stable fixed point, the iteration of the mapping Eq. (2.33) may not cause the trajectory initially close to $\mathbf{x}_{0}$ to diverge from $\mathbf{x}_{0}$. This is fulfilled for an elliptic fixed point: There, the eigenvalues of $\mathbf{A}$ are complex conjugates $\lambda_{1,2}=e^{ \pm i \sigma}, \sigma \neq(0, \pi)$, and therefore

$$
\operatorname{Tr} \mathbf{A}\left(\mathbf{x}_{0}^{\mathrm{ell}}\right)=2 \cos \sigma \Rightarrow\left|\operatorname{Tr} \mathbf{A}\left(\mathbf{x}_{0}^{\mathrm{ell}}\right)\right|<2 .
$$

In contrast, $\left|\operatorname{Tr} \mathbf{A}\left(\mathbf{x}_{0}^{\text {hyp }}\right)\right|>2$ for an unstable hyperbolic fixed point [71]. Since these are the only generic types of fixed points, one can consider $\left|\operatorname{Tr} \mathbf{A}\left(\mathbf{x}_{0}\right)\right|<2$ as a criterion for the stability of $\mathbf{x}_{0}$. This yields the condition $-4<-V^{\prime \prime}\left(x_{0}\right)<0$ for the slope of the force.

An accelerator mode at $\left(x_{0}, p_{0}=0\right)$ with velocity $w_{i}=1$ is obtained for $-V^{\prime}\left(x_{0}\right)=1$, which gives a second condition on the force. To obtain a big island around the accelerator mode, the regime within which the linear approximation Eq. (2.33) is valid must be large. 
This can easily be realized with a force that is linear within some range $-V^{(3)}$ (range) $=0$ such that the second derivative of the mapping $\mathbf{f}$ vanishes identically. In order to avoid other islands, the remaining range can be chosen with positive slope of the force, and such that the integral of $V^{\prime}$ over one unit cell vanishes. Fig. 2.11 shows the phase space of such an optimized system with

$$
-V^{\prime}(x)=\left\{\begin{array}{lll}
1-s_{1} x & : & |x| \leq w / 2 \\
s_{2} x-\delta & : & w / 2 \leq|x| \leq 1 / 2
\end{array},\right.
$$

$s_{1}=\frac{1.5}{2 \pi}, s_{2}=50 s_{1}, w=.85$, and $\delta=w /(1-w)$, such that $\int_{-0.5}^{0.5} \mathrm{~d} x-V^{\prime}(x)=0$.
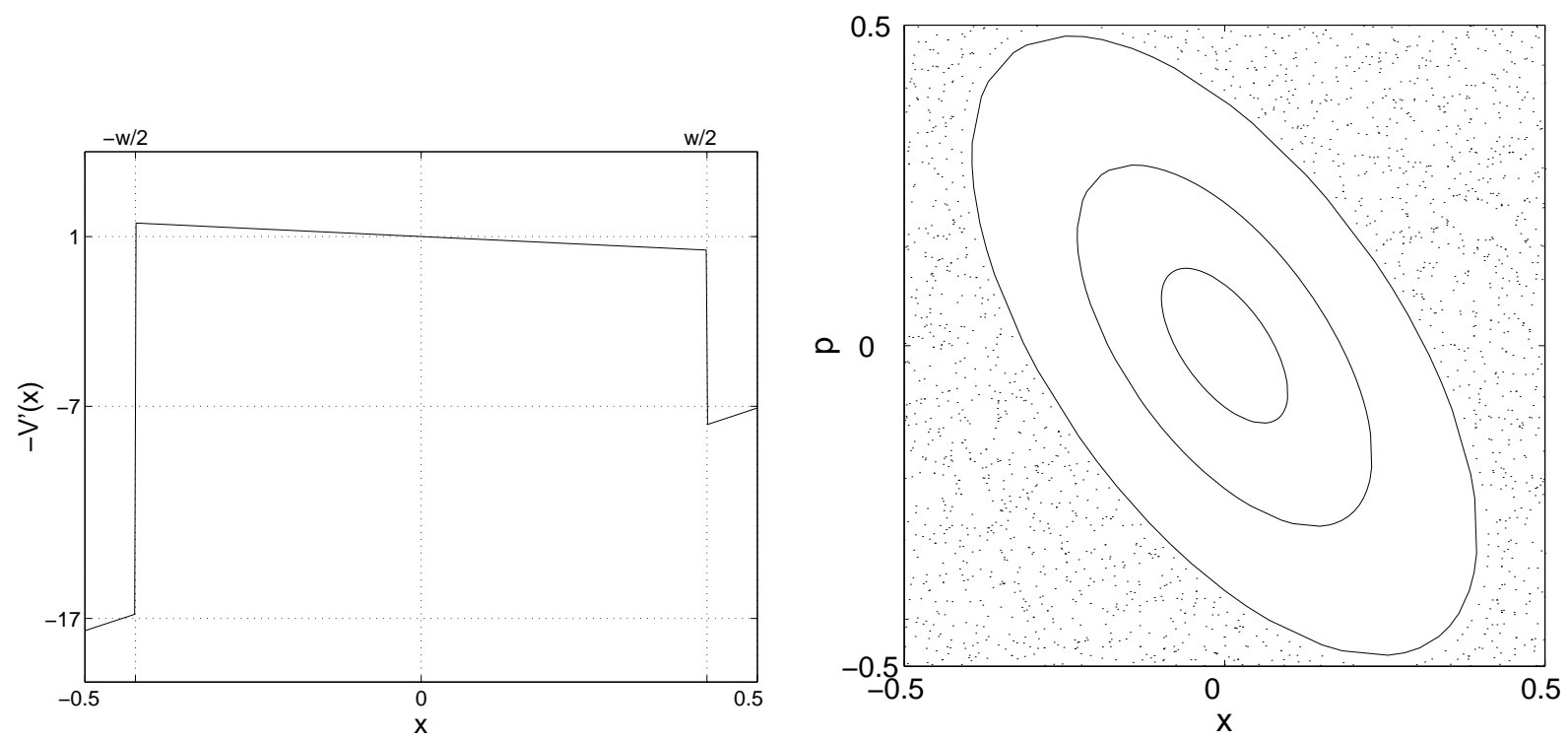

Figure 2.11: (left) Force $-V^{\prime}$ optimized for ballistic transport of the chaotic region according to the method of phase-space engineering. Whereas the section of negative slope supports regular dynamics, the sections of positive slope lead to chaotic motion. (right) Poincaré section of the system with large island transporting at $w_{i}=+1$.

As stated above, quantum mechanics also requires that the potential be smooth, which can be achieved, e.g., by Gaussian smoothing of $V^{\prime}$ : With

$$
G(z)=\exp \left(-z^{2} / 2 \varepsilon^{2}\right) / \sqrt{2 \pi \varepsilon^{2}}
$$

the smoothed functions are calculated as

$$
\tilde{T}^{\prime}(p)=\int d z T^{\prime}(p+z) G(z), \quad \tilde{V}^{\prime}(x)=\int d z V^{\prime}(x+z) G(z) .
$$

One might alternatively Fourier approximate the discontinuous force. The resulting oscillations must be very small, however, and higher orders of the approximation must be taken into account which proves numerically expensive. Finally, $x$ and $p$ must be exchanged. 
Apart from the model given by Eq. (2.27), all the mappings investigated in this thesis have been derived from the standard map and exhibit transporting islands with a size comparable to that of the system Eq. (2.36).

\subsubsection{Sum Rule for Compact Phase Space}

In kicked mappings, the sum rule for transport Eq. (2.14) can take a very simple form. Namely, if the momentum is a periodic variable with period $P$, one can apply the method of reduction to one unit cell also to the $p$-coordinate. Then, the unit cell boundaries take the rôle of the bounding tori, $p_{u}=p_{l}+P=$ const, and since then $T\left(p_{u}\right)=T\left(p_{l}\right)$, transport of the whole unit cell vanishes identically,

$$
A_{\text {ch }} v_{\text {ch }}+A_{\text {reg }} w_{\text {reg }}=0 \text {. }
$$

With $A_{\mathrm{ch}}+A_{\text {reg }}=1, v_{\mathrm{ch}}$ follows immediately from the velocity and area of the regular island as $v_{\mathrm{ch}}=A_{\text {reg }} /\left(1-A_{\text {reg }}\right)$.

For the kicked ratchet Eq. (2.31), Fig. 2.10a, one has $w_{\text {reg }}=-1$, and $A_{\text {reg }}=0.117 \pm 0.001$, thus $v_{\mathrm{ch}}=0.133 \pm 0.001$ in agreement with $v_{\mathrm{ch}}=0.1344 \pm 0.0003$ determined from the spatial distribution shown in Fig. 2.8 b.

\subsection{The Rôle of Lévy Walks}

The sum rule for transport Eq. (2.14), following immediately from the assumption of ergodicity, explains directed chaotic motion. Any knowledge about the details of the dynamics within an invariant set is not required. While explicitly accepting the ergodicity assumption [27] and the sum rule [72], respectively, Flach and colleagues have proposed an alternative explanation for chaotic transport which relies on such details.

Consider the length of a time interval where some chaotic trajectory shows motion in the same direction. In a fully chaotic system, these time intervals are distributed exponentially, $f(t) \sim \mathrm{e}^{-r t}$. In a system with transporting islands, however, the distribution can be approximated with $f(t) \sim t^{-\alpha}$ for $t \rightarrow \infty$ [73]. The long sections of motion in the same direction which lead to this algebraic decay law have been named Lévy walks in contrast to the random walks in a fully chaotic phase space. As Flach et al. claim, the average velocity during the random walks vanishes, whereas the Lévy walks are responsible for the whole transport $[27,72]$. In order to check the consistency of this claim with the sum rule, it is necessary to study the origin of the algebraic law first (for a review, see [35]). 


\subsubsection{The Chain Model for Lévy Walks}

It was outlined in Chapter 2.1.3 that the non-resonant tori are destroyed under increasing perturbation. For a "sufficiently irrational" KAM-torus, this destruction takes place slowly, namely the destroyed torus still acts as a partial barrier for phase-space transport. Trajectories from inside such a cantorus can flow out (and vice versa) only through so-called turnstiles, which correspond to small holes in the cantorus. The flux is proportional to the size of the turnstile [74] and increases with the perturbation [75]. Typically, a chain of cantori surrounds all the regular islands $[76,77]$. To simplifiy the quantitative description it is assumed that

(i) it suffices to take into account only the chain of cantori around the bounding torus of the dominant island (or the bounding extended torus, respectively), neglecting the chains around the small hierarchical islands;

(ii) both the volumes $\Omega_{n}$ enclosed by the $n^{\text {th }}$ and $(n+1)^{\text {th }}$ cantori and the fluxes $\Phi_{n, n+1}$ scale exponentially,

$$
\Omega_{n} \sim \omega^{n}, \quad \Phi_{n, n+1} \sim \phi^{n}, \quad 0<\phi<\omega<1
$$

(iii) the motion inside each volume is stochastic and the probability for a trajectory entering the volume $\Omega_{n}$ to stay inside for a time $t$ is given by

$$
P_{n}(t) \sim \mathrm{e}^{-r_{n} t}, \quad r_{n}=\left\langle t_{n}\right\rangle^{-1}=(\phi / \omega)^{n} .
$$

The resulting model is visualized in Fig. 2.12. Having entered the chain through the first cantorus, a trajectory will explore the chain up to a certain level $n_{\max }$ and finally go back to the chaotic sea. The probability for the trajectory to stay in the chain longer than $t$ can be approximated by an inverse power law,

$$
P(t) \sim t^{-\gamma}, \quad \gamma=\left(1-\frac{\log \omega}{\log \phi}\right)^{-1},
$$

[78], yielding the probability distribution of time intervals

$$
f(t) \sim t^{-\alpha}, \quad \alpha=\frac{\log \omega}{\log \frac{\phi}{\omega}}
$$

For an island with winding number $w_{i}$, staying in the cantori chain implies directed motion at the same velocity, $v_{\text {chain }_{\mathrm{i}}}=w_{i}$. This holds only approximately for an extended torus, $v_{\text {chain }_{\mathrm{a}}} \approx v_{a}$, since the winding number of the destroyed KAM-tori differs from that of the bounding torus. For the purpose of this section, however, this approximation is very good. The chain model can therefore explain the algebraic distribution of time-interval lengths that shows up in typical Hamiltonian ratchets. 


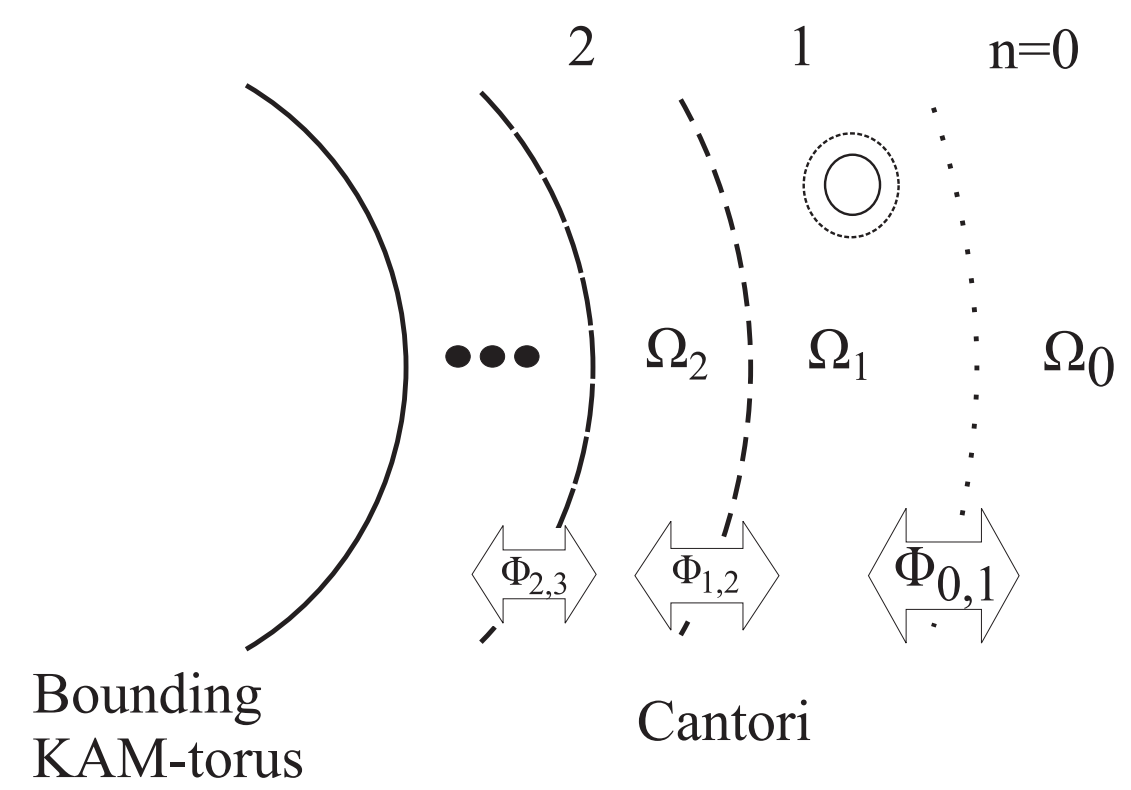

Figure 2.12: Chain model for the sticky region. For simplicity, only the cantori around the major bounding KAM-torus are considered; smaller hierarchical islands as the one shown in the first enclosed volume are neglected. The enclosed volumes and the fluxes decrease exponentially when going to higher levels in the chain.

\subsubsection{Sum Rule for the Reduced Chaotic Set}

In order to explore the asymptotic velocities separately for Lévy walks and random walks, one has to distinguish them by some well-defined method. This distinction is not straightforward. Denisov and Flach [72] approach the problem numerically. Running a chaotic trajectory for long times, they calculate the change in position $\Delta x_{j}=x_{j}-x_{j-1}$ at integer times $t=t_{0}+j$. A change in the sign of $\Delta x_{j}$ marks the end of the corresponding walk. This yields the distribution of walk lengths $f\left(t_{\text {walk }}\right)$. For small $t_{\text {walk }}$, the distribution shows exponential decay ascribed to the random walks, in contrast to the power law decay for large $t_{\text {walk. }}$ A cutoff time is chosen as $t_{\text {cut }}=10 T$, where $T$ denotes the time period of the system. A walk with length smaller than $t_{\text {cut }}$ is consequently considered as a random walk and the opposite as a Lévy walk. In the chain model, this can be related to a decomposition of the chaotic set (see Fig. 2.13). For simplification, it is assumed that the length of a certain walk is essentially given by the inverse of the flux rate of the innermost cantorus $N_{\max }$ reached in this walk ( $N$ counts the cantori, while $n$ denotes the intermediate volumes). This is fulfilled if $r_{N-1} \gg r_{N}$, i.e. if the flux rates decay very fast. Then, a cutoff cantorus $N_{\text {cut }}$ can be determined from $r_{N_{\text {cut }}}<1 / t_{\text {cut }}$ AND $r_{N_{\text {cut }}-1}>1 / t_{\text {cut }}$. For each regular island and each extended KAM-torus, such a cutoff cantorus separates a chain region from the reduced chaotic set. In a typical ratchet phase space, the asymptotic velocity of the reduced 


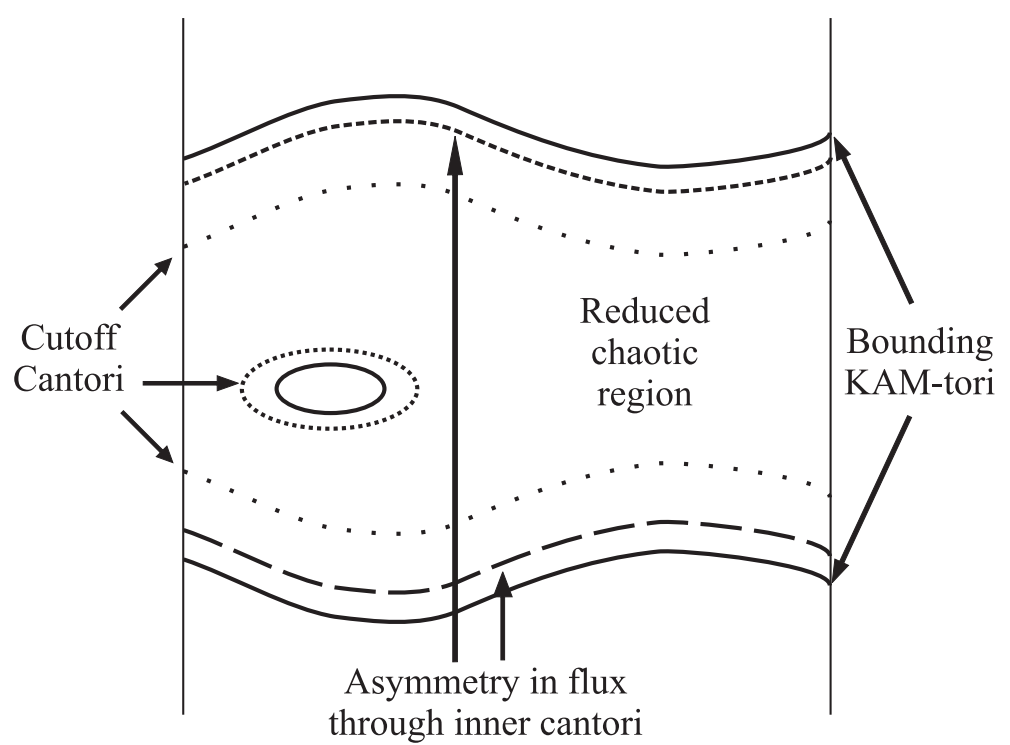

Figure 2.13: Decomposition of the chaotic sea for one unit cell. The upper and lower cutoff cantori are symmetric while the higher levels of the corresponding chains is sketched asymmetrically. For a closed KAM-torus, the cutoff leads to an enlarged effective volume.

chaotic set can be calculated in analogy to Eq. (2.17) as

$$
v_{\text {red }}=\frac{\langle T\rangle_{u}-\langle T\rangle_{l}-\sum_{i} A_{i} w_{i}}{A_{\text {red }}}
$$

with $u$ and $l$ now denoting the upper and lower cutoff cantori and $A_{i}$ the area (or volume, respectively) enclosed by the cutoff cantorus of island $i$.

There is no reason to assume that this velocity must vanish. Rather, it is shown in Fig. 2.14 that this is not the case for the variant of the kicked Harper map. With a nonsmoothed force Eq. (2.27), the positive chaotic velocity $v_{\mathrm{ch}}=0.121$ read out from a chaotic trajectory after $10^{6}$ time periods is well in agreement with the value $v_{\mathrm{ch}}=0.121$ calculated from $A_{\text {reg }}=0.108$ according to the sum rule (all the errors are estimated as \pm 0.001 , cf. Ch. 2.2.4). In terms of the length distributions of walks in positive or negative direction, $f_{+}\left(t_{\text {walk }}\right)$ and $f_{-}\left(t_{\text {walk }}\right)$, the positive value for the chaotic velocity is due to $f_{+}>f_{-}$for small $t_{\text {walk }}$ (Fig. 2.14(left)). According to the observation that the distribution functions decay exponentially, Lévy walks are not visible. This can be explained with the instantaneous increase of the perturbation parameter at $(x+t) \bmod 1=0.15,0.85$ which destroys the cantori chain in the vicinity of the transporting island. For a Gaussian smoothed force (cf. Ch 2.3.2, $\varepsilon=0.1$ ), running a trajectory yields $v_{\mathrm{ch}}=0.101$, which is compared to $v_{\text {ch }}=0.103$ from the sum rule $\left(A_{\text {reg }}=0.093\right)$. In this model, the smoothed force allows the existence of a cantori chain around the island, which transports in negative direction. Consequently, Lévy walks are visible in the distribution function $f_{-}$(Fig. 2.14(right)). Calculating $v_{\mathrm{ch}}$ from these long walks according to the method outlined in [72] would therefore yield a 
completely wrong result. In this model system, Lévy flights are neither required for the onset of directed chaotic motion nor can one calculate the chaotic velocity by analyzing them.
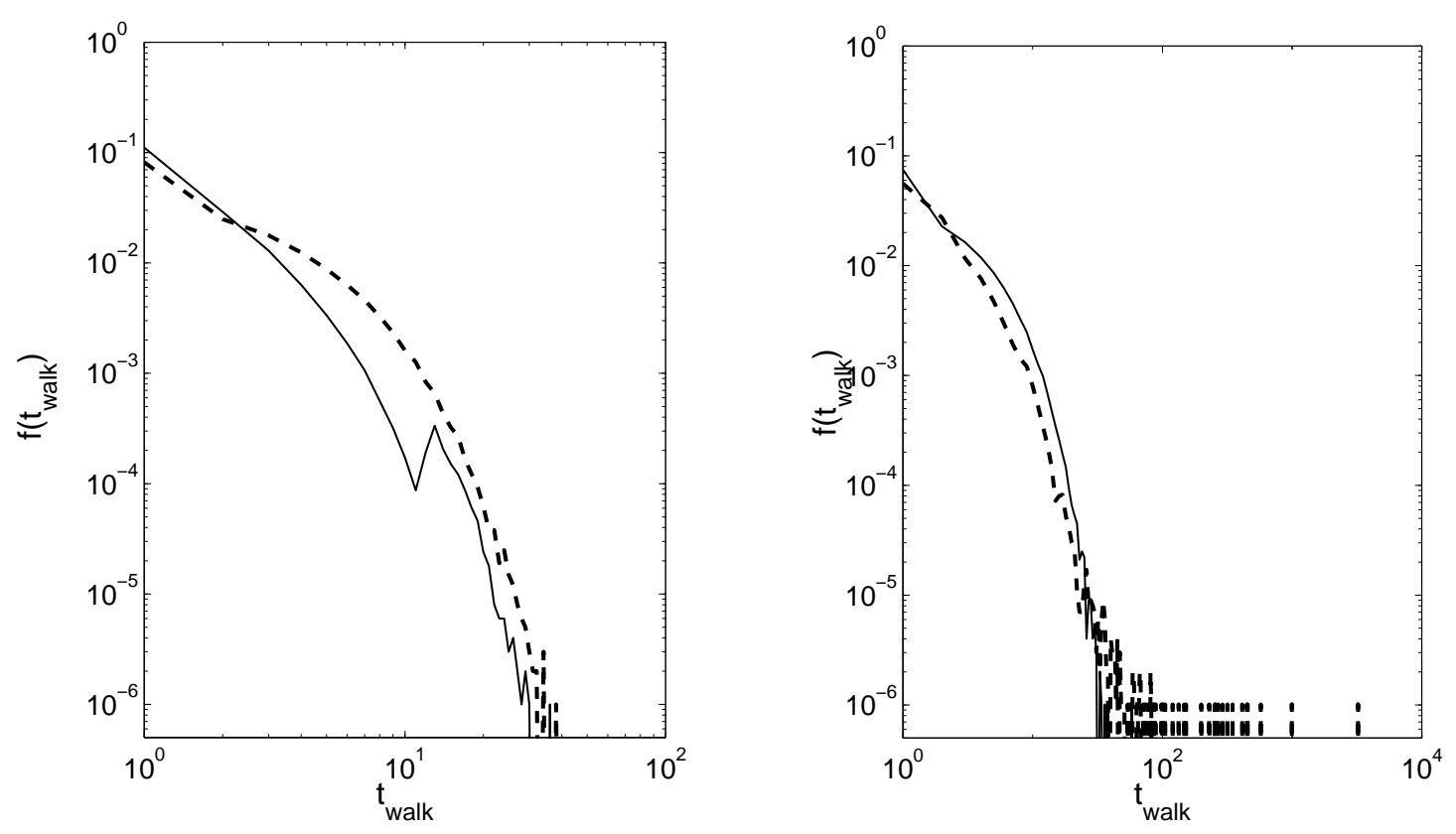

Figure 2.14: Length distributions $f_{ \pm}\left(t_{w a l k}\right)$ of walks in positive (solid curve), and negative (dashed curve) direction, respectively. The distributions are calulated from a chaotic trajectory iterated over $10^{6}$ time steps. (left) Distributions for the model specified by Eq. (2.27). (right) Same for a corresponding system with a smoothed force.

However, Denisov and Flach specify a model where cutting off the long walks with $t_{f}>10 \mathrm{~T}$ indeed yields a vanishing average velocity for the remaining steps of the trajectory [72]. The Hamiltonian is given by $H(x, p, t)=p^{2} / 2+V(x, t)$ with

$$
V(x, t)=-\cos (x)-x\left(E_{1} \cos (t)+E_{2} \cos (2 t+\Phi)\right),
$$

with $E_{1}=0.252, E_{2}=0.052$, and $\Phi \neq 0, \pi$ [72]. If $E_{2}=0$, the system had time-reversal symmetry and $v_{\mathrm{ch}}=v_{\text {red }}=0$. This symmetry is broken only weakly since $E_{1} \approx 5 E_{2}$. The walk length distribution exhibits an asymmetry for $t_{f}>40 T$, leading to $v_{\text {ch }} \neq 0$ while $v_{\text {red }} \approx 0$. In the chain model, this can be explained with an asymmetry between the higher levels of the upper and lower cantori chains while the lower levels - and in particular the cutoff tori - are still symmetric (Fig. 2.13). For a stronger breaking of the symmetry, $E_{1} \approx 3 E_{2}$, the reduced chaotic set is no more symmetric and $v_{\text {red }} \neq 0$ [72].

Not only can the restriction to Lévy walks yield completely wrong results for the average velocity of the chaotic set, it also increases the numerical effort as stated in Ch. 2.2.4. Concluding, it is important to note that chaotic transport can arise from purely chaotic motion. 


\subsection{Outlook}

This chapter is concerned with the description of one-dimensional dissipationless ratchets. As stated above, already a two-dimensional system with periodic driving would not fulfill a central requirement, namely the existence of impenetrable barriers for transport in a mixed phase space. Here, a chaotic trajectory can diffuse from "inside" a regular torus to the outside such that almost all points in phase space belong to the same chaotic set. It might therefore be of interest to study the long-time behavior of such systems.

Up to now, the optimization of phase space has been carried out for kicked maps. The construction of a model with a potential which is continuous in time might could bring the theory closer to applications. Finally, the study of Hamiltonian systems is perfectly suited for the switch to quantum mechanics which will be performed in the next chapter. 


\section{Chapter 3}

\section{Perfect Quantum Hamiltonian Ratchets}

This chapter deals with the quantum versions of Hamiltonian ratchets. The name perfect quantum Hamiltonian ratchet is used to distinguish the periodic case from that with disorder. A perfect quantum ratchet is characterized by the fact that spatial periodicity is preserved by the quantization (cf. Ch. 3.1.2). Here, one observes a paradox: On the one hand, the system shows quantum tunneling between the classically invariant phase-space regions, i.e. these regions are not invariant any more. This violates a fundamental prerequisite for classical transport (Fig. 3.1(left)). On the other hand, the velocity expectation value of a wave packet does not vanish even in the long-time limit, see (Fig. 3.1(right)), that is to say, quantum transport persists.

The first section introduces the basics as required for the following studies. In the second section, the paradox is explained by a quantum analogue to the classical sum rule for transport which is employed for the analysis of wavepacket transport in the long-time limit. The quantum sum rule being based on the spectrum of eigenvalues of the timeevolution operator, one can also extract information about the short-time dynamics from this spectrum, as carried out in the third section. Finally, the Chern index, a topological property of the spectrum which was discussed in connection with transport is investigated for perfect quantum ratchets. 

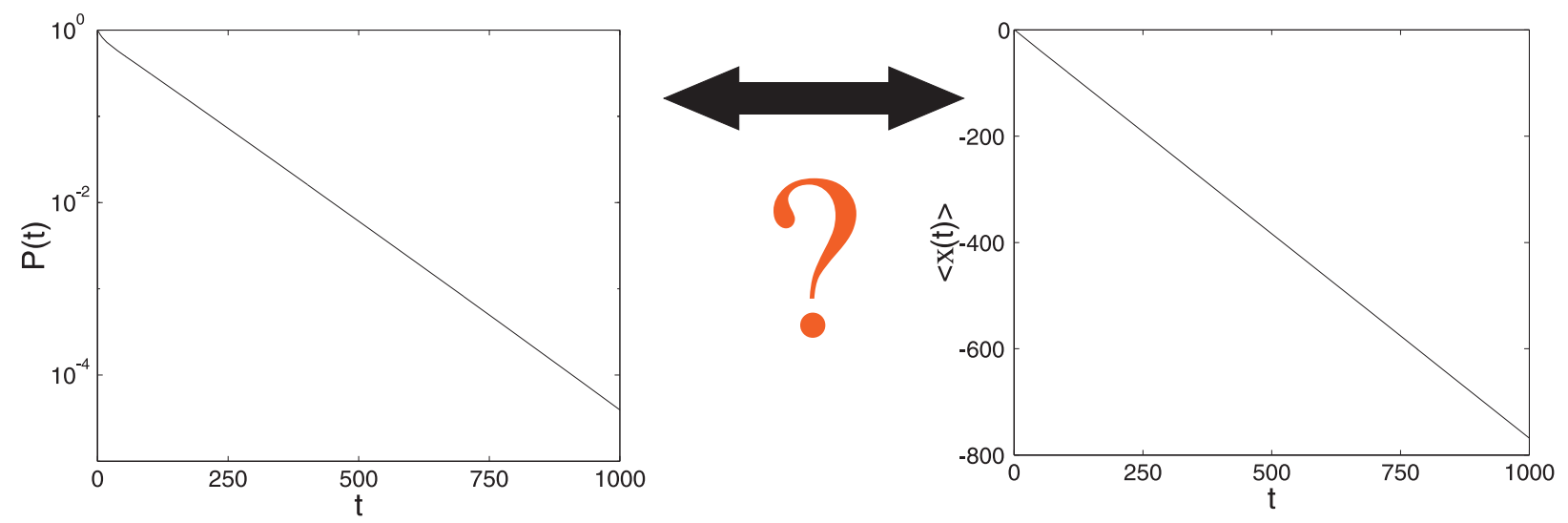

Figure 3.1: (left) Quantum tunneling from the transporting island with classical transport velocity $v_{\text {class }}=-1$. For a coherent wave packet centered initially on the elliptic fixed point the weight inside the island decays exponentially with time. The chosen model is the quantum version of the ratchet described by Eq. (2.31) at $h=1 / 20$. (right) Quantum transport for the same wave packet. The initial quantum transport velocity is preserved.

\subsection{Quantization of Hamiltonian Ratchets}

The Schrödinger equation for a general Hamiltonian ratchet in position representation reads

$$
i \hbar \frac{\partial}{\partial t} \psi(x, t)=\hat{H}(x, \hat{p}, t) \psi(x, t)=(\hat{T}(\hat{p})+V(x, t)) \psi(x, t),
$$

with $V(x, t)=V(x, t+1)=V(x+X, t)$ (later, the spatial period will be set to $X=1$ ). For kicked quantum maps, the classical versions of which were introduced in Ch. 2.3, the calculation of the eigenvalues and eigenfunctions of $H$ is simplified drastically due to the delta-like time dependence. Therefore, almost all the quantum calculations in this thesis are carried out for such models. In the first subsections, the implications of Bloch's theorem and its analogon for time-periodic systems, th Floquet theorem, are studied. Then, the technique of visualizing the quantum phase space is described, followed by a statement about the quantum manifestations of a mixed classical phase space.

\subsubsection{Periodicity in Time}

In analogy to Bloch's theorem for spatially periodic systems, an eigenstate $\psi_{\alpha}(x, t)$ of a time-periodic Hamiltonian, $\hat{H}(t)=\hat{H}(t+1)$, can be written as a product of a steady state $u_{\alpha}(x, t)=u_{\alpha}(x, t+1)$ and an exponential which depends on time, $\psi_{\alpha}(x, t)=\mathrm{e}^{-i \varepsilon_{\alpha} t / \hbar} u(x, t)$ [79]. Since $\psi_{\alpha}$ solves the Schrödinger equation (3.1), the steady state satisfies

$$
\left[\hat{H}(t)-i \hbar \frac{\partial}{\partial t}\right] u_{\alpha}(x, t)=\varepsilon_{\alpha} u_{\alpha}(x, t) .
$$


Considering the operator in the square brackets as a new Hamiltonian, with the time treated as an ordinary coordinate with periodic boundary conditions. $\varepsilon$ takes the place of an energy, the reason for which it is called quasienergy. It is convenient to rescale the quasienergy as $\epsilon_{\alpha}=\varepsilon_{\alpha} / 2 \pi \hbar$. Then, adding integers to $\epsilon$ does not change the eigenstate and one can set $\epsilon \in[0,1)$.

Since

$$
\psi_{\alpha}(x, t+1)=\mathrm{e}^{-2 \pi i \epsilon_{\alpha}(t+1)} u_{\alpha}(x, t+1)=\mathrm{e}^{-2 \pi i \epsilon_{\alpha}} \psi_{\alpha}(x, t),
$$

diagonalizing the time-evolution operator over one period $\hat{U}\left(t_{0}+1, t_{0}\right)$ yields the quasienergies of the system. This is especially important since for a kicked map, $\hat{U}$ takes a simple multiplicative form,

$$
\hat{U}\left(t_{0}+1, t_{0}\right)=\hat{U}\left(t_{0}+1, t_{0}+1-\delta\right) \hat{U}\left(t_{0}+1-\delta, t_{0}\right)=\mathrm{e}^{-i \hat{V}(\hat{x}) / \hbar} \mathrm{e}^{-i \hat{T}(\hat{p}) / \hbar},
$$

with $t_{0}+1$ just after and $t_{0}+1-\delta$ just before the kicking. The special form of this unitary Floquet operator allows an efficient numerical implementation of the time evolution: One can change sequentially from position to momentum representation by a Fourier transform, leaving the kick, and the free time evolution respectively, as a mere multiplication of the diagonal representation of the Floquet operator and the vector which represents the wave packet. With Hilbert-space dimension $N$, the calculation time for the Fourier transformation scales only as $N \log N$, in contrast to the scaling with $N^{2}$ of the multiplication with the non-diagonal Floquet operator matrix.

\subsubsection{Periodicity in Space and Momentum}

According to Bloch's theorem, spatial periodicity in one dimension implies that $\psi(x, t)=$ $\mathrm{e}^{2 \pi i k x / X} u(x, t)$, where $u(x+X, t)=u(x)$ is now a spatially periodic function and the rescaled Bloch phase $k \in[0,1)$ is also referred to as quasimomentum. The momentum representation is calculated by a standard Fourier transform,

$$
\tilde{\psi}(p, t)=\int_{-\infty}^{+\infty} \mathrm{d} x \mathrm{e}^{-i p x / \hbar} \mathrm{e}^{2 \pi i k x / X} u(x, t)
$$

Decomposing the spatial coordinate into a small value denoting position within a unit cell and an integer labeling the unit cells, $x=\xi+n X, \xi \in[0, X)$, and $n \in \mathbb{N}$, yields

$$
\begin{aligned}
\tilde{\psi}(p, t) & =\int_{0}^{X} \mathrm{~d} \xi \mathrm{e}^{i \xi(-p / \hbar+2 \pi k / X)} u(\xi, t) \sum_{n=-\infty}^{+\infty} \mathrm{e}^{i n X(-p / \hbar+2 \pi k / X)} \\
& =a_{k} \delta((-p / \hbar+2 \pi k / X) \bmod 2 \pi) .
\end{aligned}
$$

That is to say, one obtains a discrete momentum basis $|m\rangle$ with $\hat{p}|m\rangle=\frac{2 \pi \hbar}{X}(m+k)|m\rangle=$ $\frac{h}{X}(m+k)|m\rangle$, where $m$ is an integer. For a given Bloch phase, the interval between two 
momentum lattice sites is given by $\Delta p=h / X$. Now, the kicked Hamiltonians studied in Ch. 2.3 also exhibit a periodic kinetic energy, i.e. $T(p)=T(p+P)$ in momentum representation, which in analogy yields a discrete position basis with $\Delta x=h / P$. Then, $\Delta x \Delta p=h^{2} / X P$. One can measure position and momentum in units of the corresponding unit cells in order to obtain dimensionless variables. Then, $\Delta x / X=\Delta p / P=h / X P=$ $h_{\text {eff }}$, i.e. Planck's constant should be scaled by the area of the stroboscopic unit cell to obtain a dimensionless quantity. The semiclassical limit is then simply given by $h_{\text {eff }} \rightarrow 0$. Henceforth, $X=P=1$, such that there is no need to introduce additional notations for scaled variables. We will use $h$ whenever a statement exploits the periodicities, while $\hbar$ will be used in expressions of more general validity.

The discreteness of the position basis, however, influences the applicability of Bloch's theorem: only if $1 / h$ is an integer, the discrete basis fits into the period of the potential and the time-evolution operator is exactly periodic after one unit cell. In the case that $1 / h=l / m$ is rational, periodicity is still preserved for a unit-cell chain of length $m$. With an irrational $h$, however, quantum periodicity is violated. The studies of this situation are postponed to the next Chapter. Here, for simplicity, $1 / h \in \mathbb{N}$. One can then restrict the extended system to one unit cell and consider the Bloch phase as a parameter of the eigenstates. Since $k$ determines the phase which is multiplied to the wavefunction upon crossing the cell's boundary, a variation of $k$ amounts to a variation of the boundary conditions of the spatial unit cell.

The $k$-dependence can also be assigned to the momentum operator. With the unitary transformation $R(x)=\mathrm{e}^{2 \pi i k x}$, the momentum operator for the restricted system is given by $\hat{p}(k)=R^{\dagger} \hat{p} R=\hat{p}+h k$ : on the one hand,

$$
\psi_{k}^{*} \hat{p} \psi_{k}=u^{*} R^{\dagger} \hat{p} R u,
$$

and on the other hand,

$$
\begin{aligned}
\hat{p}(k) u(x, t) & =\left[-i \hbar \frac{\partial}{\partial x}+h k\right]\left(\mathrm{e}^{-2 \pi i k x} \psi_{k}(x, t)\right) \\
& =-h k u(x, t)+\mathrm{e}^{-2 \pi i k x}\left[-i \hbar \frac{\partial}{\partial x}\right] \psi_{k}(x, t)+h k u(x, t) \\
& =R^{\dagger}(x) \hat{p} \psi(x, t),
\end{aligned}
$$

such that $\psi_{k}^{*} \hat{p} \psi_{k}=u^{*} \hat{p}(k) u$. The Bloch-phase dependence of the momentum operator transfers to the Hamiltonian, $\hat{H}(k)=\hat{H}(x, \hat{p}(k), t)$ (see App. A.2), such that the eigenvalues of $\hat{H}(k)$ become functions of $k$, referred to as the Floquet bands $\epsilon_{\alpha}=\epsilon_{\alpha}(k)$.

With both spatial and temporal periodicity, the Hilbert space can be reduced to the spatiotemporal unit cell, $(\xi, t) \in[0,1)$, with an inner product given by

$$
\langle\langle\cdot\rangle\rangle=\int_{0}^{1} \mathrm{~d} \xi \int_{0}^{1} \mathrm{~d} t(\cdot) .
$$




\section{Avoided Crossings}

For a classically nonintegrable system without symmetries, the Floquet bands typically do not cross each other. For the eigenenergies of a Hamiltonian which change under variation of some parameter, this was explained in [80]. The generalization to Floquet operators can be found in [81] Ch. 3.2. For two non-degenerate bands, one considers the two-dimensional Hilbert space spanned by the corresponding eigenstates. Since the Floquet operator of this reduced Hilbert space is again unitary, it depends on four real parameters,

$$
U_{\mathrm{red}}=\left(\begin{array}{cc}
c_{1}+i c_{2} & c_{3}+i c_{4} \\
-c_{3}+i c_{4} & c_{1}-i c_{2}
\end{array}\right)
$$

with the additional restriction that $\sum c_{i}^{2}=1$. Therefore, there are three free parameters in the equation $e_{ \pm}=c_{1} \pm i \sqrt{c_{2}^{2}+c_{3}^{2}+c_{4}^{2}}$ for the eigenvalues of $U_{\text {red }}$. To achieve that $e_{+}=e_{-}$, one must generically vary three parameters in the Floquet operator. This number is reduced to two if $U_{\text {red }}$ symmetric such that $c_{3} \equiv 0$. However, under variation of only one parameter, e.g., the Bloch phase $k$, the bands generically avoid crossings.

\subsubsection{Quantum Phase-Space Representations}

In the classical case, the phase space of a system can be visualized by plotting the precise location of the section of a trajectory with the Poincare surface. A stroboscopic quantum phase-space representation, however, faces the problem that position and momentum obey Heisenberg's uncertainty principle. Wigner $[64,82]$ exploited the statistical interpretation of quantum mechanics to arrive at an analogue to the Boltzmann distribution function for the probability of some particle to be at the point $(x, p)$ in phase space. Such a function $\Psi$ should satisfy three conditions. First, the integral over position (momentum) space should yield the absolute square of the momentum (position) representation of the state $\phi$,

$$
\int \mathrm{dx} \Psi(\mathrm{x}, \mathrm{p})=|\phi(\mathrm{p})|^{2}, \quad \int \mathrm{dp} \Psi(\mathrm{x}, \mathrm{p})=|\phi(\mathrm{x})|^{2} .
$$

Second, this function should be real and, third, positive, according to the definition of probability. While the Wigner function

$$
\Psi(x, p)=\frac{1}{\sqrt{2 \pi \hbar}} \int \mathrm{dx}^{\prime} \mathrm{e}^{-\frac{\mathrm{i}}{\hbar} \mathrm{px}^{\prime}} \phi^{*}\left(\mathrm{x}-\mathrm{x}^{\prime} / 2\right) \phi\left(\mathrm{x}+\mathrm{x}^{\prime} / 2\right)
$$

satisfies the first two conditions, it fails for the third one due to the oscillations induced by the Fourier transform. In the semiclassical limit, though, it can be shown that the this function becomes positive definite and approaches the classical probability distribution.

In order to get rid of the oscillations, one can smooth the Wigner function with a Gaussian both in position and momentum. The resulting Husimi distribution function, introduced 
in [83], reads

$$
\Phi\left(x_{0}, p_{0}\right)=\frac{1}{\pi \hbar} \int \mathrm{d} x^{\prime} \mathrm{d} p^{\prime} \exp \left[-\frac{\left(x_{0}-x^{\prime}\right)^{2}}{\hbar \omega^{2}}-\omega^{2} \frac{\left(p_{0}-p^{\prime}\right)^{2}}{\hbar}\right] \Psi\left(x^{\prime}, p^{\prime}\right)
$$

where $\omega$ is the width of the position uncertainty [64]. One can now choose an overcomplete basis of Gaussians centered around different $\left(x_{0}, p_{0}\right)$ to obtain a stroboscopic phase-space representation of $\phi$. This representation will be used throughout the thesis.

\subsubsection{Regular and Chaotic States}

It is one of the fundamental hypotheses in quantum physics of the mixed phase space that the stationary states in the semiclassical limit are confined to the classically invariant regions of phase space $[40,42]$. In Ch. 4 , an important and unexpected exemption from this hypothesis will be introduced for extended systems. In this chapter, however, dealing with exactly periodic systems, which can be restricted to a compact phase space, it can rightfully be assumed. Intuitively, in order to observe a state confined to an invariant set, $\Delta x \Delta p \sim h$ should be smaller than the phase-space area of this set. According to the observations, this is indeed sufficient (Fig. 3.2). Then, the eigenstates of a mixed system can be categorized into chaotic or regular states. With a smaller $h$, also the fine structure of phase space is resolved and the regular states will condense on the classical tori, such that there will be one eigenstate at the island's center while the other eigenstates supported by this island arrange as fringes around this center. Decreasing $h$ even further, Ketzmerick and colleagues [47] showed that even the hierarchical regions can support their own eigenstates. For the purpose of this thesis, these hierarchical states can be considered as a subclass of the regular ones. With $h$ small enough to permit an appropriate statistics, the fraction of regular and chaotic states equals the fractions of the areas of the corresponding classical regions in phase space.

\subsection{Reading Transport from the Slopes of Floquet Bands}

This section contains the main results for perfect quantum Hamiltonian ratchets: First, the velocity expectation value of a steady state is shown to be encoded in the corresponding Floquet band. That relation leads directly to a quantum analogue to the sum rule for compact phase space. Shifting from eigenstates to wavepackets, the coexistence of quantum tunneling with non-vanishing asymptotic transport is explained. 

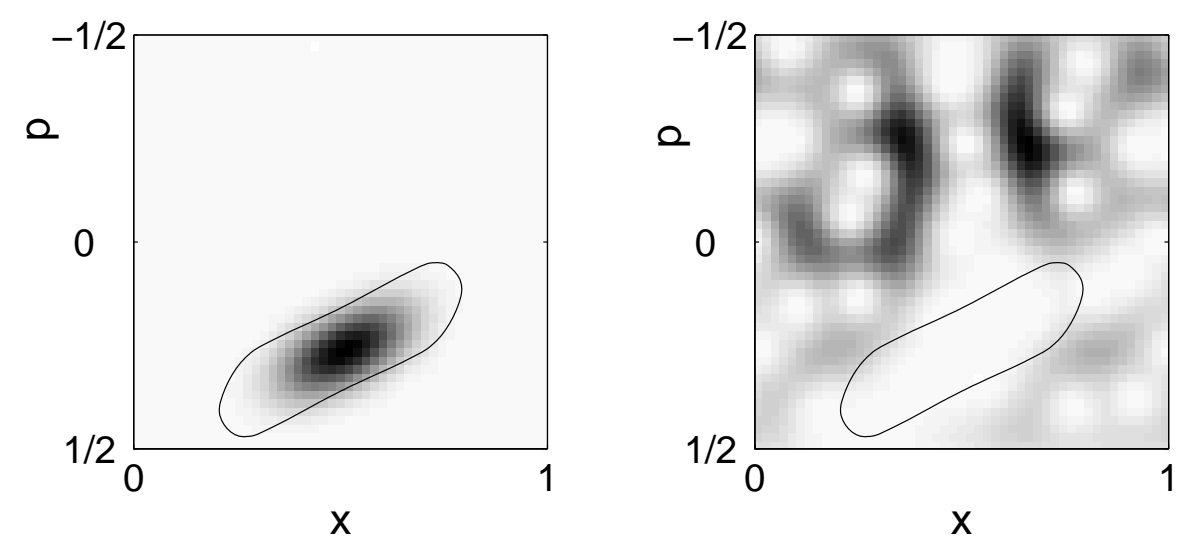

Figure 3.2: Husimi representations of a regular (left) and a chaotic (right) eigenstate. The light areas correspond to small values of the Husimi function, and vice versa. For comparison, the bounding torus of the classical regular island is plotted (solid line).

\subsubsection{The Quantum Sum Rule for Transport}

Classically, the transport of a finite invariant phase-space component equals the averaged velocity weighted with the component's volume (see Eq. (2.13)). In analogy, quantum transport of a classically invariant phase-space set is given by the sum over the velocity expectation values of those eigenstates $\psi_{\alpha, k}(x, t)$ which are supported by this set. With the velocity operator $\hat{v}=\hat{T}^{\prime}(\hat{p})$, the expectation value of an eigenstate reads

$$
v_{\alpha, k} \equiv\left\langle\left\langle\psi_{\alpha, k}|\hat{v}| \psi_{\alpha, k}\right\rangle\right\rangle \text {. }
$$

In App. A, it is shown that this equals the slope of the Floquet band $\alpha$ at the position $k$, namely

$$
v_{\alpha, k}=\left\langle\left\langle\psi_{\alpha, k}\left|\hat{T}^{\prime}(\hat{p})\right| \psi_{\alpha, k}\right\rangle\right\rangle=\mathrm{d} \epsilon_{\alpha}(k) / \mathrm{d} k .
$$

This allows one to study quantum transport in terms of spectral properties of the system, namely in terms of the slopes of Floquet bands.

Consider the bands shown in Fig. 3.3. On a coarse scale, the straight bands with negative slope appear to cross the oscillating ones with positive average slope. Plotting the phasespace representation of an eigenstate corresponding to a quasienergy on a straight band shows that this eigenstate is a regular one (see Fig. 3.2(left)). Accordingly, quasienergies on the fluctuating bands correspond to chaotic states (see Fig. 3.2(right)). The magnification in the inset shows that the bands actually do not cross, as argued in Ch. 3.1.2. Only in the semiclassical limit, the difference in quasienergies at an avoided crossing becomes exponentially small. One can then imagine switching from the adiabatic viewpoint with avoided crossings to a diabatic one with real crossings, [84,85]. While in the actual, the adiabatic case, the states corresponding to the two bands of an avoided crossing exchange their type, 


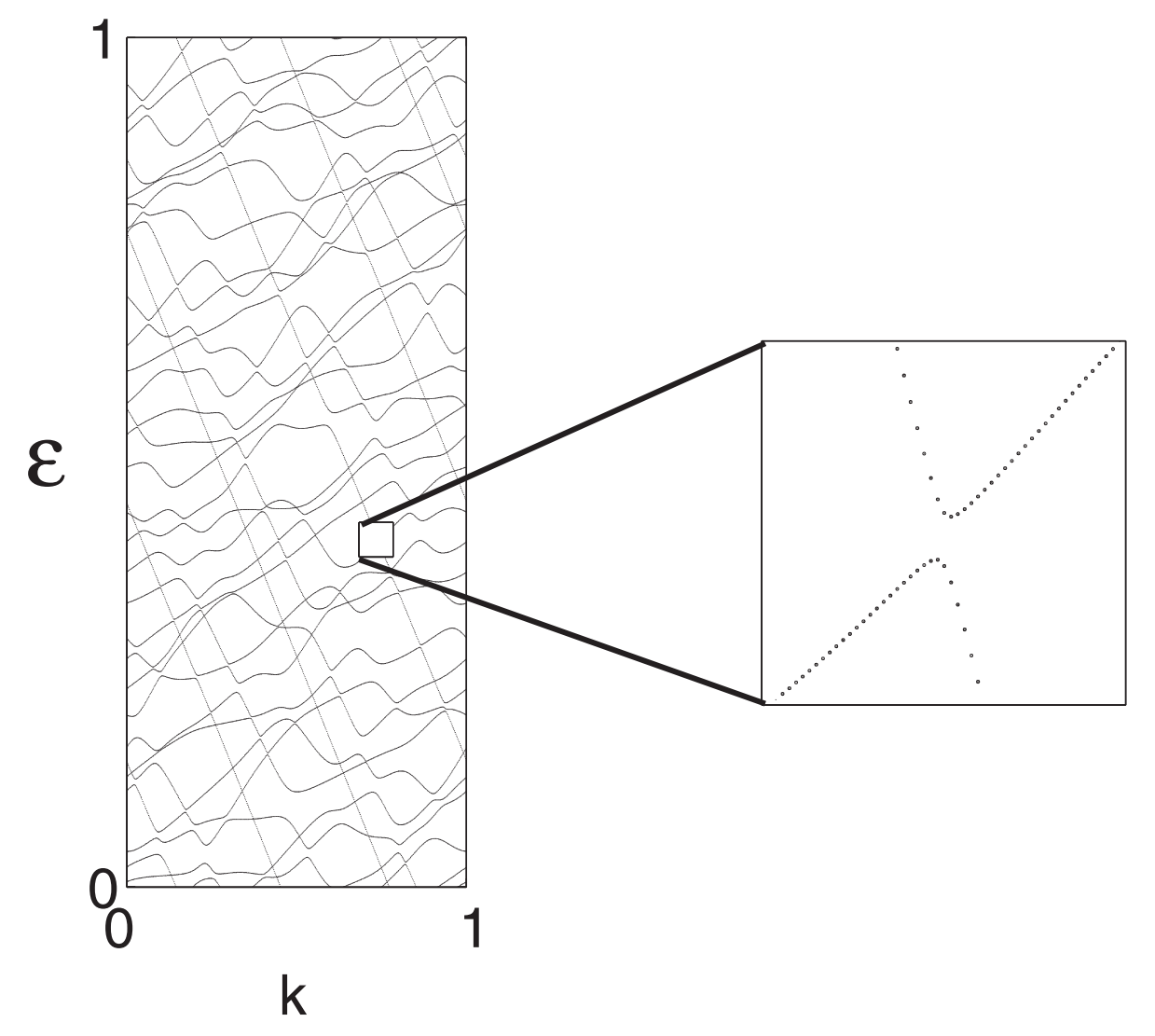

Figure 3.3: Floquet bands $\epsilon(k)$ of the quantized mapping Eq. 2.31 for $h=1 / 128$. Quasienergies corresponding to regular states lead to straight lines with negative integer slope in contrast to the oscillating chaotic bands. On a sufficiently fine scale, all crossings are avoided (right inset).

one can uniquely characterize the bands as regular or chaotic in the diabatic picture. At an avoided crossing, one observes eigenstates with a "mixed" Husimi representation, supported by both classical phase-space sets which are involved in the avoided crossing. The fraction of these special $k$-sections vanishes in the semiclassical limit and does therefore not violate the hypothesis that the eigenstates are confined to the classical sets in this limit. The generalized Hellman-Feynman theorem, Eq. (3.13), explains the band structure visualized in Fig. 3.3: Neglecting the avoided crossings, a regular band exhibits a slope equivalent to the velocity of the classically invariant regular set. For the model system given by Eq. (2.31), one has regular bands with $\omega^{(\mathrm{reg})}=w_{i}=-1$. Alternatively, if $\psi_{\alpha, k}$ for a given $k$ is a chaotic state, it spreads over the chaotic sea with the weight oscillating under variation of $k$, causing the corresponding band slope to oscillate as well.

Now, the overall slope of the bands can be described in terms of their winding number around the torus $(\epsilon, k) \in[0,1)$. In the adiabatic picture, each band closes on itself, i.e. all the winding numbers are zero. In the diabatic picture, however, the bands must close only after an integer number of periods in the $\epsilon$ and $k$ directions, such that the winding numbers 
$\omega_{\alpha, \text { diab. }} \equiv \omega_{\alpha}$ are rational. Since shifting from the adiabatic to the diabatic viewpoint simply corresponds to a reconnection of the bands at the crossings, the sum of winding numbers must be preserved. Thus it must be zero also in the diabatic case, $\sum_{\alpha} \omega_{\alpha}=0$. Restricting the analysis to one regular and one chaotic component, the sum can be decomposed into the contributions from regular and chaotic bands,

$$
\sum_{\alpha} \omega_{\alpha}^{(\mathrm{reg})}+\sum_{\alpha} \omega_{\alpha}^{(\mathrm{ch})}=0 .
$$

This is the quantum-mechanical analogue of the classical sum rule (2.38). Because of the localization of the regular states on tori inside the regular island, the winding number of the regular bands in $(\epsilon, k)$-space is in the semiclassical limit identical to the winding number in $(x, t)$-space of the central periodic orbit, i.e., $\omega_{\alpha}^{(\mathrm{reg})}=w_{\text {reg. }}$. Moreover, in this limit, the fractions of regular and chaotic bands correspond to the stroboscopic phase-space areas $A_{\text {reg }}$ and $1-A_{\text {reg }}$, respectively. We therefore obtain from Eq. (3.14) the mean slope of the chaotic bands, $\bar{\omega}^{(\mathrm{ch})}=A_{\text {reg }} /\left(1-A_{\text {reg }}\right)=v_{\mathrm{ch}}$, as the classical drift velocity. This is confirmed in Fig. 3.4.

\subsubsection{Asymptotic Wavepacket Transport}

Up to now, only the (averaged) velocity expectation values of stationary states have been considered. The band slopes, however, can also be used to calculate the velocity expectation value of an arbitrary wave packet $\phi(x, t)$ in the long-time limit. $x$ denotes position in the extended chain of unit cells, $x \in(-\infty,+\infty)$, which is again decomposed as $x=\xi+n$ into the position within a unit cell $\xi \in[0,1)$, and the unit-cell index $n$. Accordingly, the position expectation value of $\phi(x, t)$ can be split into two parts, namely one which corresponds to length scales within a unit cell and a coarse one corresponding to the scale of many unit cells,

$$
\begin{aligned}
x(t) & =\int_{-\infty}^{+\infty} \mathrm{d} x x|\phi(x, t)|^{2}=\int_{0}^{1} \mathrm{~d} \xi \sum_{n=-\infty}^{+\infty}(\xi+n)|\phi(\xi+n, t)|^{2} \\
& =x_{\mathrm{uc}}(t)+x_{\mathrm{tr}}(t) .
\end{aligned}
$$

The contribution from the dynamics inside the unit cells is bounded by the size of the unit cell,

$$
x_{\mathrm{uc}}(t)=\int_{0}^{1} \mathrm{~d} \xi \xi \sum_{n=-\infty}^{+\infty}|\phi(\xi+n, t)|^{2} \leq \int_{0}^{1} \mathrm{~d} \xi \sum_{n=-\infty}^{+\infty}|\phi(\xi+n, t)|^{2}=1,
$$

as follows from the normalization of $\phi$. For the investigation of directed transport, therefore, only the coarse part is relevant,

$$
x_{\operatorname{tr}}(t)=\int_{0}^{1} \mathrm{~d} \xi \sum_{n=-\infty}^{+\infty} n|\phi(\xi+n, t)|^{2} .
$$




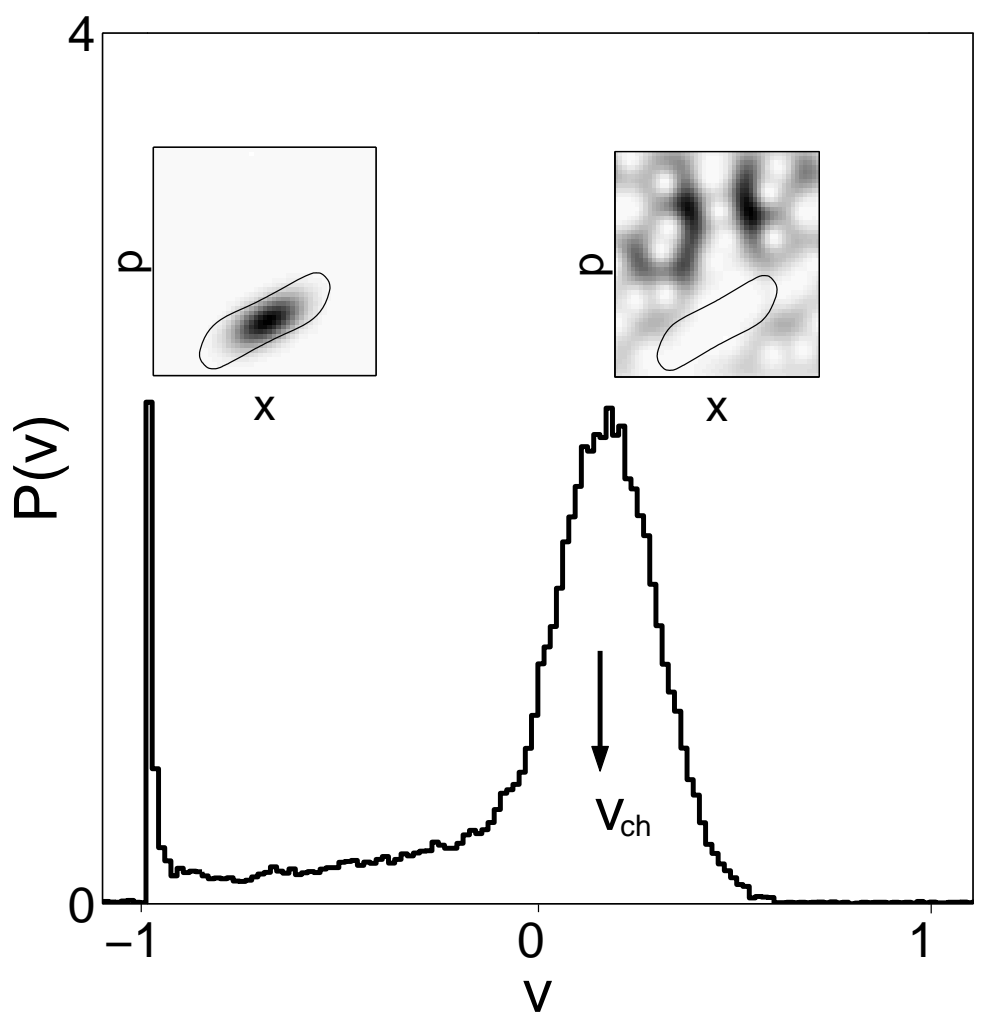

Figure 3.4: Distribution of the slopes of Floquet bands shown in Fig. 3.3 exhibiting a sharp peak at $v=-1$ from the regular bands and a Gaussian distribution with maximum close to $v_{\mathrm{ch}}$ from the chaotic ones, together with the Husimi representations of typical eigenstates. The arrow indicates the classical drift velocity $v_{\mathrm{ch}}$ of the chaotic component. The weight in between the sharp peak and the Gaussian is due to the intermediate slopes at the avoided crossings and will therefore vanish for $h \rightarrow 0$.

In App. B, it is shown that

$$
x_{\operatorname{tr}}(t) \approx v_{\infty} t, \quad v_{\infty}=\int_{0}^{1} \mathrm{~d} k \sum_{\alpha}\left|c_{\alpha, k}\right|^{2} v_{\alpha, k},
$$

where $c_{\alpha, k}$ is the overlap of $\phi$ with the eigenstate $\psi_{\alpha, k}$ at time $t=0$ and $v_{\alpha, k}=\frac{\mathrm{d} \epsilon_{\alpha}(k)}{\mathrm{d} k}$ is the velocity expectation value of $\psi_{\alpha, k}$.

In other words, the average velocity expectation value of some wave packet is the average over all band slopes weighted with the overlaps with the Floquet states. Considering wavepackets which are initially localized in a single unit cell and, moreover, inside one of the invariant sets of the classical dynamics, the weights $\left|c_{\alpha, k}(0)\right|^{2}$ are concentrated on the (diabatic) bands corresponding to this invariant set, but they are nearly homogeneously distributed over the whole Brillouin zone $k \in[0,1)$ since localization in position implies delocalization in (quasi-) momentum. This is illustrated in Fig. 3.5. Consequently, from 
Eq. (3.18), one obtains for the asymptotic velocity of a wavepacket which is initially well localized inside an invariant set of the classical phase an average over the band slopes of the (adiabatic) bands associated with this invariant set which in the previous section was shown to coincide with the average classical velocity of the corresponding invariant set.
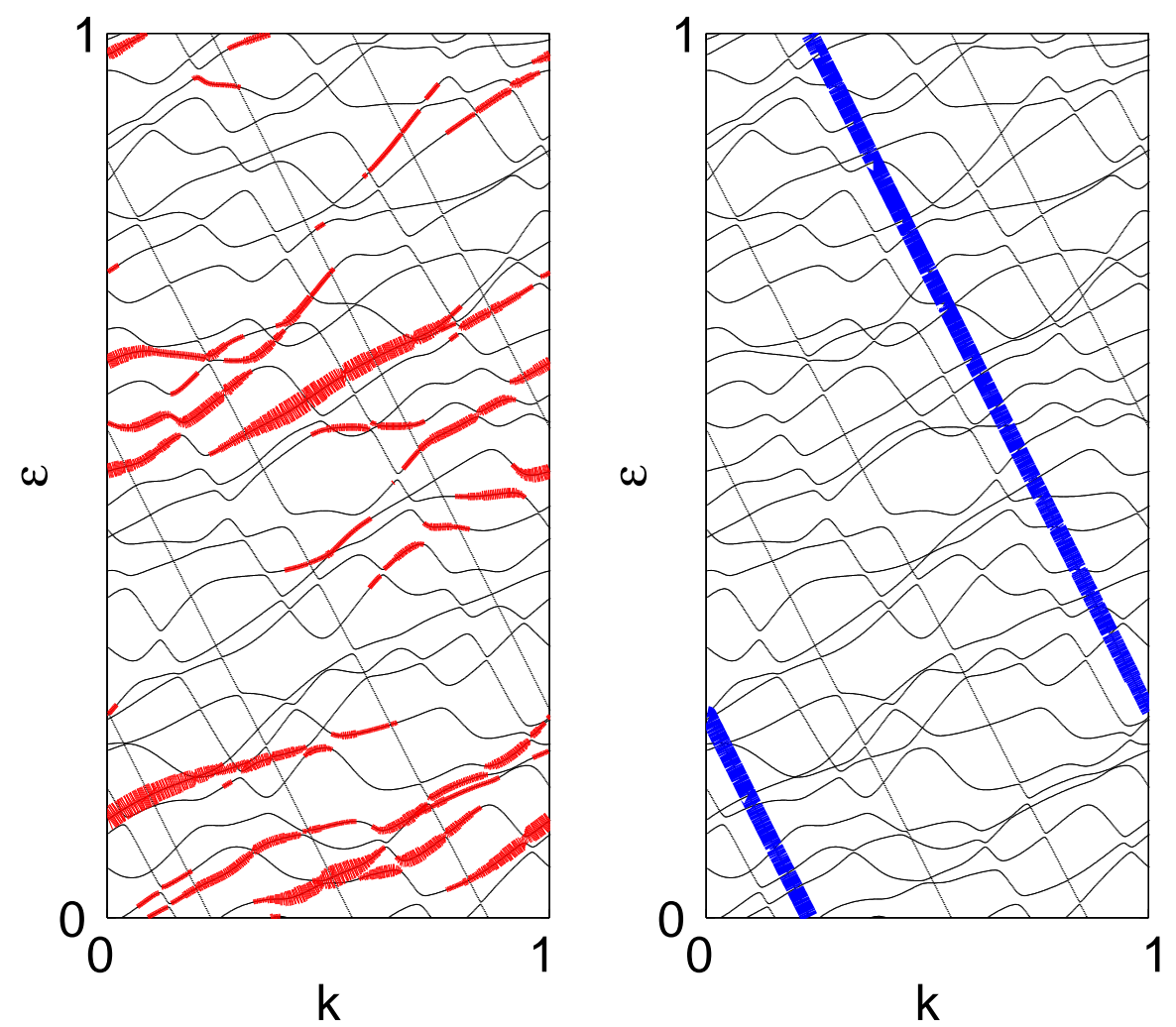

Figure 3.5: Quasienergy band spectrum of system Eq. (2.31) for $h^{-1}=32$. The linewidth encodes the overlap $\left|\left\langle\phi_{\alpha, k} \mid \psi\right\rangle\right|^{2}$ of the corresponding eigenstate $\left|\phi_{\alpha, k}\right\rangle$ of the time-evolution operator with an initial wavepacket $|\psi\rangle$. (left) The wavepacket is a coherent state located in the chaotic part of the phase space of a single unit cell. (right) Here, the wavepacket is concentrated on one of the tori inside the regular island of a unit cell.

This conclusion is valid only in the semiclassical regime, since otherwise the notion of diabatic bands, pertaining to a certain classically invariant set, breaks down. On the other hand, in the semiclassical regime it is clear from the outset that for short times the velocity of a wavepacket corresponds to the classical velocity. Nevertheless, the statement for asymptotic transport is non-trivial since the classical velocity shows up in the asymptotic dynamics for long time, i. e. beyond the $h$-dependent time scale for quantum-classical correspondence. The result is illustrated in Fig. 3.6, where the average position of chaotic wavepackets is shown over a large time interval. In agreement with Eq. (3.18) one observes a linear dependence on time with very small fluctuations, i. e. directed ballistic quantum transport persists in the long-time limit. The value of the velocity depends on the initial 
conditions but these fluctuations decrease with $h$ such that the average approaches the value of the classical velocity. In contrast, in the deep quantum regime one can strongly vary the asymptotic velocity by choosing different initial positions of the wave packet.

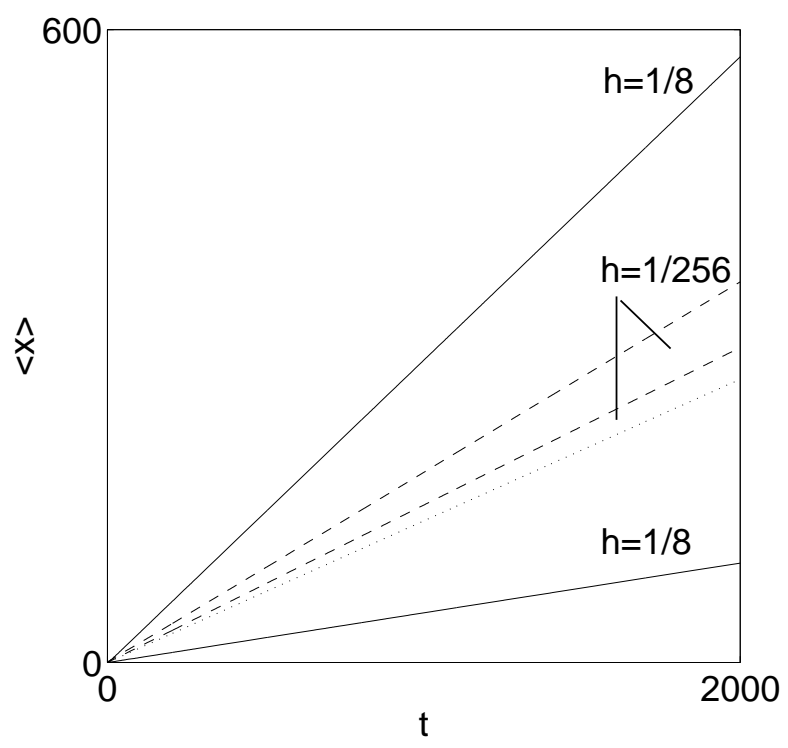

Figure 3.6: Position vs. time for wavepackets initialized as coherent states inside the chaotic part of phase space of system Eq. 2.31. Two different values of $h$ and two different initial conditions are used. The dotted line shows the classical chaotic transport velocity.

It should be noted that the typical quantum velocity for a chaotic wavepacket is above the classical value. This is due to the hierarchical phase-space region around an island which communicates with the main chaotic sea only via leaky cantori. Depending on $h$ some of these cantori allow quantum transitions only by tunneling and therefore are effectively part of the regular island [47]. According to the sum rule, a larger island means that the chaotic transport velocity must be larger.

\subsubsection{Dynamical Tunneling}

As stated above, it is surprising that the division of classical phase space into invariant sets influences the long-time quantum dynamics, because classically impenetrable barriers can be crossed in quantum dynamics by tunneling. Indeed, this phenomenon, which is best known for the case of energy barriers (e. g. in a double-well potential), has been generalized to dynamical barriers and was recently confirmed experimentally [51]. If in quantum dynamics no strict barriers exist, the wave packet should explore for long times the whole available phase space forcing the directed transport to vanish. It was shown in the previous section that this is not the case. So how does tunneling show up in Hamiltonian ratchets? 


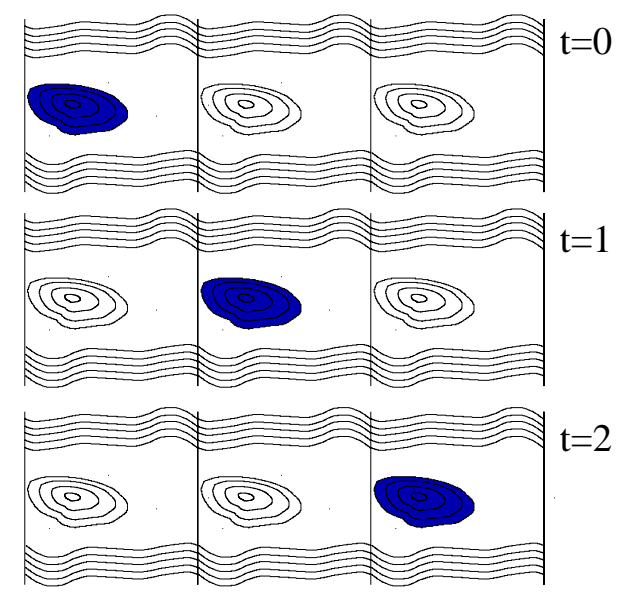

Figure 3.7: A classical distribution prepared in the regular island of one unit cell moves on the chain without any spreading. The speed corresponds to the rational winding number of the central periodic orbit of the island $\left(\omega_{\mathrm{r}}=1\right.$ in this case).

To answer this question one considers a wavepacket which is initially prepared inside the regular island in phase space in unit cell $n=0$, e. g. the wave packet shown in the left inset of Fig. 3.4. Classically, such an initial distribution is simply shifted along the chain with a velocity corresponding to the winding number $w_{i}$ of the central periodic orbit (Fig. 3.7). This property is conserved in the quantum dynamics if one neglects the narrow avoided crossings between the adiabatic regular and chaotic bands: In this case the regular bands are straight lines

$$
\epsilon_{\alpha, k}=\epsilon_{\alpha, 0}+w_{i} k
$$

Within this approximation, a localized wavepacket initially constructed as a superposition of all states of a regular band $r$ which pertains to the island $i,|\phi(x, t=0)\rangle=\int_{0}^{1} \mathrm{~d} k\left|\psi_{\mathrm{r}, k}(x)\right\rangle$, is transported at speed $w_{i}$ without any spreading,

$$
\begin{aligned}
\left|\phi\left(\xi+w_{i} t, t\right)\right\rangle & =\int_{0}^{1} \mathrm{~d} k \mathrm{e}^{-2 \pi \mathrm{i} \epsilon_{\mathrm{r}, k} t}\left|\psi_{\mathrm{r}, k}\left(\xi+w_{i} t\right)\right\rangle \\
& =\int_{0}^{1} \mathrm{~d} k \mathrm{e}^{2 \pi \mathrm{i}\left(k w_{i}-\epsilon_{\mathrm{r}, k}\right) t}\left|\psi_{\mathrm{r}, k}(\xi)\right\rangle \\
& =\phi(\xi, 0),
\end{aligned}
$$

where $t$ is assumed to be a multiple of the period of the regular periodic orbit.

Since excluding avoided crossings between regular and chaotic bands from the analysis obviously prohibits quantum tunneling out of the island, it follows in turn that this tunneling is encoded in the avoided crossings. These show up in the regular bands as deviations from the straight line $\epsilon=w_{i} k$ : since the bands are bent towards the chaotic bands, the actual slope is $k$-dependent and slightly smaller than $w_{i}$. Using this qualitative information allows 
to predict the shape of the wavepacket at very large times as

$$
\begin{aligned}
|\phi(\xi+n, t)\rangle & =\int_{0}^{1} \mathrm{~d} k \mathrm{e}^{2 \pi \mathrm{i}\left(k n-\epsilon_{\mathrm{r}, k} t\right)} \psi_{\mathrm{r}, k}(\xi) \\
& =\sum_{r} \sum_{k: \epsilon_{\mathrm{r}, k}^{\prime}=\frac{n}{t}} \sqrt{\frac{i}{\left|\epsilon_{\mathrm{r}, k}^{\prime \prime} t\right|}} \mathrm{e}^{2 \pi \mathrm{i}\left(k \epsilon_{\mathrm{r}, k}^{\prime}-\epsilon_{\mathrm{r}, k}\right) t} \psi_{\mathrm{r}, k}(\xi),
\end{aligned}
$$

where $\psi_{\mathrm{r}, k}(\xi)$ is treated as a slowly varying prefactor of the rapidly oscillating phase. The points of stationary phase in $k$ in Eq. (3.20) select those Bloch states whose superposition yields the wavepacket at time $t$ and position $n$. It is no surprise that these are exactly those points for which the slope of the band corresponds to the velocity $n / t$. Due to avoided crossings, the actual slope of the regular bands is smaller than $w_{i}$, and hence for the transition to the unit cell $n=w_{i}$, where all classical probability is concentrated, no points of stationary phase with real $k$ exist such that to leading order this process is forbidden in quantum mechanics. There might be complex solutions of the equation $\epsilon_{\mathrm{r}, k}^{\prime}=w_{i}$, but there the exponent in Eq. (3.20) has a real part and the contribution will be exponentially small in $t$ (cf. Figs. 3.1 (left), 3.8). Indeed, the well-known law for the weight in the unit cell $n=w_{i} t$ for disordered transporting systems [86], $P(t)=\left|\phi\left(w_{i} t, t\right)\right|^{2}=\mathrm{e}^{-\gamma t}$ with tunneling rate $\gamma$, also applies to perfect Hamiltonian ratchets (cf Ch. 4.5.2).

The main part of the wavepacket, however, is concentrated not in the "classical" unit cell but rather at positions for which real points of stationary phase exist in Eq. (3.20). These correspond to velocities which have a narrow distribution around a value which is slightly smaller than the classical velocity. Due to this dispersion in the velocities, induced by avoided crossings, the wavepacket will spread ballistically in time and will be concentrated right behind the classical position (Fig. 3.8).

For a wavepacket initially prepared in the chaotic part of a unit cell the influence of tunneling is much less pronounced: Although the narrow avoided crossings with regular bands do modify the chaotic bands as well, the existence of points of stationary phase in an expansion similar to Eq. (3.20) is unaffected. Due to the wide avoided crossings between them, the chaotic states by themselves exhibit a large variation in their velocities around the classical value.

The rôle of tunneling in Hamiltonian ratchets is now identified. It corresponds to the avoided crossings between regular and chaotic states (or between regular states with different winding numbers). In the dynamics of initially localized wavepackets tunneling shows up mainly in the evolution of regular states which slightly lag behind the position expected from classical considerations. Quantum tunneling is not able to hinder directed ballistic transport of such wavepackets for asymptotically large times.

For systems with a symmetric classical phase space like the standard map this implies that in the semiclassical regime and for initially localized wavepackets there will be no significant dynamical tunneling from an island to its symmetry-related partner (or the chaotic sea): 


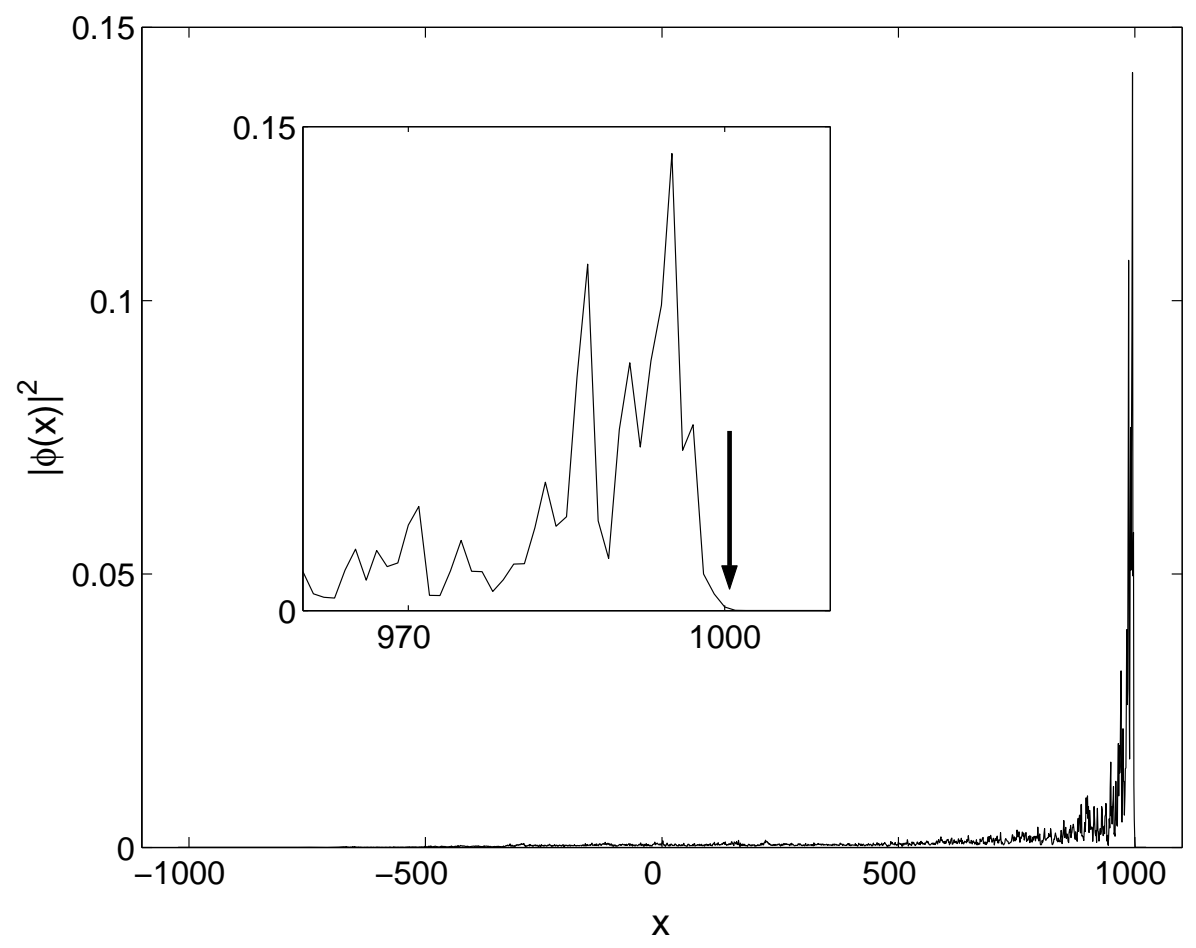

Figure 3.8: The same wave packet as in Fig 3.1 in position representation at time $t=1000$. The classical probability would be restricted to the unit cell $n=1000$ (arrow in the inset). The quantum wave packet, however, has spread and the peak of the weight distribution lags slightly behind due to tunneling. This is in accordance with the exponential decay of $P(t)$ shown in Fig. 3.1 (left).

A wavepacket initialized in a single unit cell and well inside an "accelerator mode" with positive velocity will never be caught moving backwards. There is no contradiction of this statement with the experiments reported in [51], because there delocalized initial states were prepared for which directed transport in the sense of Hamiltonian ratchets is meaningless. With respect to the band spectrum, such states can be concentrated right on an avoided crossing. This will dramatically enhance the effect of dynamical tunneling.

\subsection{Short-Time Transport and the Generalized Form Factor}

The analysis based on the winding numbers carried out in the previous section can be applied to predict the mean quantum transport in the semiclassical regime from the classical value. Even more information, namely that about the time course of quantum transport, can be extracted from spectral two-point correlation functions, as will be seen in the 
following.

For short times, the quantum dynamics is expected to follow the classical one, since quantum interference can be neglected [81] Ch. 8. A suitable quantity to establish a direct relation between the time evolution of the classical distribution and the band spectrum is the generalized form factor introduced and studied in [39] for completely chaotic systems. It is defined as a double Fourier transform of the spectrum, from energy to time and from quasimomentum to unit-cell index. With $t \in \mathbb{N}$,

$$
K_{n}(t)=h\left\langle\left|a_{n}(t)\right|^{2}\right\rangle
$$

where

$$
a_{n}(t)=\int_{0}^{1} \mathrm{~d} k \mathrm{e}^{+2 \pi \mathrm{i} k n} \operatorname{tr} \hat{U}_{k}^{t}=\sum_{\alpha=1}^{N} \int_{0}^{1} \mathrm{~d} k \mathrm{e}^{+2 \pi \mathrm{i}\left(k n-\epsilon_{\alpha, k} t\right)} .
$$

The average denoted by $\langle\ldots\rangle$ in Eq. (3.21) can be taken over a small time interval or over an ensemble of quantum systems corresponding to approximately the same classical system. Comparing Eq. (3.22) to Eq. (3.20) and neglecting all details of the dynamics within unit cells which are contained in $\psi_{\alpha, k}(\xi)$, one can see that $a_{n}(t)$ can be interpreted as a wavepacket after time $t$ which is formed by a homogeneous superposition from the whole band spectrum such that it is localized initially within the unit cell $n_{0}=0$.

To confirm this interpretation, it is shown in App. C, that the form factor $K_{n}(t)$ is related semiclassically to the classical dynamics of an initially homogeneous distribution in phase space. It is assumed that upon the averaging, the regular and the chaotic bands vary independently such that the form factor can be written as an incoherent sum $K_{n}(t)=K_{n}^{(\mathrm{reg})}(t)+K_{n}^{(\mathrm{ch})}(t)$, as in [43]. The quality of this approximation improves in the semiclassical limit, when most avoided crossings between the chaotic and the regular subspectra become very narrow. According to Ch. 3.2, the $f_{i} / h$ bands pertaining to the regular island $i$ are straight lines with slope $\mathrm{d} \epsilon / \mathrm{d} k=w_{i}$. At fixed Bloch number $k$, the distribution of the corresponding quasienergies is Poissonian in the semiclassical limit. Using these facts in (3.21) yields

$$
K_{n}^{(\mathrm{reg})}(t)=P_{n}^{(\mathrm{reg})}(t)=\sum_{i} f_{i} \delta_{n-w_{i} t}
$$

where $P_{n}^{(\text {reg) }}(t)$ is the classical time-dependent unit-cell distribution for the regular phasespace component (cf. App. C.1).

A semiclassical theory for the generalized form factor in a system with a fully chaotic dynamics was presented in $[39,87]$. In order to apply it to a system with a mixed classical phase space it is assumed that the classical periodic orbits fill the chaotic component homogeneously as in a fully chaotic system [88]. In this case, the result of $[39,87]$ remains essentially unchanged, $K_{n}^{(\mathrm{ch})}(t)=\tau P_{n}^{(\mathrm{ch})}(t)$, where $\tau=t f_{\mathrm{ch}} / h$ is the rescaled time for 
systems with broken time-reversal symmetry (see App. C.2). Combining the two results, one arrives at

$$
K_{n}(t)=P_{n}^{(\mathrm{reg})}(t)+\tau P_{n}^{(\mathrm{ch})}(t) .
$$

Therefore, in the semiclassical limit, the generalized form factor can be expressed in terms of classical distribution functions only. Since in the derivation of (3.23), correlations between
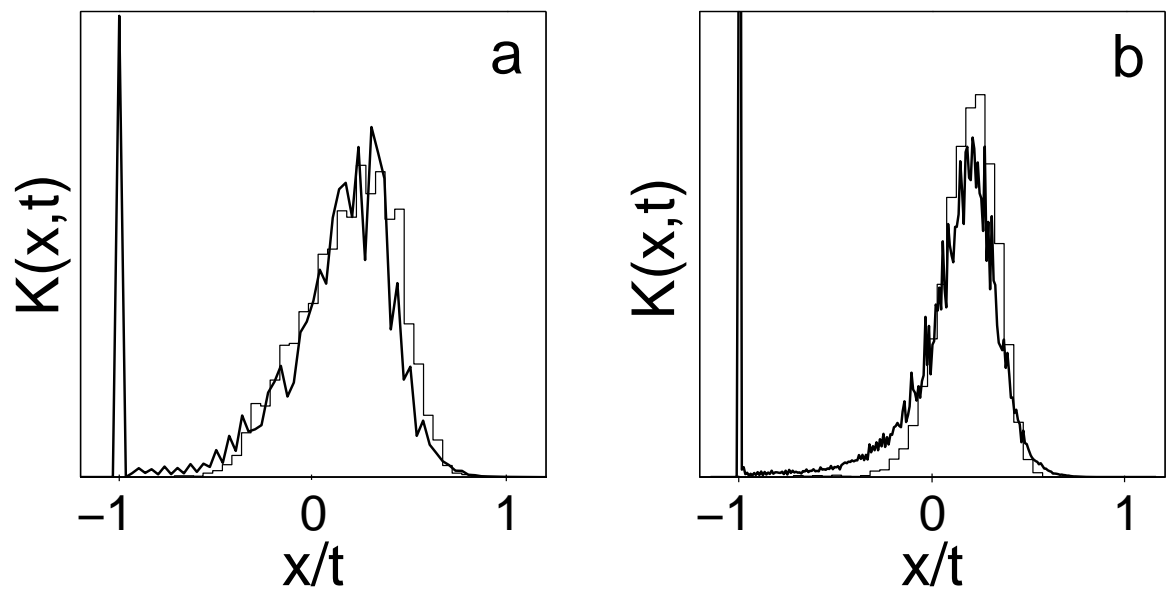

Figure 3.9: Form factor $K_{n}(t)$ for the system Eq. 2.31 at $h=1 / 128$ (thick line) at times $t=30$ (a), and $t=111$ (b), the Heisenberg time of the chaotic component. The two distinct peaks centered around $w_{i}$ and $v_{\mathrm{ch}}$ are the signature of ballistic transport. The comparison of the time-dependent width of the chaotic distribution with the classical distribution $P_{n}^{\mathrm{ch}}(t)$ of chaotic trajectories (thin line) supports Eq. 3.23.

different classical orbits have been neglected, it is valid only up to the Heisenberg time $t_{H}^{\mathrm{ch}}=f_{\mathrm{ch}} / h$ of the chaotic component [89]. For this time, Fig. 3.9 confirms the validity of Eq. 3.23. Together with the above analysis for the long-time limit, this result rounds out the investigation of perfect quantum Hamiltonian ratchets.

\subsection{Chern Indices for Perfect Ratchets}

Chern indices have been used to determine the conduction properties of mixed systems [90,91]. Thouless et al. [92], by referring to the fact that these numbers must be integers, could establish the connection to the quantized Hall conductance which intensified the interest in the topic. Leboeuf et al. [93,94] use them in the distiction of the eigenstates of the kicked Harper model into topologically localized and extended. In the following, the theory is applied to Hamiltonian ratchets.

If the system of interest is periodic also in the momentum variable, $T(p+P)=T(p)$, a formalism analogous to the one which for periodicity in $x$ yields the quasimomenta will 


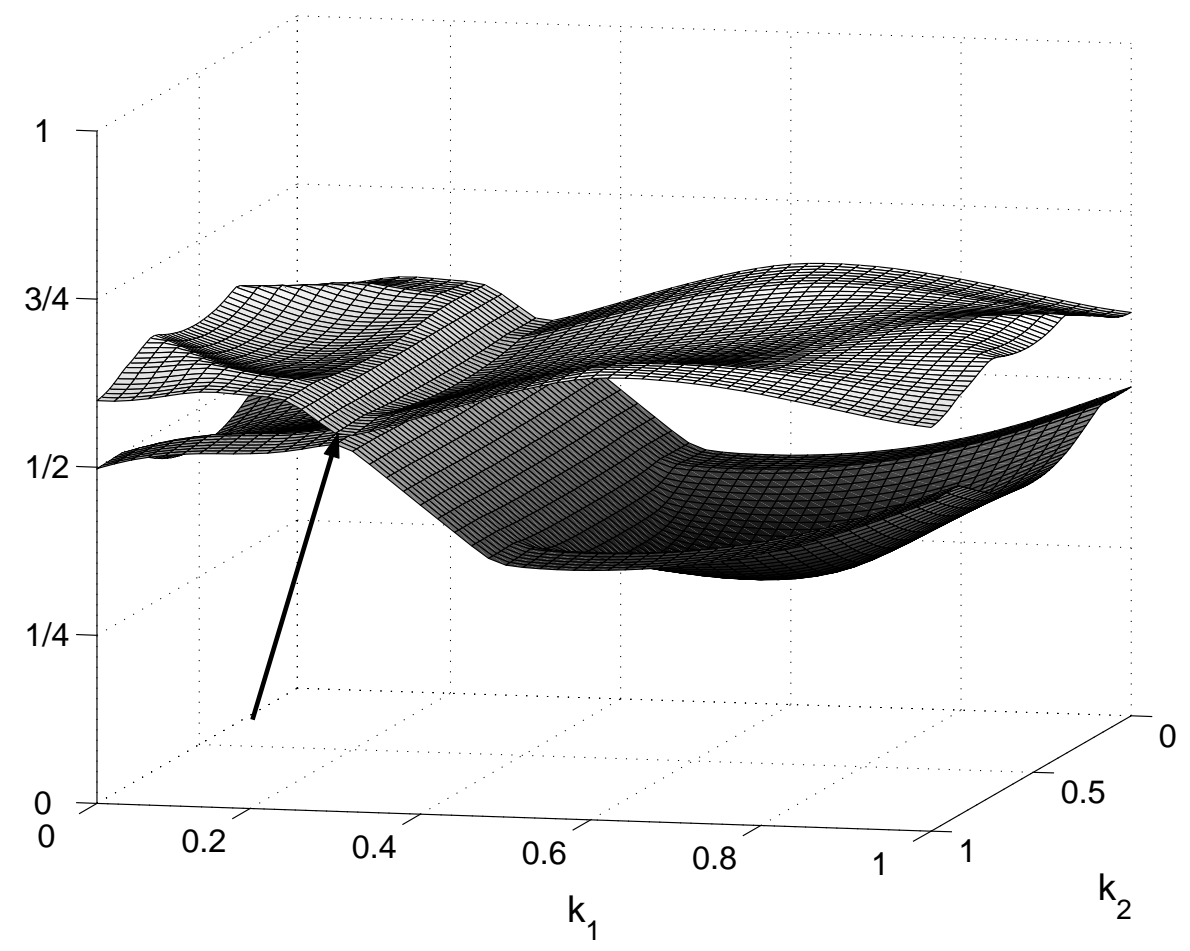

Figure 3.10: Two typical Floquet surfaces $\left.\epsilon_{(} k_{1}, k_{2}\right)$ for the quantum ratchet Eq. 3.25 with $h=1 / 9$. The arrow points at an avoided crossing which is due to the transmission of the regular character from the upper to the lower surface.

lead to a second Bloch phase in the spatial coordinate. The quasienergies now depend on two parameters $k \equiv \theta_{1}$ and $\theta_{2}$, resulting in Floquet surfaces of quasienergies $\epsilon=\epsilon\left(\theta_{1}, \theta_{2}\right)$ which, as shown in Ch. 3.1.2, generically do not cross (cf. Fig. 3.10). The Chern index of surface $\alpha$ is given by

$$
C_{\alpha}=\frac{i}{2 \pi} \int_{0}^{1} \int_{0}^{1} \mathrm{~d}^{2} \theta \sum_{\beta \neq \alpha} \sum_{\mathrm{j}, \mathrm{k}} \mathrm{e}_{\mathrm{jk}} \frac{\left(\partial \mathrm{U} / \partial \theta_{\mathrm{j}}\right)_{\alpha \beta}\left(\partial \mathrm{U} / \partial \theta_{\mathrm{k}}\right)_{\beta \alpha}}{\left|\mathrm{e}^{\mathrm{i} \epsilon_{\alpha}}-\mathrm{e}^{\mathrm{i} \epsilon_{\beta}}\right|^{2}}+\frac{1}{\mathrm{~N}},
$$

where $\left(\partial U / \partial \theta_{j}\right)_{\alpha \beta}=<\psi_{\alpha}\left|\partial U / \partial \theta_{j}\right| \psi_{\beta}>, \mathrm{e}_{j k}$ denotes the completely antisymmetric tensor, $N=1 / h$ is the size of the reduced Hilbert space, and $j, k \in 0,1$.

In the absence of ballistic transport, and for small perturbations of integrability, the Floquet surfaces can be categorized into regular and chaotic. Now, as stated in Ch. 3.1.2, the variation of the Bloch phases corresponds to varying the boundary conditions. With an appropriate choice of the unit-cell boundaries, the regular states supported by nontransporting islands have exponentially small tails at these boundaries, and will therefore not be affected by such a variation. Since the Chern index depends on the derivatives with respect to the boundary conditions, it vanishes for the Floquet surfaces corresponding to such states. This does not apply to a chaotic state, i.e. the Chern index of the corresponding Floquet surface does not necessarily vanish. It can be shown for the kicked Harper map 

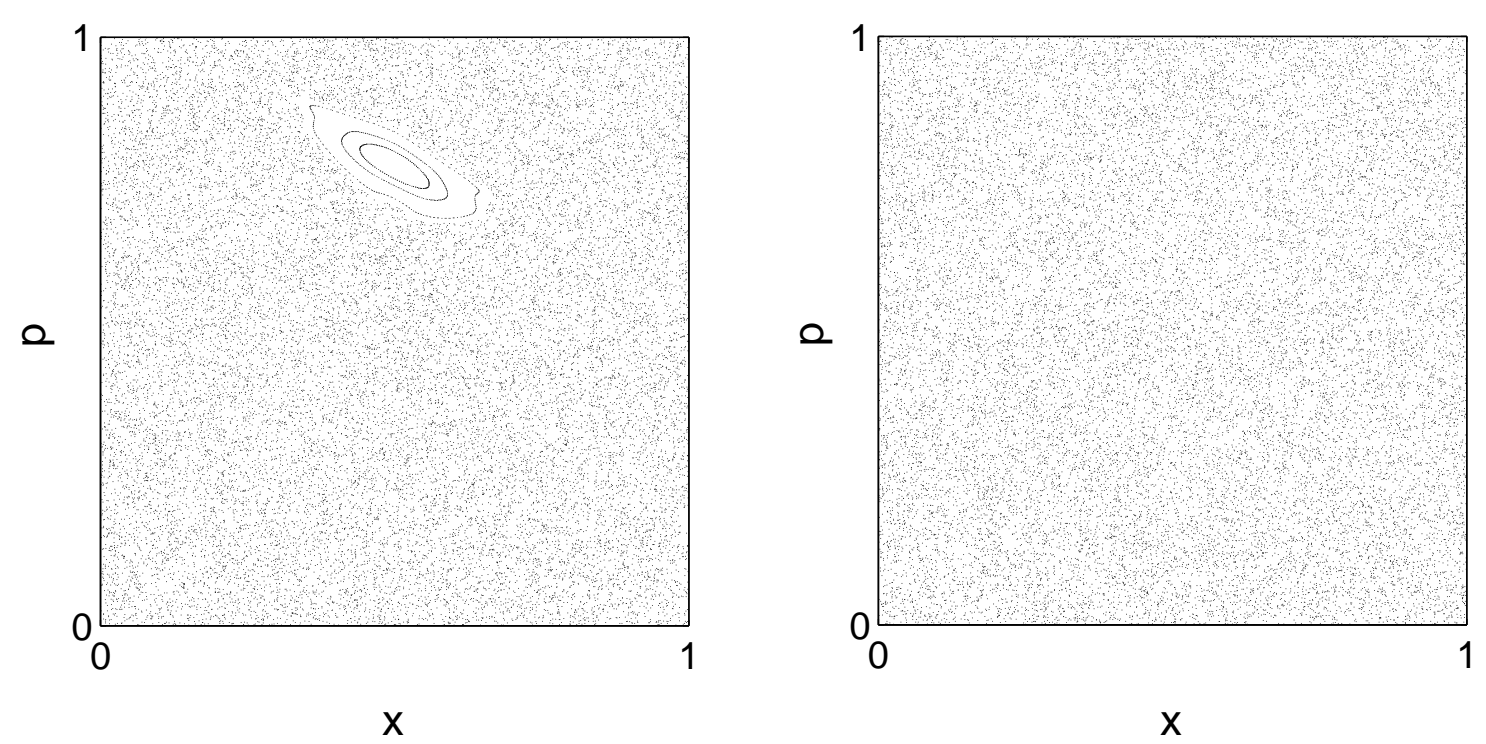

Figure 3.11: Poincaré sections for Eq. 3.25 with parameters leading to a Hamiltonian ratchet (left) and full chaoticity, resp. (right).

that the distribution of Chern numbers around zero is broadened when the perturbation parameter $k=l$ is increased [93]. Hence, the distribution width can be interpreted as a measure for the fraction of states that spread over the chain of unit cells.

The Floquet surfaces of systems which exhibit ballistic transport, however, can not be associated to certain types of eigenstates due to the avoided crossings. Hence, they can not be categorized into regular and chaotic which is a prerequisite of the above statement. One might ask whether it is again possible to reconnect the surfaces in the diabatic approximation. However, the difference of the quasienergies at an avoided crossing decreases exponentially with $h$. Since this difference shows up in the denominator of Eq. (3.24), the avoided crossings can not be neglected in the semiclassical limit. One might still wonder whether transport shows up as a significant broadening of the distribution of Chern indices. This will be investigated in the following.

Since in Eq. (3.24) derivatives of the Floquet operator come into play, one has to employ a model with a smooth potential and kinetic energy. For the variant of the Chirikov map with

$$
\begin{aligned}
V(x) & \left.=\cos (2 \pi \mathrm{x})+0.15 \cos \left(4 \pi \mathrm{x}-\mathrm{a}_{1}\right)+1 / 12 \cos \left(6 \pi \mathrm{x}-\mathrm{a}_{2}\right)\right) \\
T(p) & =\frac{(p+1 / 2)^{2}}{2},
\end{aligned}
$$

$\left(a_{1}, a_{2}\right)=(3 \pi, \pi)$, the Poincaré section shows an asymmetric transporting island with velocity $w_{i}=1$ (see Fig. 3.11).

The distribution of Chern indices is shown in Fig. 3.12 along with the values for a similar 

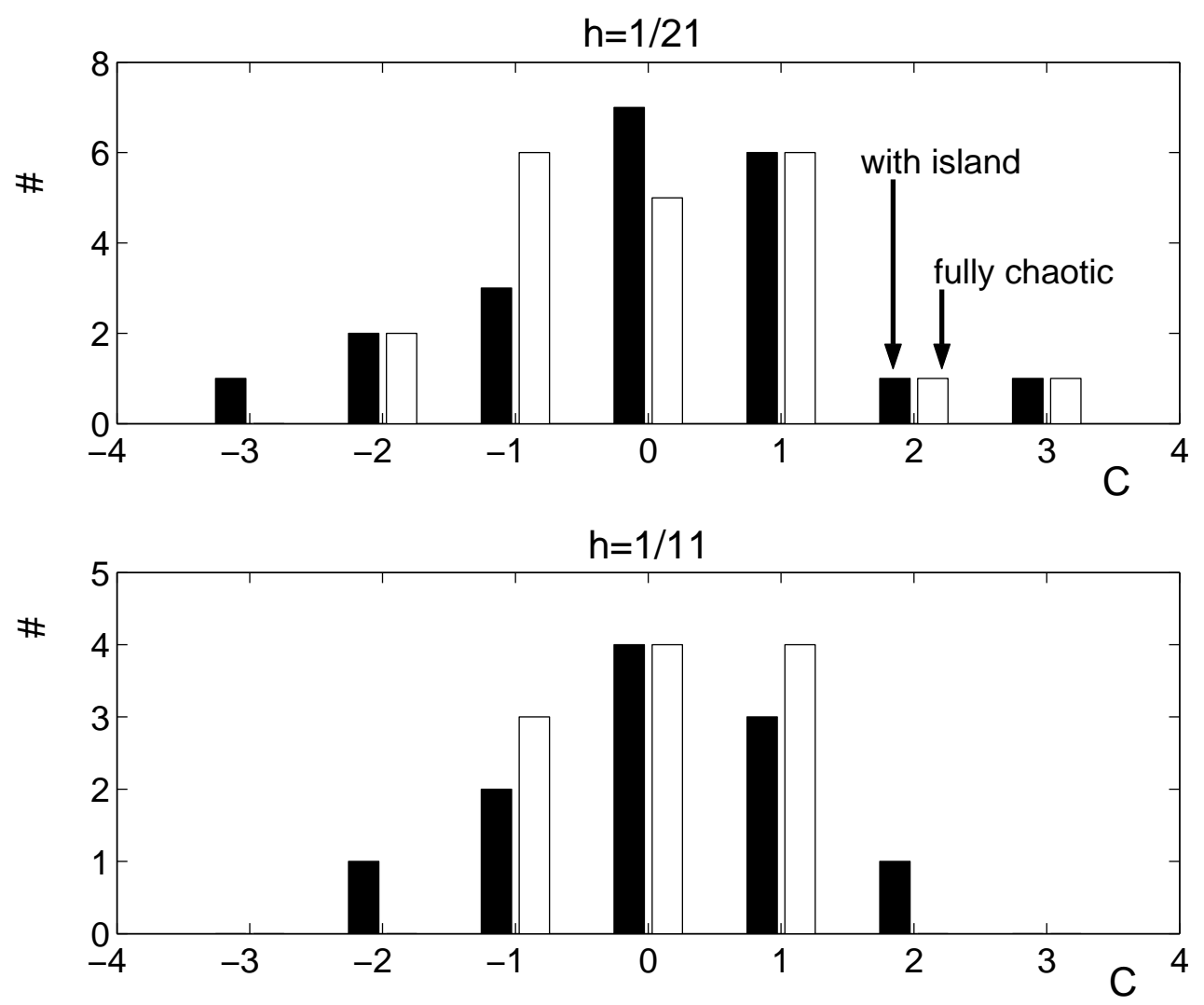

Figure 3.12: Distributions of Chern numbers at different values of $h$ and for both the ratchet Eq. (3.25) and its fully chaotic counterpart. At $h=1 / 11$ the variances $\sigma_{\text {rat }}^{2} \approx 1.29, \sigma_{\text {ch }}^{2} \approx 0.69$ exhibit a larger difference than at $h=1 / 21\left(\sigma_{\text {rat }}^{2} \approx 1.95, \sigma_{\text {ch }}^{2} \approx 1.65\right)$, although the island can only be resolved in the latter case.

but fully chaotic system given by $\left(a_{1}, a_{2}\right)=(2 \pi, 2 \pi)$. The classical island is clearly resolved in the case $h=1 / 21$ but not for $h=1 / 11$. According to the statement by Leboeuf et al. this distribution should be significantly broadened as soon as the systems supports transport. One can indeed observe such a broadening, which, however, also applies to the fully chaotic system. This suggests that the distribution width can not give any indication about the onset of ballistic transport.

Concluding, it is important to note that the concept of conducting chaotic and isolating regular bands is not applicable to Hamiltonian ratchets since there, both types of phasespace regions support eigenstates whose velocity expectation values do not vanish. 


\subsection{Outlook}

It has been shown how a perfect quantum ratchet with compact phase space can make a wave packet centered in the chaotic region with zero initial velocity move at a predetermined velocity which approches the classical value as $h \rightarrow 0$. It might be rewarding to extend the theory to quantum ratchets with a non-compact phase space, or to those with a continuous time evolution.

Choosing a much smaller effective Planck's constant leads to the regime where the classically hierarchical phase-space regions should show up in the quantum dynamics. According to the generalization of the Hellman-Feynman theorem, one would expect the slopes of those bands which correspond to hierarchical states to be close to the slopes of the corresponding regular bands. This calculation could be carried out with a numerical effort smaller than that needed for calculaing the states themselves.

For systems with several islands transporting at different velocities, the corresponding bands should show different slopes with narrow avoided crossings. It might be interesting to extend the study of quantum tunneling to such systems. 


\section{Chapter 4}

\section{Disordered Quantum Hamiltonian Ratchets}

This chapter is concerned with quantized kicked maps where the effective Planck's constant is an irrational number, $h \notin \mathbb{Q}$. Then, as mentioned in Ch. 3.1.2, the spatial symmetry is not preserved by the quantization, and neither Bloch's theorem nor the subsequent Floquet-band analysis apply.

Rather, as will be clarified in the first section, kicked maps with an irrational $h$ are closely related to disordered systems as exemplified by the Anderson model [95]. In particular, the property of localized eigenstates of disordered systems is inherited by the maps. The second section represents an outline of the hypothesis that the eigenstates are supported by the classically invariant phase-space components in the semiclassical limit. For Hamiltonian ratchets, however, one observes the new class of amphibious eigenstates, which ignore the classical phase-space structure completely, as shown and explained in the third section. The understanding of these states also contributes to the study of ballistically transporting systems with time-reversal symmetry like the standard map with accelerator modes (fourth section). Finally, the new insight is applied to the description of the general wave-packet dynamics in Hamiltonian ratchets.

\subsection{Dynamical Localization}

The simplest model suitable for the description of localization is the Anderson model which consists of a chain of sites and a particle which can hop from one site to its $r$ th neighbor with probability $W_{r}^{2}$. At site $m$ acts a random potential $V_{m}$ while $u_{m}$ represents the amplitude of the particle being located at site $m$. One obtains the Schrödinger equation 
as

$$
V_{m} u_{m}+\sum_{r} W_{r} u_{m+r}=E u_{m}
$$

[81] Ch. 7.2. For analytical purposes, this is further simplified by the restrictions that the particle can only hop to the nearest neighbor site and that the hopping is symmetric, i.e. hopping to the left has the same amplitude as hopping to the right.
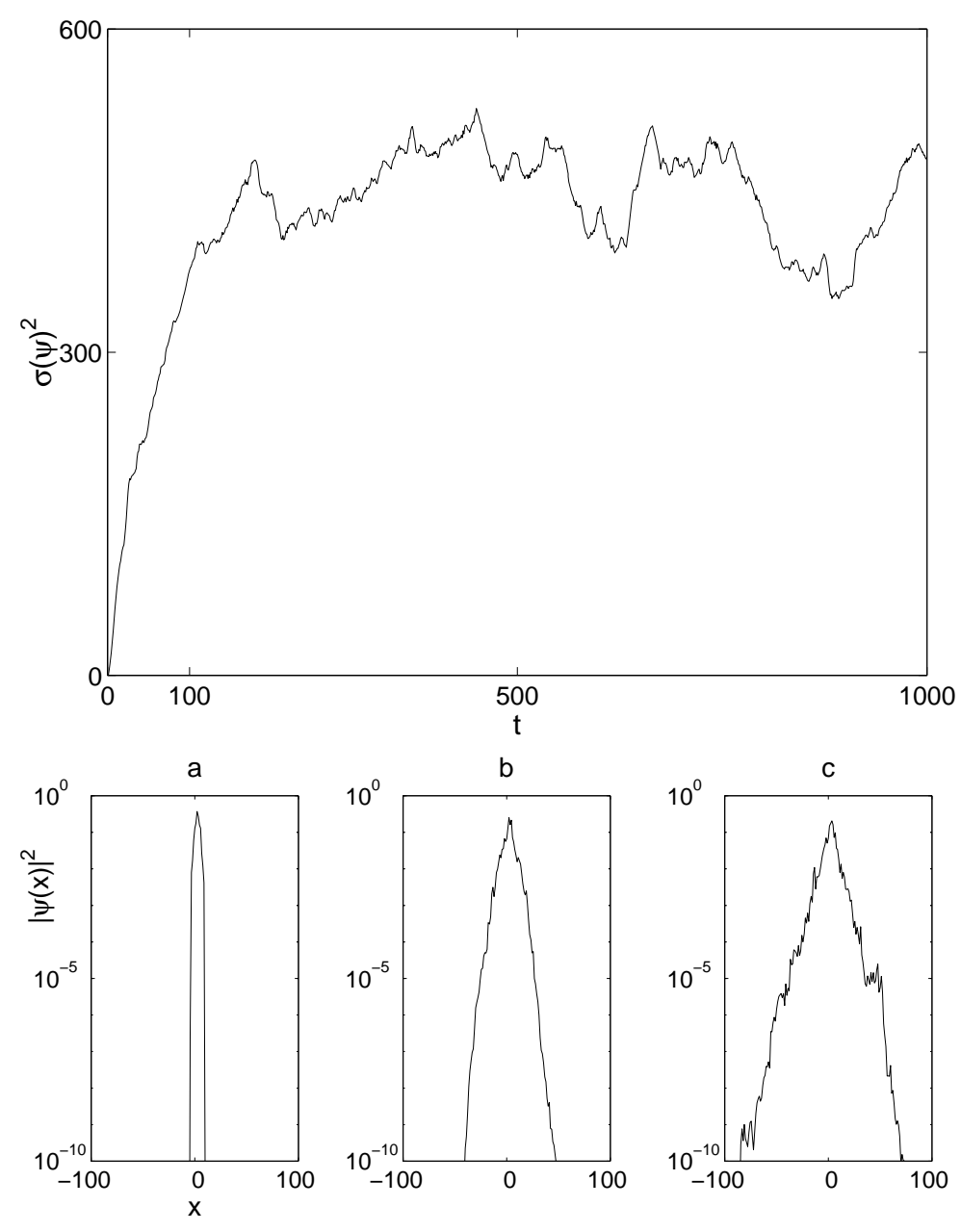

Figure 4.1: (top) Variance of a coherent wavepacket spreading over the phase space of the standard map at $k \approx 1.65$.

(bottom) Momentum representation (or, upon exchanging $x$ and $p$, position representation, respectively) of the wavepacket at times $t=10$ (a), $t=100$ (b), and $t=1000$ (c). The localized wavepacket (c) exhibits exponential decay, justifying the notion of exponential localization.

The Schrödinger equation (4.1) can be written for a two-site vector as

$$
\left(\begin{array}{c}
u_{m+1} \\
u_{m}
\end{array}\right)=\left(\begin{array}{cc}
\left(E-V_{m}\right) / W & -1 \\
1 & 0
\end{array}\right) \equiv M_{m}\left(\begin{array}{c}
u_{m} \\
u_{m-1}
\end{array}\right),
$$


with the transfer matrix $M_{m}$, $\operatorname{det}\left(\mathrm{M}_{\mathrm{m}}\right)=1$. Iterating the transfer yields

$$
\left(\begin{array}{c}
u_{m+1} \\
u_{m}
\end{array}\right)=M_{m} M_{m-1} \ldots M_{2} M_{1}\left(\begin{array}{c}
u_{1} \\
u_{0}
\end{array}\right)
$$

Now, the Fürstenberg theorem [96] states that for long chains, the logarithm of the trace of the iteration matrix is proportional to the length of the iteration,

$$
\lim _{Q \rightarrow \infty} \frac{1}{Q} \log \operatorname{Tr}\left\{\mathrm{M}_{\mathrm{Q}} \mathrm{M}_{\mathrm{Q}-1} \ldots \mathrm{M}_{2} \mathrm{M}_{1}\right\}=\gamma>0
$$

for random matrices $M_{i}$ with $\operatorname{det}\left(\mathrm{M}_{\mathrm{i}}\right)=1$, which is fulfilled by the transfer matrices. A two-by-two matrix with determinant equal to one and an eigenvalues $a$ has a second eigenvalue $1 / a$. One therefore obtains in the long-chain limit the eigenvalues $\exp ( \pm \mathrm{m} \gamma)$ for the product of transfer matrices. It follows that almost all initial vectors $\left(\begin{array}{l}u_{1} \\ u_{0}\end{array}\right)$ will give rise to wave functions which grow exponentially both to the left and to the right. Normalization, however, picks out those states which show exponential decay to both sides. Since these are discrete in the space of all $\left(\begin{array}{l}u_{1} \\ u_{0}\end{array}\right)$, the corresponding spectrum of eigenenergies is also discrete.

Fishman, Grempel, and Prange [50] showed that the standard map with irrational $h$ is related to this model [97]. The notion of dynamical localization - in contrast to Anderson localization - reminds one of the differences between the two model systems: On the one hand, the Floquet operator of the mapping in position representation is not really a random matrix, but only slightly "randomized" by the irrationality of $h$. On the other hand, rather than the hopping being restricted to the next lattice site, its amplitude falls off exponentially for large $r$ in the standard map. Still, the numerical observations have consistently indicated that the eigenstates of the standard map quantized with $h \notin \mathbb{Q}$ are exponentially localized with some localization length $\xi$. That is to say, the spatial weight distribution of these states is given by $|\psi(x)|^{2} \sim \exp (-\mathrm{x} / \xi)$. (With respect to numerics, the irrationality of $h$ is commonly achieved by approximating the number $h=\frac{1}{n+\sigma}$, where $\sigma=\frac{\sqrt{5}-1}{2}$ is the golden mean.)

This leads to a sharp contrast between the quantum dynamics and that of the system's classical version: whereas a bundle of classical trajectories initialized in the chaotic sea exhibits spatial (in the presence of accelerator modes, anomalous) diffusion for all times, any wave packet that can be constructed from a superposition of localized eigenstates will only follow the classical diffusion for times smaller than some break time of the order of the Heisenberg time $t_{H} \sim 1 / h$ and localize thereafter (Fig. 4.1). This behavior is confirmed by a lot of numerical work (see references in [54]). 


\subsection{The Semiclassical Eigenfunction Hypothesis}

The distinction of the eigenstates and corresponding eigenvalues of the time-evolution operator into regular and chaotic (see Ch. 3.1.4) can be stated more explicitly in the semiclassical limit $h \rightarrow 0$ in terms of the Wigner function. This semiclassical eigenfunction hypothesis is based mainly on work by Percival [40], Berry [41,42], Voros [98], and Shnirelman [99]. Stated originally in terms of time-independent $N$-dimensional systems with conjugate variables $\mathbf{x}$ and $\mathbf{p}$, the hypothesis also applies to the two-dimensional stroboscopic section of kicked maps as investigated in this thesis.

One starts out from the locally averaged Wigner function

$$
\bar{\Psi}(\mathbf{x}, \mathbf{p})=\frac{1}{\Delta} \int_{\mathbf{x}^{\prime}-\Delta / 2}^{\mathbf{x}^{\prime}+\Delta / 2} \mathrm{~d} \mathbf{x}^{\prime} \int_{\mathbf{p}^{\prime}-\Delta / 2}^{\mathbf{p}^{\prime}+\Delta / 2} \mathrm{~d} \mathbf{p}^{\prime} \Psi\left(\mathbf{x}^{\prime}, \mathbf{p}^{\prime}\right),
$$

where $\lim _{\hbar \rightarrow 0} \Delta=0$, but $\lim _{\hbar \rightarrow 0}(\hbar / \Delta)=0$ [42], such that the oscillations, whose length scales with $h$, are smoothed out. In a classically integrable system, the canonical transformation to action-angle variables yields the invariant actions $I$, which can be expressed in terms of the original coordinates $\mathbf{x}$ and $\mathbf{p}, I=I(\mathbf{x}, \mathbf{p})$. Then, the averaged Wigner function of state $\psi$ is given in the semiclassical limit by [41]

$$
\bar{\Psi}(\mathbf{x}, \mathbf{p})=\frac{\delta^{N}\left(I(\mathbf{x}, \mathbf{p})-I_{\psi}\right)}{(2 \pi)}
$$

where $I_{\psi}$ denotes the action values of $\psi$, and $\delta^{N}$ is the $N$-dimensional Dirac delta function. That is to say, the state is confined to the classical torus of corresponding action.

For an ergodic system and with the classical Hamiltonian $H(\mathbf{x}, \mathbf{p})$, Voros [98] hypothesized that for almost all states $\psi$ and $h \rightarrow 0$,

$$
\bar{\Psi}(\mathbf{x}, \mathbf{p})=\frac{\delta^{1}\left(E_{\psi}-H(\mathbf{x}, \mathbf{p})\right)}{\int \mathrm{d} \mathbf{x} \int \mathrm{d} \mathbf{p} \delta^{1}\left(E_{\psi}-H(\mathbf{x}, \mathbf{p})\right)},
$$

i.e. the state is spread evenly over the whole energy shell. This statement, proven later by Shnirelman [99], is referred to as the quantum ergodicity or Shnirelman theorem. The analogy of the Wigner functions in these two extremal cases suggests the expression,

$$
\bar{\Psi}(\mathbf{x}, \mathbf{p})=\frac{\delta\left(F_{\psi}-F(\mathbf{x}, \mathbf{p})\right) \chi_{\omega}(\mathbf{x}, \mathbf{p})}{\int \mathrm{d} \mathbf{x} \int \mathrm{d} \mathbf{p} \delta\left(F_{\psi}-F(\mathbf{x}, \mathbf{p})\right) \chi_{\omega}(\mathbf{x}, \mathbf{p})},
$$

for a state $\psi$ of a classically mixed system with ergodic chaotic components. Here, $F(\mathbf{x}, \mathbf{p})$ is a generalized constant of motion, $F_{\psi}$ denotes the corresponding quantum value and $\chi_{\omega}$ represents the characteristic function of the phase-space component $\omega$ defined by a certain value of $F[100]$. With small $h$ but $h \neq 0$, the Wigner function broadens, the width of 
the peak scaling with $\hbar^{-2 N / 3}$ [41], leading to a small overlap of the Wigner functions for eigenstates localized on different invariant manifolds.

The semiclassical eigenfunction hypothesis is supported by convincing numerical and experimental data [44-46]. It forms the basis of the present understanding of spectral and transport properties of quantum systems with coexisting regular and chaotic classical dynamics $[42,47,60]$. Therefore, any limitation of its applicability is of great interest for the field of quantum chaos.

\subsection{Broken Time-Reversal Symmetry}

The maximum violation of the semiclassical eigenfunction hypothesis would be achieved when all eigenstates of a system completely ignore the classical phase space structure in the semiclassical limit. These conditions are met by the eigenstates of quantum Hamiltonian ratchets with broken time-reversal symmetry as studied in this section. In the first subsection, the eigenstates are introduced and their localization properties are described. From the continuity equation for the quantum current, an argument for the states' local structure is derived in the second subsection, justifying the statement of maximum violation of the semiclassical eigenfunction hypothesis. Finally, it is shown why the perturbation theory leading to this hypothesis fails in the presence of transporting islands.

\subsubsection{Amphibious Eigenstates}

Fig. 4.2 A shows an example of an amphibious eigenstate living both on the regular island and the chaotic sea. The comparison with the eigenstates of a similar system with nontransporting islands at the same $h$ shows that the quantum resolution is high enough to permit the distinction into regular and chaotic states (cf. Fig. 4.2) B, C. Amphibious eigenstates should not be confused with the well-known occurrence of partly regular and partly chaotic eigenstates due to avoided crossings. These are simple superpositions of regular and chaotic eigenstates and they occur with zero probability in the semiclassical limit. It should be noted, that although amphibious eigenstates ignore the classical phasespace structure they conspire in such a way that the dynamics of wave packets follows the semiclassical expectation. For example, a wave packet started in the regular island follows the classical motion of this island to very high precision (see Fig. 4.3). 


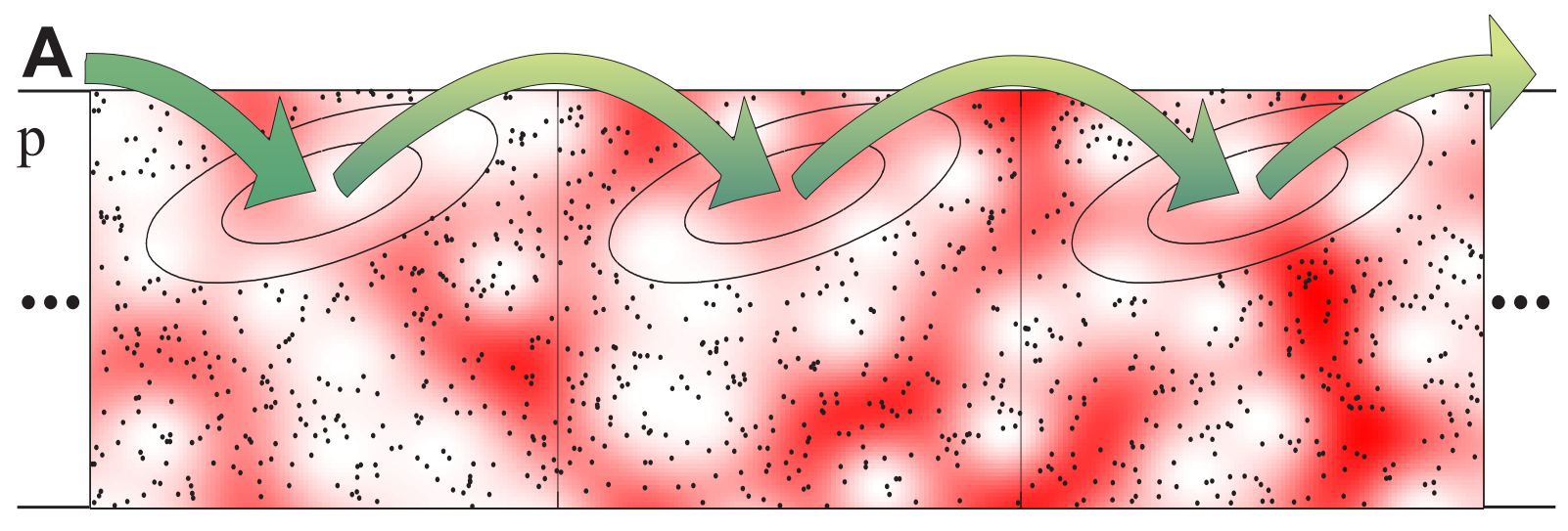

$\mathrm{X}$
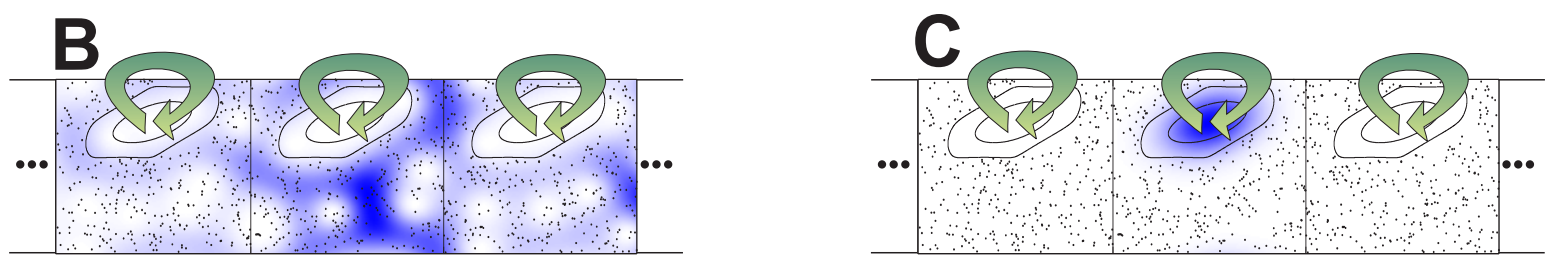

Figure 4.2: Husimi representations of eigenstates of a transporting (A) and an analogous nontransporting system (B), (C) as indicated by the arrows. The classical phase space portraits are shown in black. (A) All eigenstates, like the one shown, spread over the classically regular islands as well as the chaotic sea. These "amphibious" eigenstates ignore the classical structures even though $h$ is smaller than the island. For comparison, the well-known eigenstates which can be categorized into chaotic (B) and regular (C) are shown. These are in agreement with the semiclassical eigenfunction hypothesis.

\section{Model Systems}

For both the transporting $(\mathrm{T})$ and the non-transporting (NT) system, one starts from piecewise linear functions

$$
\begin{aligned}
t^{\prime}(p) & =s / 4 \pm(A-s p): 0< \pm p<1 / 2 \\
v^{\prime}(x) & =-(m+r(x-m)): m-1 / 2<x<m+1 / 2,
\end{aligned}
$$

where $m$ is the unit cell index. Smoothing is carried out by folding the discontinuous functions with a Gaussian, $G(z)=\exp \left(-z^{2} / 2 \varepsilon^{2}\right) / \sqrt{2 \pi \varepsilon^{2}}$, finally yielding $T^{\prime}(p)=$ $\int d z t^{\prime}(p+z) G(z)$ and $V^{\prime}(x)=\int d z v^{\prime}(x+z) G(z)$. With the values $A=1, s=2$, $r=0.65$, and $\varepsilon=0.015$, one obtains the classical phase space shown in Fig. $4.2 \mathrm{~A}$ with a transporting island (T), whereas $A=0, s=2, r=0.65$, and $\varepsilon=0.11$ yield a system with non-transporting island (NT) of roughly the same size - approximately 50 times larger than the accelerator modes of the standard map at $K \approx 6.33$. Choosing the effective Planck's constant as $h=1 /(9+\sigma)$ leads to one regular state per unit cell in the case where the semiclassical eigenfunction hypothesis applies. 

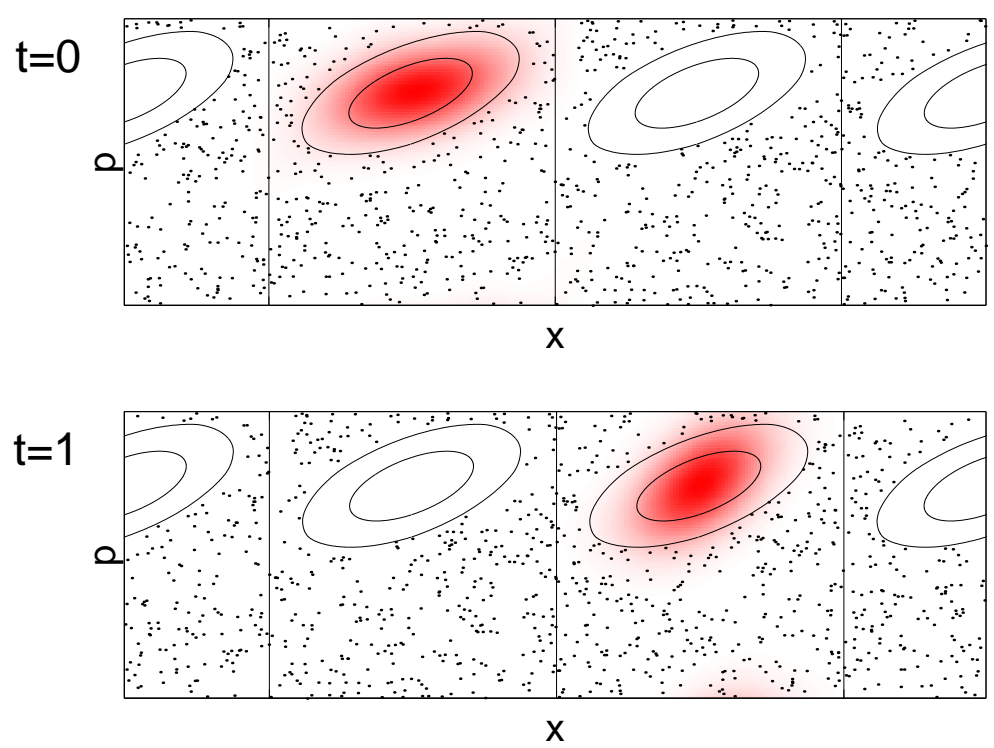

Figure 4.3: Husimi representations (red) of a wave packet initialized on the transporting island (top) and after one iteration (bottom) together with the Poincaré sections of the classical system. Although this wave packet can be expressed as a superposition of amphibious eigenstates only, it follows the semiclassical expectation for short times.

\section{Increased Localization Length}

While the chaotic states of the non-transporting system exhibit a relatively small localization length $\xi_{\mathrm{NT}} \approx 0.22$ (and the regular ones are localized on a single island), the amphibious states show a greatly increazed value, $\xi_{\mathrm{T}} \approx 1.01 \cdot 10^{3}$ (Fig. 4.4), as anticipated from previous studies of wave-packet dynamics $[56,57,86]$. Starting a wave packet at the center of the transporting island with classical winding number $w_{i}=1$, one can determine the tunneling rate $\gamma$ from the decay of the weight in the transported island $P(t)=\exp (-\gamma t)$ [86]. During the time scale $1 / \gamma$ of this decay the wave packet is transported over $w_{i} / \gamma$ spatial unit cells. Hence, one estimates a dynamical localization length as $\xi=w_{i} / \gamma$. The initial wavepacket can be expressed in the basis of eigenstates. According to the observation, all the eigenstates have essentially the same exponentially decreasing shape. Then, the scale of the dynamical localization length is recovered in the localization length of the eigenstates, as confirmed in Fig. 4.4.

When numerically modeling an infinitely extended system, the system size must be chosen significantly larger than the localization length in order to avoid a strong influence of the boundary conditions. On the other hand, it can be shown that $\gamma$ decreases exponentially with $h$ [86], as visualized in Fig. 4.3.1. This explains the enormously increasing numerical effort when going to smaller values of $h$ or, more precisely, to larger ratios of island size over quantum parameter $A_{\text {island }} / h$. 


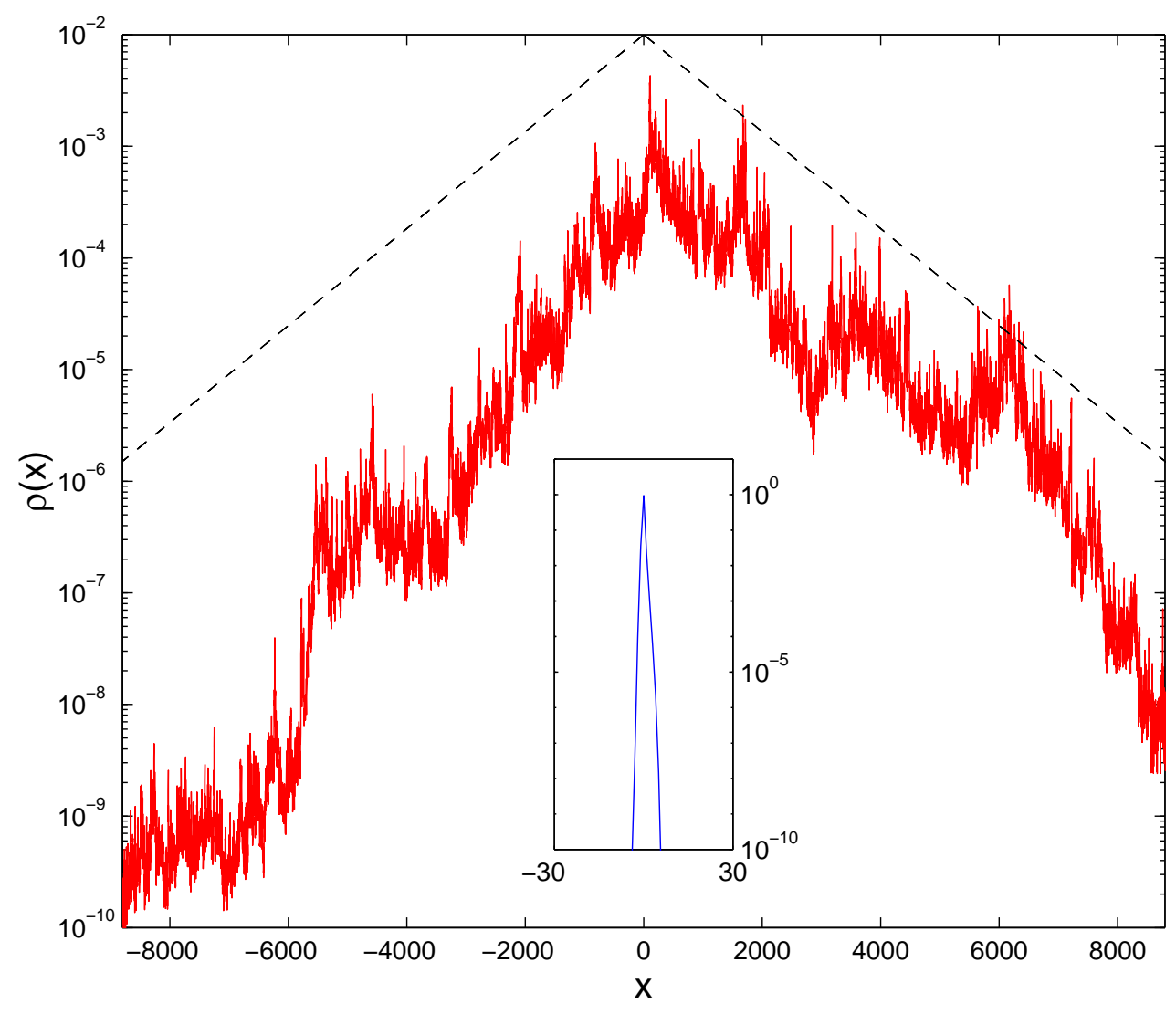

Figure 4.4: Density distribution of the position representation of the amphibious eigenstate shown in Fig. $4.2 \mathrm{~A}$ averaged over unit cells, $\rho(x)=\int_{x-1 / 2}^{x+1 / 2} d x^{\prime}\left|\psi\left(x^{\prime}\right)\right|^{2}$. The localization length is several orders of magnitude bigger than in the non-transporting case of Fig. $4.2 \mathrm{~B}$ (inset). It is quite well estimated by $\exp (-|x| / \lambda)$ (dashed line) with $\lambda=v / \gamma$ determined from the tunneling rate $\gamma$ out of the transporting regular island.

\subsubsection{Local Probability Current}

The observation that the amphibious eigenstates ignore the classical phase space within each unit cell can be explained intuitively: Due to localization, the position representation of an eigenstate has exponentially small weight in its tails. On the other hand, its time evolution over one period may not lead to a probability current in these tails. Hence, the current must vanish also with respect to each unit cell. With broken time-reversal symmetry, this can only be fulfilled if the state is amphibious in each unit cell. This is stated analytically in the following.

The one-dimensional continuity equation of quantum mechanics

$$
\frac{\partial}{\partial t}|\psi(x, t)|^{2}+\frac{\partial}{\partial x} J_{\psi}(x, t)=0
$$




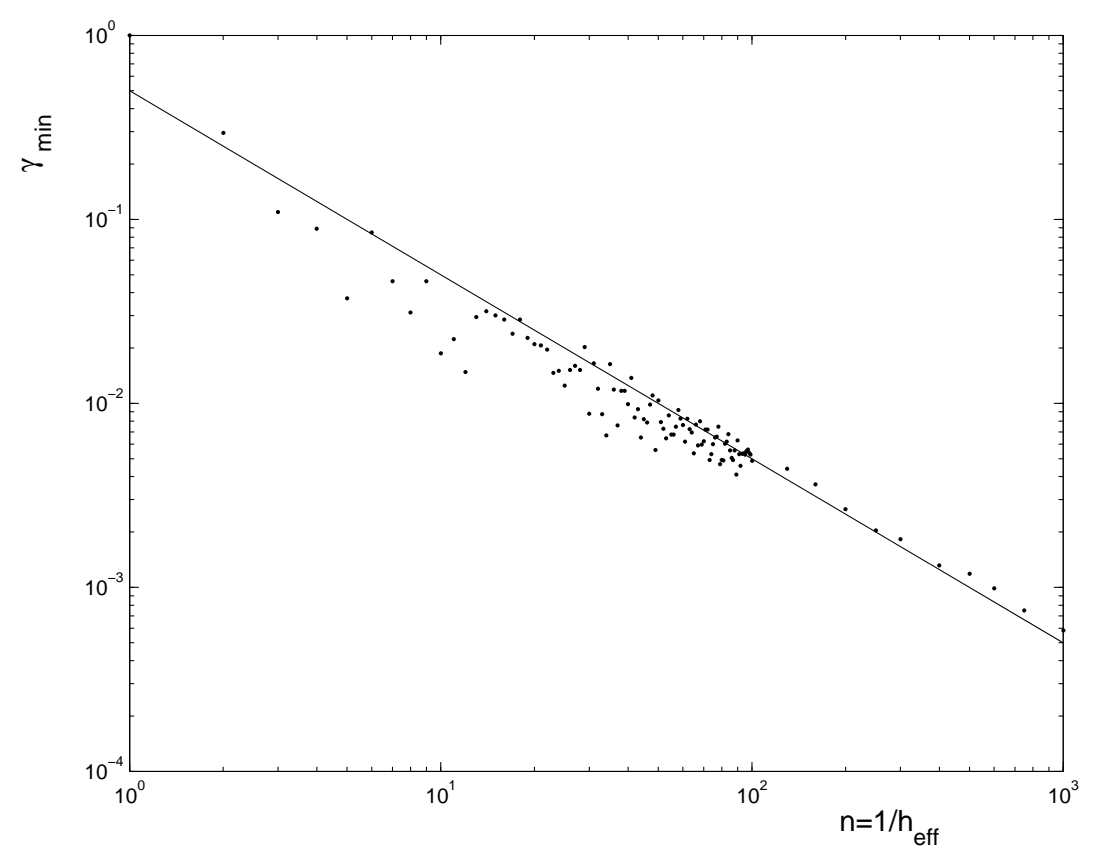

Figure 4.5: Overlap of the eigenstate at the center of the island with a coherent wave packet centered on the hyperbolic fixed point in the chaotic sea as a function of Planck's constant, indicating the exponential decay of $\gamma$ with $1 / h$. The model system is given classically by Eq. 2.31 .

where $J_{\psi}$ is the probability current of $\psi$. Integrating this equation over one temporal period leads to

$$
|\psi(x, t+1)|^{2}-|\psi(x, t)|^{2}=-\frac{\partial}{\partial x} \int_{t}^{t+1} \mathrm{~d} t^{\prime} J_{\psi}\left(x, t^{\prime}\right),
$$

with a left-hand side that vanishes for eigenstates $|\psi\rangle$ of the Floquet operator. Thus the temporally averaged current is spatially constant. Moreover, this constant is zero since for a localized eigenstate, the current vanishes in the tails, $\lim _{x \rightarrow \infty} \int_{t}^{t+1} \mathrm{~d} t^{\prime} J_{\psi}\left(x, t^{\prime}\right)=0$. Hence,

$$
\int_{t}^{t+1} \mathrm{~d} t^{\prime} J_{\psi}\left(x, t^{\prime}\right) \equiv 0 \quad \forall x
$$

In the semiclassical regime this probability current is expected to be close to the classical current, as visualized by the classical and quantum mechanical time-evolution in Fig. 4.3. This excludes regular eigenstates that mainly live on the classically invariant islands since these sets show a considerable non-zero transport. Similarly, chaotic eigenstates can be excluded, since, according to the classical sum rule, the chaotic sea also shows a non-zero current in the opposite direction. The amphibious eigenstate presented in Fig. $4.2 \mathrm{~A}$, however, fulfills the requirement of a vanishing current Eq. (4.12) because the phase space averaged classical current is zero. Hence one concludes that even in the semiclassical limit all eigenstates spread simultaneously over regular and chaotic regions of phase space in all 
unit cells.

\subsubsection{Perturbation Theory of Mixed Phase Space}

It is illuminating to point out where the arguments leading to the semiclassical eigenfunction hypothesis fail for systems with transporting islands. In the classical limit, the tunneling rate $\gamma$ between the center of a regular island and the chaotic sea vanishes. For small $h$ but $h \neq 0$, this suggests a perturbation theory in $\gamma$ : the Hamiltonian is written as $H=H_{0}+H_{1}$, where $H_{1}$ couples the chaotic and regular eigenstates, $\phi^{(\mathrm{reg})}$ and $\phi^{(\mathrm{ch})}$, of $H_{0}$. An eigenstate $\psi$ of $H$ is given to first order by

$$
\left|\psi_{j}\right\rangle=\left|\phi_{j}\right\rangle+\sum_{k \neq j} \frac{\left\langle\phi_{k}\left|H_{1}\right| \phi_{j}\right\rangle}{E_{j}-E_{k}}\left|\phi_{k}\right\rangle,
$$

provided that $\frac{\left\langle\phi_{k}\left|H_{1}\right| \phi_{j}\right\rangle}{E_{j}-E_{k}} \ll 1, \forall j \neq k$. The denominator is estimated as the mean level spacing $\Delta$ of those unperturbed states that are coupled by $H_{1}$. On the other hand, as follows from Fermi's golden rule, the tunneling rate $\gamma$ is proportional to the square of this quotient times $\Delta$, i.e.

$$
\frac{\gamma}{\Delta}=\left(\frac{\left\langle\phi_{k}\left|H_{1}\right| \phi_{j}\right\rangle}{\Delta}\right)^{2}
$$

Then, the condition for the applicability of perturbation theory can be stated as $\gamma / \Delta \ll 1$.

In the absence of transporting islands, the unperturbed eigenstates $\phi$ are localized, and $H_{1}$ may couple only those groups of states which are spatially close. The number of these states is proportional to the localization length which scales with $1 / h$, such that $\Delta \sim h$. Therefore, with $\gamma \sim \exp (-C / h), \gamma / \Delta \ll 1$ and perturbation theory is applicable. In the presence of transporting islands, however, the unperturbed regular states are extended and all of them will be coupled to all the chaotic states. $\Delta$ now depends crucially on the system size: with a chain of $L$ unit cells, the number of chaotic states is approximately given by $L / h$, leading to $\Delta \sim h / L$. Then, perturbation theory breaks down as soon as $L \sim h / \gamma$. In particular, for infinitely extended systems, i.e. taking the limit $L \rightarrow \infty$ first, the transporting regular and chaotic regions are strongly coupled even in the semiclassical limit. From the breakdown of perturbation theory alone, however, one cannot conclude the locally amphibious nature of eigenstates which is found numerically and which can be deduced from the condition of vanishing current Eq. (4.12).

\subsection{Time-Reversal Symmetric Case}

For ratchets with time-reversal symmetry, a transporting island will have a symmetryrelated counterpart transporting in the opposite direction, like the standard map in the 
regime of accelerator modes $(K \approx 6.33$, and with $x$ and $p$ exchanged to obtain spatial transport). In this case, regular eigenstates that are supported simultaneously by both islands would fulfill the condition of vanishing probability current Eq. (4.12). However, due to the breakdown of perturbation theory, the appearance of such states is not mandatory.

\subsubsection{Eigenstates in the Symmetric Case}

Fig. 4.6 shows a typical eigenstate of a time-reversal symmetric quantum ratchet. The eigenstates are of a mixed type: in some unit unit cells they are concentrated on both dominant regular islands, in some they are confined to the chaotic sea, and in some unit cells they are amphibious. That is to say, the eigenfunctions show all the local structures allowed by the condition of vanishing current.

The model is analogous to the standard map up to the spatial dependence of the potential,

$$
V(\hat{x})=1.22 \cos (2 \pi \hat{x})+0.1 \cos (4 \pi \hat{x})+0.05 \cos (6 \pi \hat{x}) \text {, }
$$

before exchanging $x$ and $p$. It has the same symmetries and transport properties as the standard map with accelerator modes. Its islands, however, are significantly larger which leads to a significantly smaller numerical expense. Due to the close analogy, it is natural to suggest that the standard map's eigenstates exhibit the same mixed type.

\section{Localization Length in the Symmetric Case}

The difference of the probability distribution of the symmetric eigenstate Fig. 4.6 to the asymmetric case Fig. 4.4 consists in the local substructure: relating the probability distribution to the Husimi representation (see Fig. 4.6), one finds sharp peaks for chaotic sections, plateaus with small slopes for regular sections, and intermediate slopes for the amphibious sections. In general, such a substructure allows a great range of scale for the total localization length.

The calculated sample of eigenstates, however, suggests that all the states have the same length scale. Then, each state visits the different phase-space regions in a fraction of unit cells corresponding to their weight. That is to say, on a global scale, the state is equally distributed over the phase-space components, like an amphibious eigenstate is on a local scale. There is no reason to assume that the total localization length of such a state differs from that of an amphibious eigenstate in an asymmetric ratchet with similar transport and tunneling properties.

In order to verify the assumption that time-reversal symmetry is not crucial for the localization length, one can fall back on the analysis of wave-packet dynamics: as shown in 


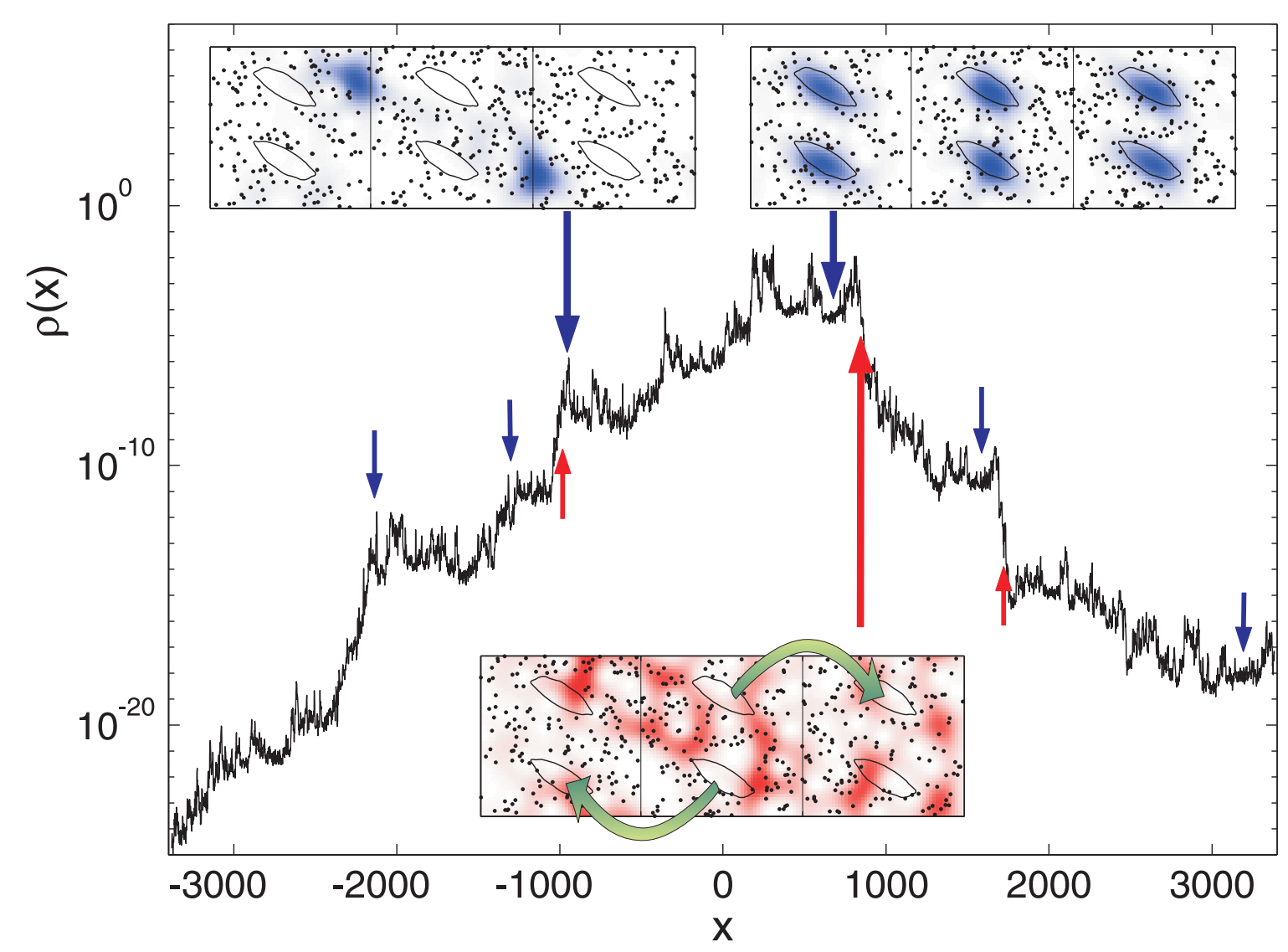

Figure 4.6: Typical eigenstate for the system analogous to the standard map at $k \approx 2 \pi$ with a symmetric phase space and islands transporting in opposite directions. The insets show the Husimi representations (together with the classical Poincaré sections) of the eigenstate at different locations, demonstrating the mixed type: Whereas in some sections, the state is mainly chaotic, it is mainly regular or amphibious in others. Each type appears at many locations (short arrows), leading to different slopes of $\rho(x)$ : In those sections where the state has its weight in the classically chaotic set, the localization length is seen to be much smaller, namely corresponding to the chaotic localization length.

Fig. 4.7, the localization length of the symmetric eigenstate can be approximated, like in the asymmetric case, by the inverse of the tunneling rate of a wavepacket initialized on a regular island. A model which is similar to that with the potential of Eq. (4.15) is given by

$$
V(\hat{x})=\cos (2 \pi \hat{x})+0.15 \cos (4 \pi(\hat{x}-0.75))+1 / 12 \cos (6 \pi(\hat{x}-1 / 6))
$$

Fig. 4.8 shows that the tunneling rates for the two systems are indeed similar for the same $A_{\text {island }} / h$, i.e. the states localize on the same length scale for both systems. 


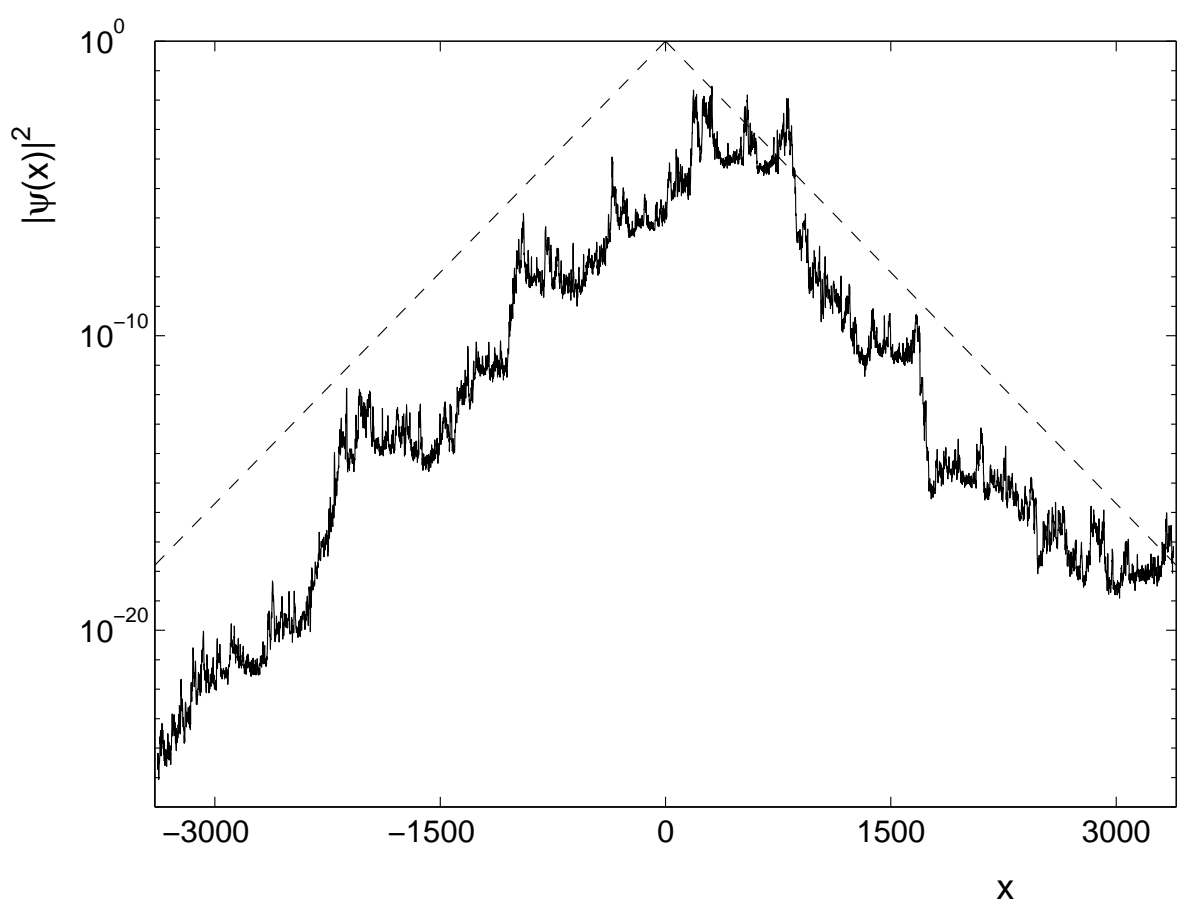

Figure 4.7: $\rho(x)$ from Fig. 4.6 approximated by $\exp (-|x| \gamma)$ (dashed line), where $\gamma$ was determined from the tunneling rate (cf. Ch. 4.3.1).

\subsubsection{Accessing the Critical Parameters with a Graph Model}

The ratio $A_{\text {island }} / h \leq 2$ achieved with the map numerics is far from the semiclassical limit. In this situation, a toy model which allows to investigate all the features of the eigenstates is certainly of interest. Moreover, the ability to construct a mimimal model which still exhibits all the relevant phenomena is a strong support for the theory. Such a model will be presented in the following.

A graph consists of a set of vertices connected by directed bonds. Essentially, the phasespace representation of the real quantum system is associated to the bonds $b_{j}$, whereas the vertices $v_{k}$ determine the dynamical properties. With the goal to simplify the situation as much as possible, the chaotic region of one unit cell is modelled by two directed bonds, and a transporting island by a number of bonds directed parallel to the island's transport direction (see Fig. 4.9 (top)). The chaotic bonds are strongly coupled with each other, but only exponentially weak with the outer regular bonds, in analogy to the exponentially small tunneling rate from the center of a classical island in a kicked map. The coupling of the $l^{\text {th }}$ regular bond to the chaotic bonds is given by $\epsilon^{l}$ with $\epsilon<1$ (Fig. 4.9 (bottom)).

A state $\phi$ of the system is a vector with the length given by the total number of bonds; $\left|\phi\left(b_{j}\right)\right|^{2}$ denotes the state's weight at bond $b_{j}$. Modelling the time evolution, $\phi$ is multiplied 


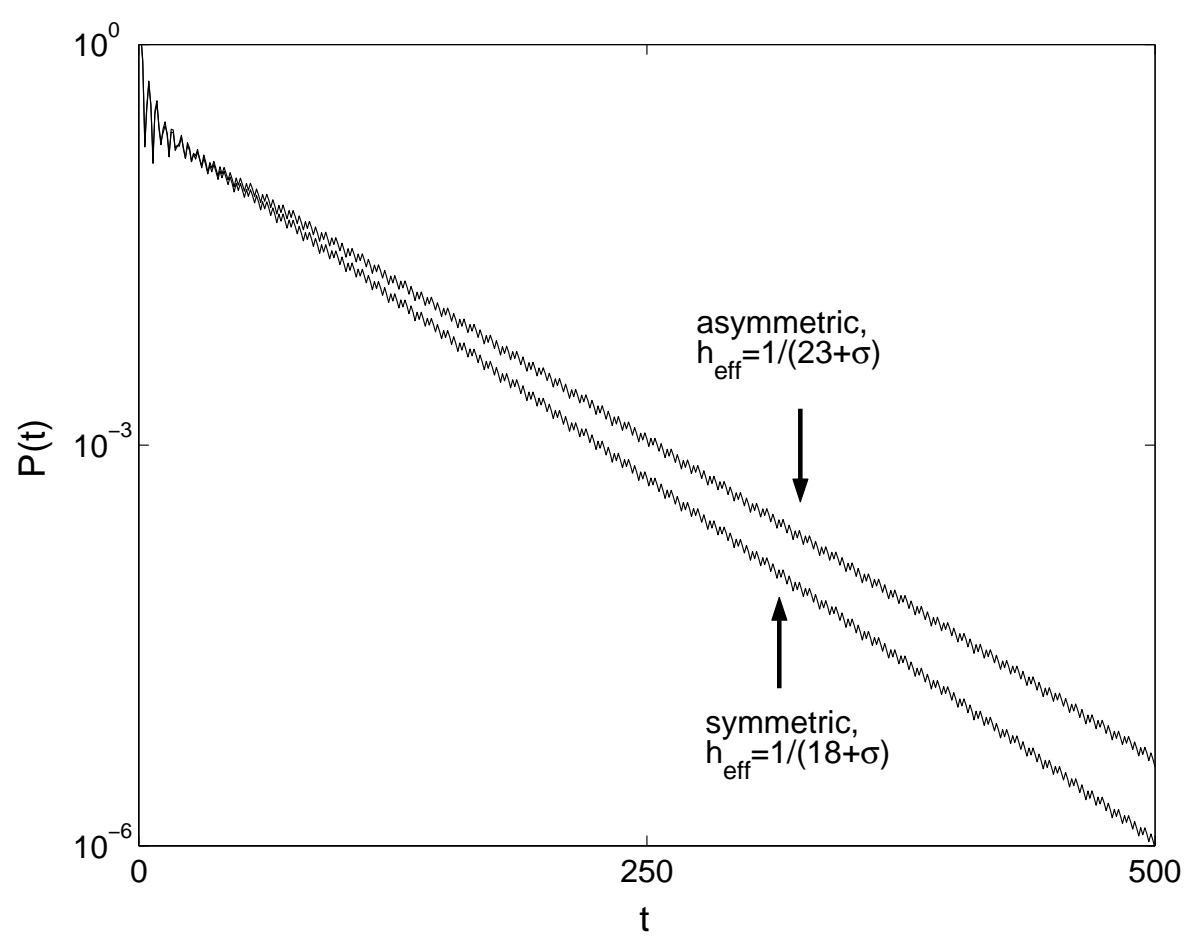

Figure 4.8: Determination of $\gamma$ from $P(t)=\exp (-\gamma t)$ (cf. Ch. 4.5.2) for the two systems shown classically in Fig. 2.4 (Eqs. (4.15) and (4.16)). With the size of one island determined classically from the last torus, $A_{\text {island }}^{\text {symm. }} \approx 0.042, A_{\text {island }}^{\text {asymm. }} \approx 0.039, h$ is chosen such that $A_{\text {island }} / h$ is approximately the same in both cases.

with the unitary matrix which contains the coupling information of the vertices. The construction of this matrix consists of three steps: First, one sets up a hermitian matrix $M$, corresponding to one node. Combining all the bonds which are incoming from (or outgoing to, respectively) the same direction yields four sets of bonds at the node. Then, $M$ can be decomposed into four equally large quadratic sub-matrices,

$$
M=\begin{array}{c|cc} 
& \text { from left } & \text { from right } \\
\hline \text { to left } & A & B \\
\text { to right } & B & A
\end{array},
$$

where the sub-matrix $R$ contains the elements for reflection at the node (from the left to the left, or from the right to the right), whereas $T$ amounts to a transition. The coupling rates, are given by the squared matrix elements. Therefore, choosing $A$ and $B$ as

$$
A=\frac{1}{\sqrt{2}}\left(\begin{array}{ccc}
1 & \epsilon & \epsilon^{2} \\
\epsilon & 0 & 0 \\
\epsilon^{2} & 0 & 0
\end{array}\right), B=\frac{1}{\sqrt{2}}\left(\begin{array}{ccc}
1 & \epsilon & \epsilon^{2} \\
\epsilon & \sqrt{2} & 0 \\
\epsilon^{2} & 0 & \sqrt{2}
\end{array}\right),
$$

fulfills the conditions on the coupling rates shown in (Fig. 4.9 (bottom)). 


$$
\mathrm{m}-1 \quad \mathrm{~m} \quad \mathrm{~m}+1
$$
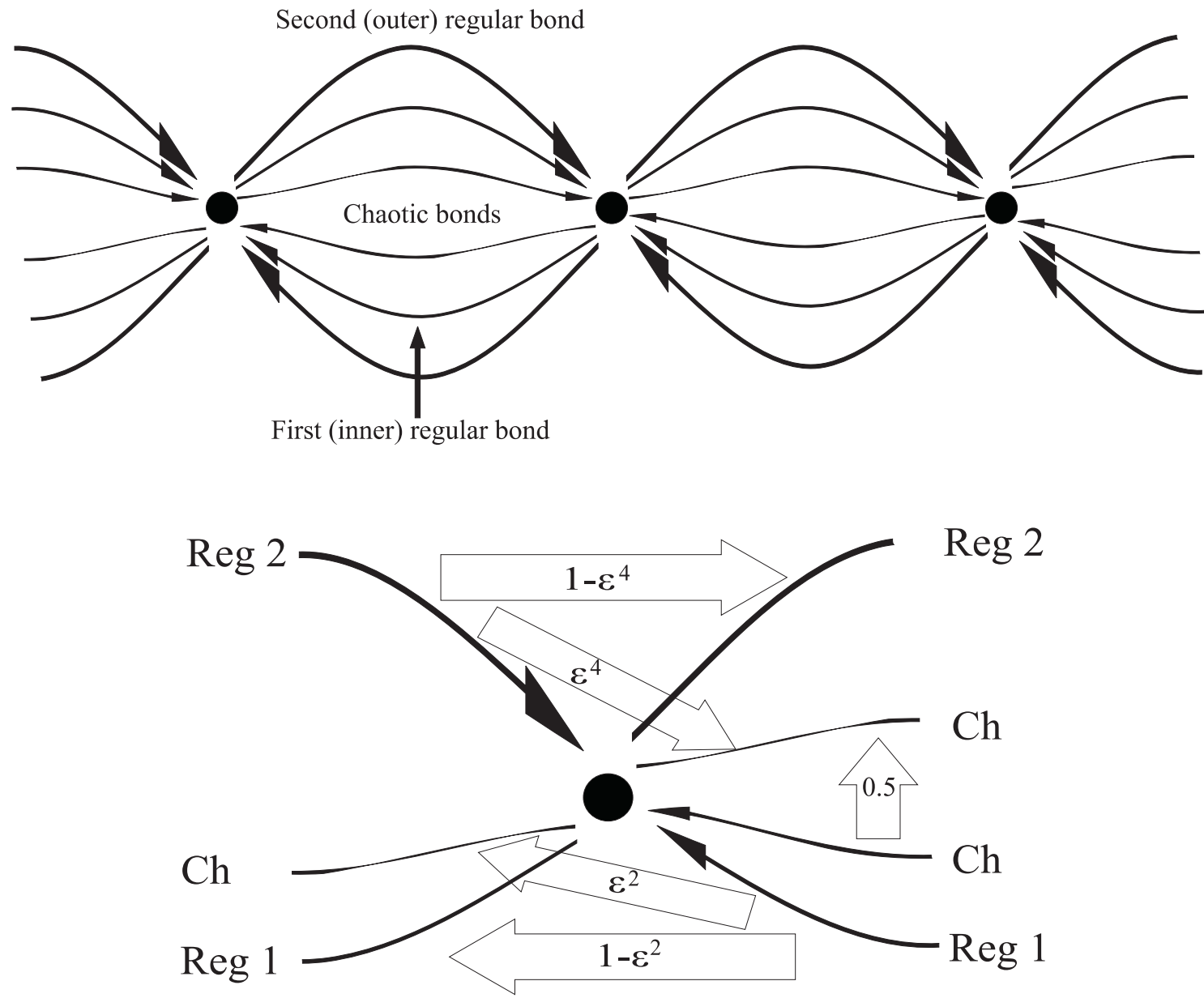

Figure 4.9: (top) Chain of "unit cells" of the graph toy model which corresponds to the standard map with accelerator modes. The situation where $A_{\text {island }} / h=2$ is modelled by two regular bonds in each direction. (bottom) Coupling rates of the bonds at one vertex; for clarity, only seven of the twelve bonds are shown. The chaotic bonds couple back to the regular ones with the same rates, $\epsilon^{2}$ and $\epsilon^{4}$, respectively.

In the second step, a unitary matrix $u$ is found, with coupling rates similar to those of $M$. Such a matrix $u$ can be obtained from the diagonalization of $M$ as

$$
u=\sum_{j} \psi_{j}^{*} \mathrm{e}^{-i \lambda_{j}} \psi_{j}
$$

where $M \psi_{j}=\lambda_{j} \psi_{j}$ (this procedure is analogous to the representation of a time-evolution operator in the basis of eigenstates of the corresponding Hamiltonian). Now, due to the symmetry of the graph, $u$ is symmetric and can again be decomposed into four equally 


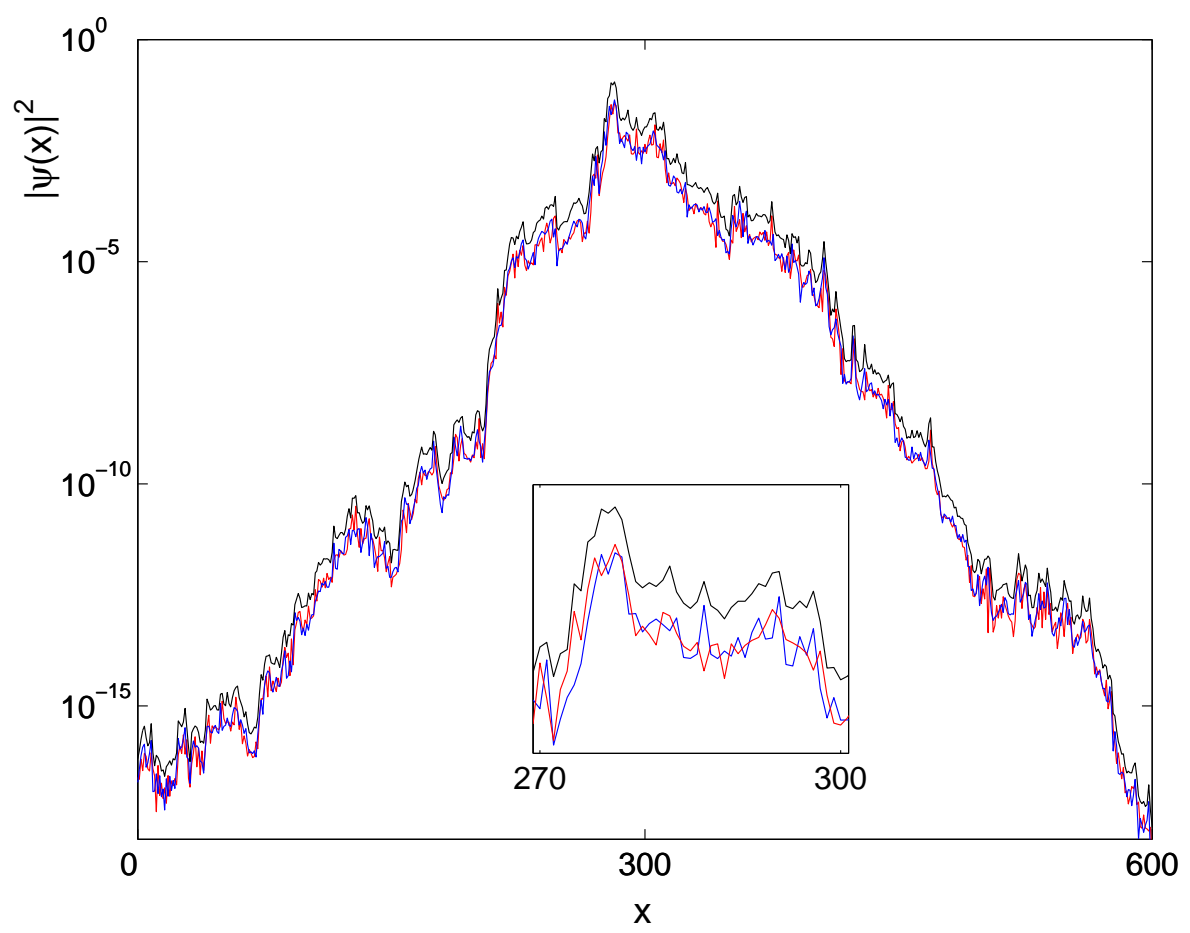

Figure 4.10: Weight of an eigenstate of $U$ for a graph with one level of regular bonds. The black line shows the total weight within one unit cell, blue is the weight on the chaotic and red is the weight on the regular bonds. As the inset indicates, the regular bonds are coupled strongly to the chaotic ones, leading to correlations in the weight distribution.

large quadratic blocks as,

$u=$\begin{tabular}{c|cc} 
& from left & from right \\
\hline to left & $R$ & $T$ \\
to right & $T$ & $R$
\end{tabular}.

This allows to set up the time-evolution operator $U$ for the chain of nodes. Here, the vertical order of the bond groups must equal the horizontal one. One can choose the sequence $(m-1)^{-},(m-1)^{+}, m^{-}, m^{+}, \ldots$, where the number denotes the "unit cell" and the superscript distinguishes between bonds directed to the left $(-)$ and to the right $(+)$. 


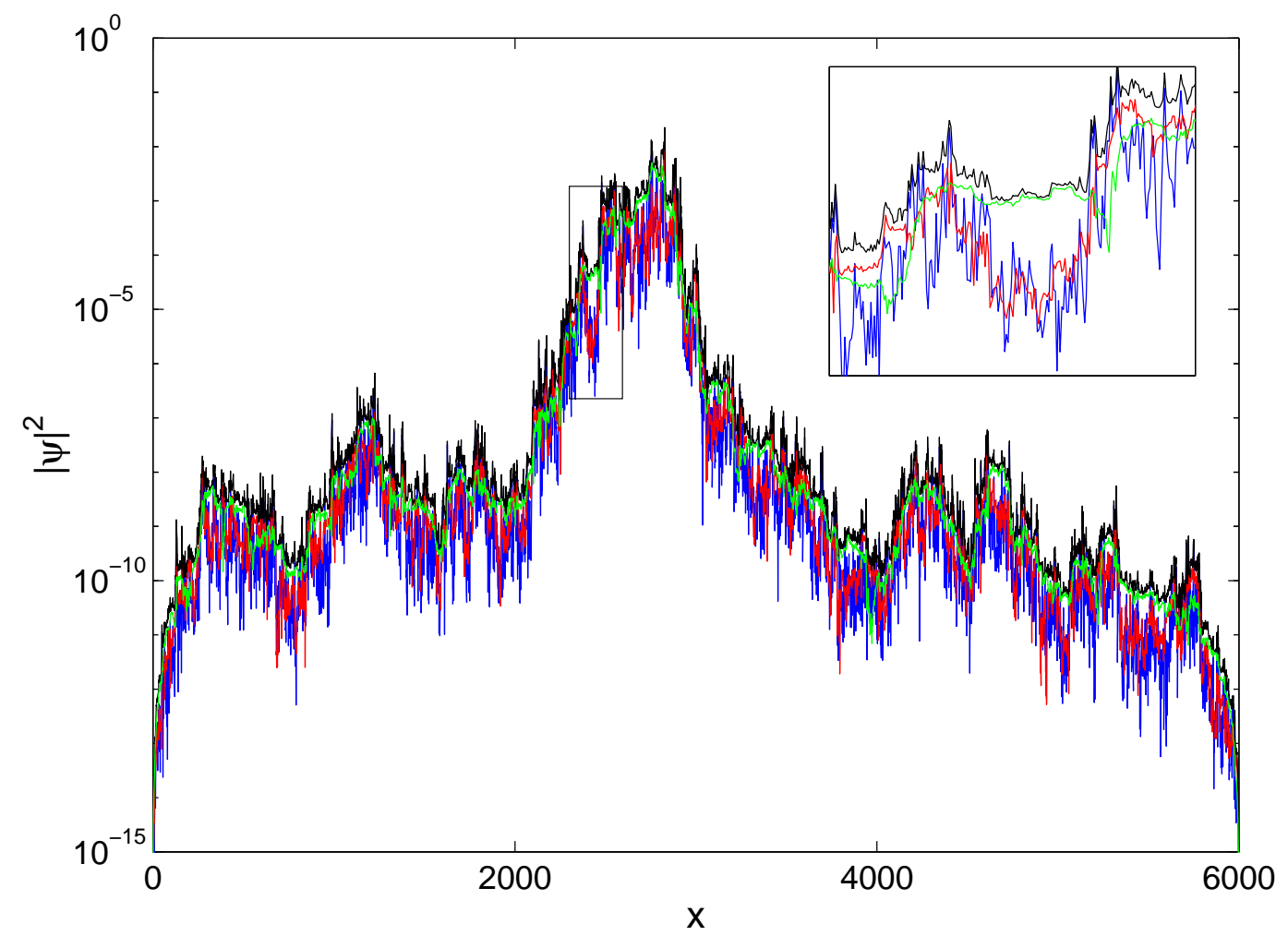

Figure 4.11: Weight distribution as in Fig. 4.10 but for a graph with two regular bonds. The weight in the additional bonds is shown in green, along with a magnification of a plateau (inset).

For this order, $U$ is a banded matrix given by

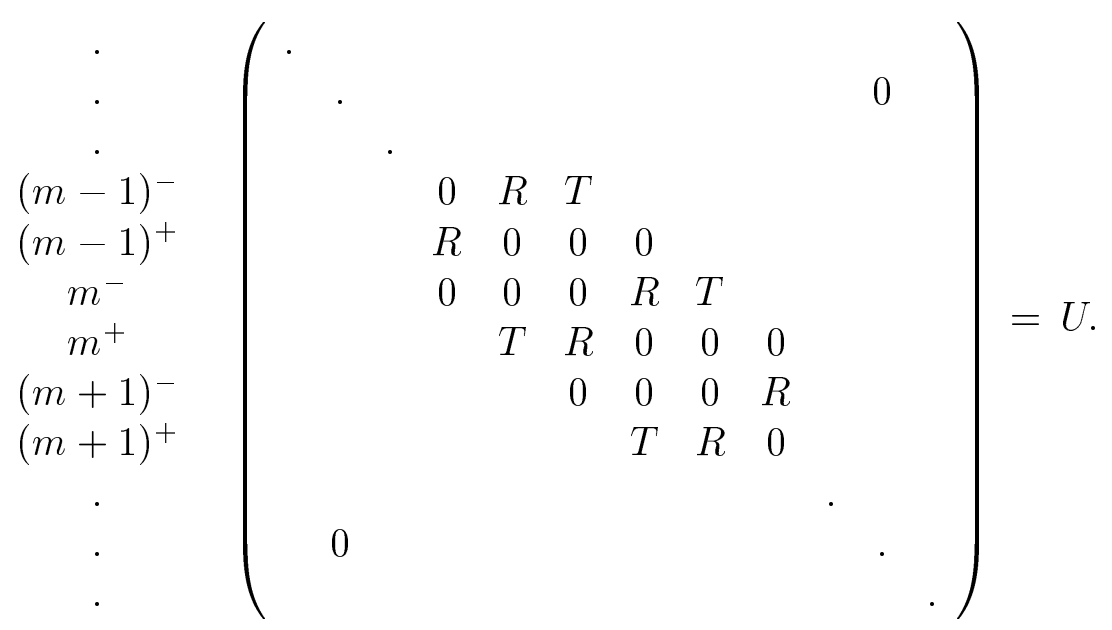

With the unitarity of $u, u \cdot u^{\dagger}=\mathbf{I}$, it is straightforward to verify that also $U \cdot U^{\dagger}=\mathbf{I}$, i.e. $U$ is again unitary. Finally, the disordering has to be modelled. This is achieved by the multiplication of $U$ with a diagonal matrix consisting of random phases: while the random phases neither affect the unitarity of $U$ nor the absolute square of its elements, i.e. the 
coupling rates, the periodicity of $U$ is broken, in analogy to $h \notin \mathbb{Q}$ for a kicked mapping.

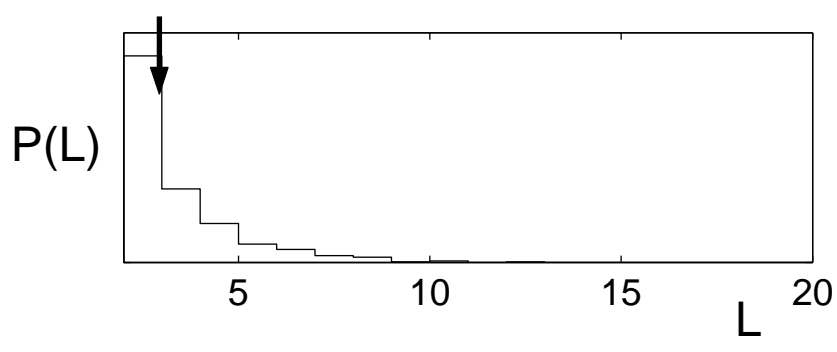

\title{
Plateau
}

lengths for

\author{
chaotic \\ bonds
}

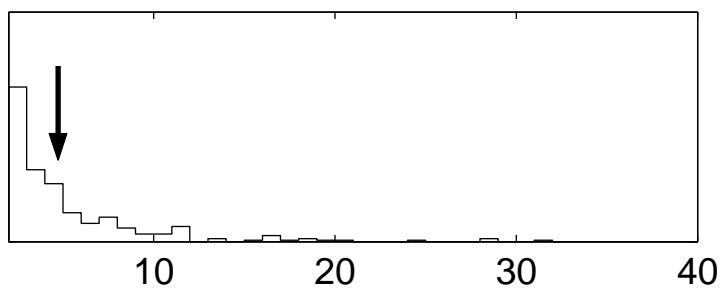

\section{inner \\ regular \\ bonds}

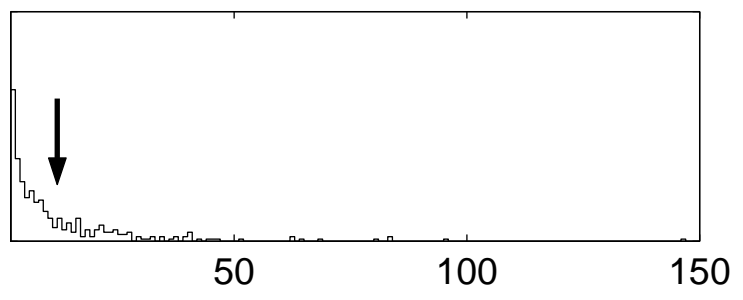

\section{outer regular bonds}

Figure 4.12: Distributions of plateau lengths. The arrows indicate the means $m$ of the distributions, $m_{\mathrm{ch}} \approx 0.958, m_{\mathrm{reg}_{1}} \approx 2.89$, and $m_{\mathrm{ch}} \approx 10.5$. This suggests an exponential scaling of the length with the hierarchy level.

The possibility to control the parameters $A_{\text {island }} / h$ through the number of regular bonds $l$ and $\gamma=\epsilon^{l}$ easily is the key reason for the use of graphs. Moreover, since the chaotic component of one unit cell is modelled by only two bonds, the width of the band of nonzero elements in $U$ is considerably smaller than for the time-evolution operator of a corresponding kicked map.

To show the analogy of this model to a Hamiltonian mapping, the eigenstates of a graph with one level of regular bonds and $\epsilon=0.4$ are calculated, see Fig. 4.10. The localization length is determined from an exponential fit as $\xi_{1} \approx 12.5$. While for a mapping, the weight of the state within a certain phase-space region has to be expressed by a Husimi function, this weight can trivially be read out from the eigenstate in the graph. One can also model the dynamical evolution of a wave packet initially localized within a regular island in one unit cell by choosing the initial state equal to 1 on a certain regular bond and 0 elsewhere. It can be seen that the dynamical localization length in this graph model again scales with the localization length of the eigenstates. For two regular bonds, an eigenstate is shown 
in Fig. 4.11. It complies to all the suggestions made above. The localization length is approximately increased by a factor $1 / \epsilon, \xi_{2} \approx 33.3$ and regular sections associated with the additional regular bond lead to long "plateaus" of appromitalely constant $\rho(x)$. In order to quantify this observation, one can define a plateau as a section of unit cells for which the weight distribution is dominated by the weight in bonds of the same hierarchy level. As shown in Fig. 4.12, the mean length of these plateaus indeed scales exponentially with the hierarchy level. This will be of use for the explanation of the shape of a saturated wave packet in Ch. 4.5.3.

\subsection{Wavepacket Dynamics in Disordered Hamiltonian Ratchets}

From the knowledge about stationary quantum mechanics in Hamiltonian ratchets gained in the last two sections, it is now possible to infer useful information about the dynamical evolution of a wave packet, since such a wave packet $\phi(x, t)$ can be written as a superposition of eigenstates $\psi_{\alpha}(x)$ as

$$
\phi(x, t)=\sum_{\alpha} c_{\alpha} \psi_{\alpha}(x) \mathrm{e}^{-2 \pi i \epsilon_{\alpha} t}
$$

The first subsection deals with the scaling of the operation time of a disordered quantum ratchet with $h$, which follows immediately from the scaling of the localization length. Since the calculation of the eigenstates with a large localization length is numerically very expensive, one has fallen back on other methods in the past [55-57,101-103]. A method which received a lot of attention is the measurement of the weight at the wave-packet front. The observation presented in the second subsection, however, strongly suggests that this quantity does not determine the shape of the localized wave packet. For the graph model introduced in Ch. 4.4.2, this shape is investigated and explained with the substructure of the eigenstates in the last subsection.

\subsubsection{Limit for Transport in Disordered Ratchets}

In the long-time limit any wave packet localizes and its velocity expectation value vanishes. From an experimental perspective, it is interesting to know the time scale on which this takes place, i.e., up to which a disordered Hamiltonian ratchet operates. Like in Ch. 3.2.2 for perfect quantum ratchets, one considers a wave packet $\phi(x, t)$ initially localized within one unit cell. Since the wave packet is given by the superposition of eigenstates Eq. (4.22), and with all the eigenstates showing the same localization length, the wave packet's localization length $\xi_{\phi}$ has the same scaling. Now, as was shown in Ch. 4.3.1, the localization 
length of the eigenstates in a ballistically transporting system scales exponentially with the inverse Planck's constant, $\xi_{\mathrm{T}} \sim \exp (C / h)$, where the constant $C$ depends on the system under consideration, leading to $\xi_{\phi} \sim \exp (C / h)$. With ballistic transport, the time scale on which localization takes place is proportional to the localization length, finally yielding $t_{\text {loc }} \sim \mathrm{e}^{C / h}$. This is confirmed numerically in the following.
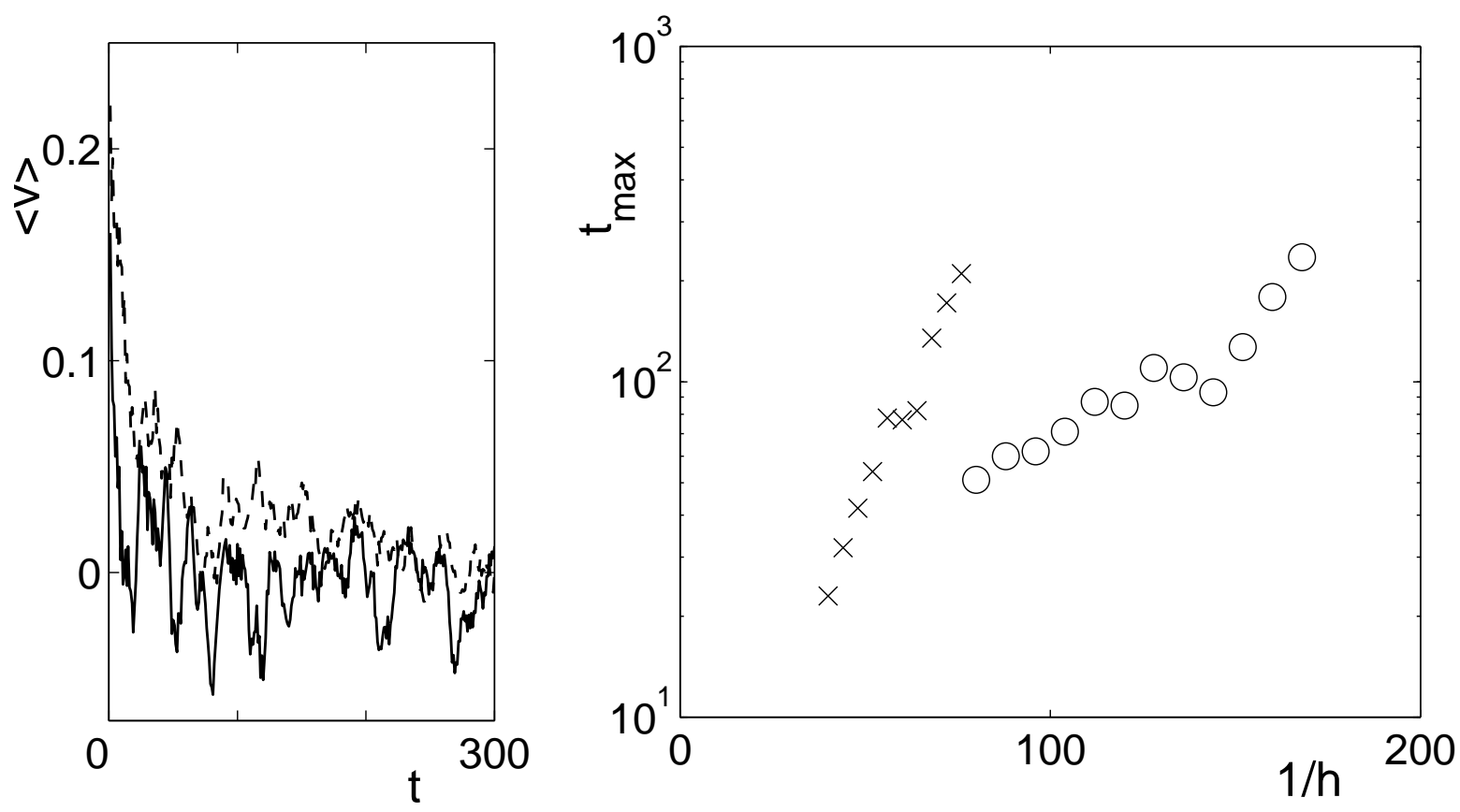

Figure 4.13: (left) Averaged velocity expectation values of wave packets initialized in the classically chaotic region at $\left(x_{0}, p_{0}\right)=(0,0.25 \pm 0.05)$ in the quantized system Eq. (2.31) for $h=1 /(8+\sigma)$ (solid line), and $h=1 /(16+\sigma)$ (dashed line). Beyond $t_{l o c}$, the velocity oscillates around zero. (right) Time $t_{\max }$ at which the averaged velocity expectation value falls short of a given limit under variation of $h$. Since the calculation time scales exponentially with $1 / h$, two different values for $v_{t h r}$ are chosen, namely $v_{\mathrm{thr}}=0.1$ (crosses), and $v_{\mathrm{thr}}=0.12$ (balls).

When the wave packet starts to localize, the velocity expectation value decreases. Hence, the maximum ratchet operation time $t_{\max }$ can be defined as the time at which the absolute value of this velocity crosses a given threshold value from above, $\left|\left\langle v_{\phi}\left(t_{\max }\right)\right\rangle\right| \equiv\left|v_{\phi, \text { thr }}\right|$, and $\left|\left\langle v_{\phi}\left(t<t_{\max }\right)\right\rangle\right|>\left|v_{\phi, \text { thr }}\right|$. Because of tunneling, the quantum transport cannot exceed the classical value. Therefore, the threshold velocity must be chosen smaller than the average velocity of a set of classical trajectories whose initial distribution mimics the Husimi representation of $\phi(x, 0)$ in phase space, $v_{\phi, \text { thr }}<v_{\phi \text {,class }}$. For the ratchet whose classical variant is given by Eq. (2.31), Fig. 4.5.1 shows the exponential scaling of the ratchet operation time with $1 / h$. It the semiclassical limit, therefore, the ratchet operation time diverges. 


\subsubsection{Dynamics at the Wave-Packet Front}

It has been a common method to derive information on the time evolution of a wave packet from the survival probability of weight in the front unit cell $P(t)=\left|\phi\left(w_{i} t, t\right)\right|^{2}$, with an initial wavepacket centered in unit cell $n_{0}=0[55,58]$. We have calculated this quantity for the ratchet given by Eq. (2.31), both for an irrational Planck's constant $h=1 /(n+\sigma)$ and for the rational $h=1 / n$. As indicated by Fig. 4.14, $P(t)$ does not depend on the rationality of $h$. This has been observed for various systems and values of the effective Planck's constant. This can be explained intuitively as follows: since there is essentially no weight beyond the unit cell $n=w_{i} t,\left|\phi\left(|n|>\left|w_{i} t\right|, t\right)\right|^{2} \approx 0$, no interference occurs such that the weight inside this unit cell is simply given by $P(t)=\mathrm{e}^{-\gamma t}$ in both cases (Fig. 4.14(b)). In order for this argument to hold, of course, the classical island under consideration must be the invariant set with the largest average velocity, since otherwise the weight from a "faster set" would lead to interferences.

For the remaining part of the wave packet, however, the shape depends crucially on the rationality of $h$ : for perfect quantum ratchets, the major part of the weight lags slightly behind the unit cell $n=w_{i}$ t, leading to a non-vanishing asymptotic transport, as explained in Ch. 3.2.3. In a disordered ratchet, however, the weight which has tunneled out starts to diffuse, leading to an isolated peak at the last unit cell [86], and, for larger times, to localization. Concluding, the observation that $P(t)$ does not depend on the rationality of $h$ is a strong indication that this quantity is not useful in the investigation of the full wave-packet dynamics. In particular, the assumption that $P(t)$ determines the asymptotic shape of the wave packet, $P(t) \sim \lim _{t \rightarrow \infty}\left|\phi\left(x / w_{i}\right)\right|^{2}$ [55], seems not to be valid since the asymptotic wave-packet shape depends crucially on this rationality.

\subsubsection{Asymptotic Wave-Packet Shape}

We now turn to the long-time limit of the probability distribution of a wave packet initialized within the classically chaotic region of one unit cell. Classically, Lévy walks (cf. Ch. 2.4) lead to anomalous diffusion of a bundle of chaotic trajectories, i.e. the diffusion process is enhanced due to the algebraically long ballistic walks in the hierarchical phase-space regions. For the standard map in a regime without accelerator modes, these regions were resolved in quantum calculations for one unit cell by Ketzmerick et al. [47]. It is required that the effective Planck's constant is smaller than the turnstile of a classical Cantorus. For systems with transporting islands, such a small $h$ would lead to a huge localization length which prohibits the calculation of eigenstates. Therefore, the quantum analogue to Lévy walks, which has been denoted as quantum anomalous diffusion [101], is not accessible by stationary quantum mechanics, and one has to fall back on the time evolution of wave packets. It has been shown by Stefancich et al. [55], that the weight in the front unit cell $P(t)$ of an initially chaotic wave packet exhibits a power-law decay 

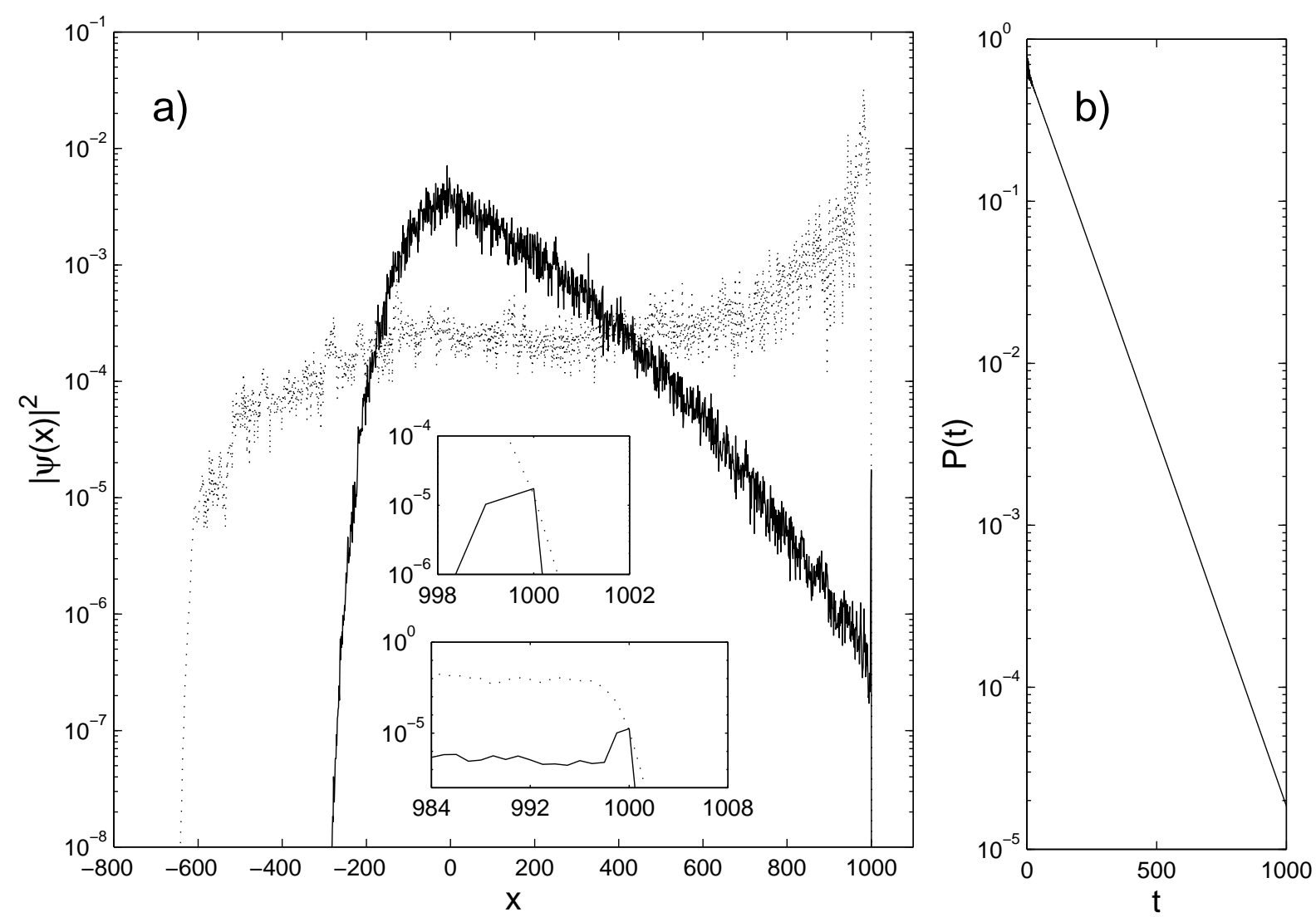

Figure 4.14: (a) Analogous wavepackets initially prepared in the regular island of the unit cell $n_{0}=0$ at time $t=1000$ for $h=1 / 16$ (dotted line) and $h=1 /(16+\sigma)$ (solid line) in the quantum version of the system Eq. 2.31 with smoothed potential. The classical probability would be restricted to the unit cell $n=1000$, whereas the quantum wavepacket in the system with rational $h$ has spread and lags slightly behind due to tunneling (cf. Ch. 3.2.3). The wavepacket in the disordered system shows an isolated peak at the unit cell $n=t$ with a value identical to that in the perfect ratchet (insets). (b) Due to tunneling, the quantum probability $P(t)$ in the unit cell $n=t$ decays exponentially as a function of time, with identical slopes for both the wavepacket in the disordered and in the perfect ratchet.

followed by an exponential decay for large times. It has been assumed that this is an indication of the quantum signatures of Lévy walks However, as will be shown in the following for the asymptotic weight distribution of such a wave packet, the power-law decay can be explained with the local structure of the eigenstates alone.

The toy model graph introduced in Ch. 4.4 .2 can once again be employed to decrease the numerical expense. Fig. 4.5.3 shows localized wave packets with a saturated variance for a maximum of three hierarchy levels. According to the observation, the shape of $|\phi(x, \infty)|^{2}$ can be separated into three regions: A plateau of approximately constant probability the length of which increases exponentially with $1 / h$, followed by a section with power-law 


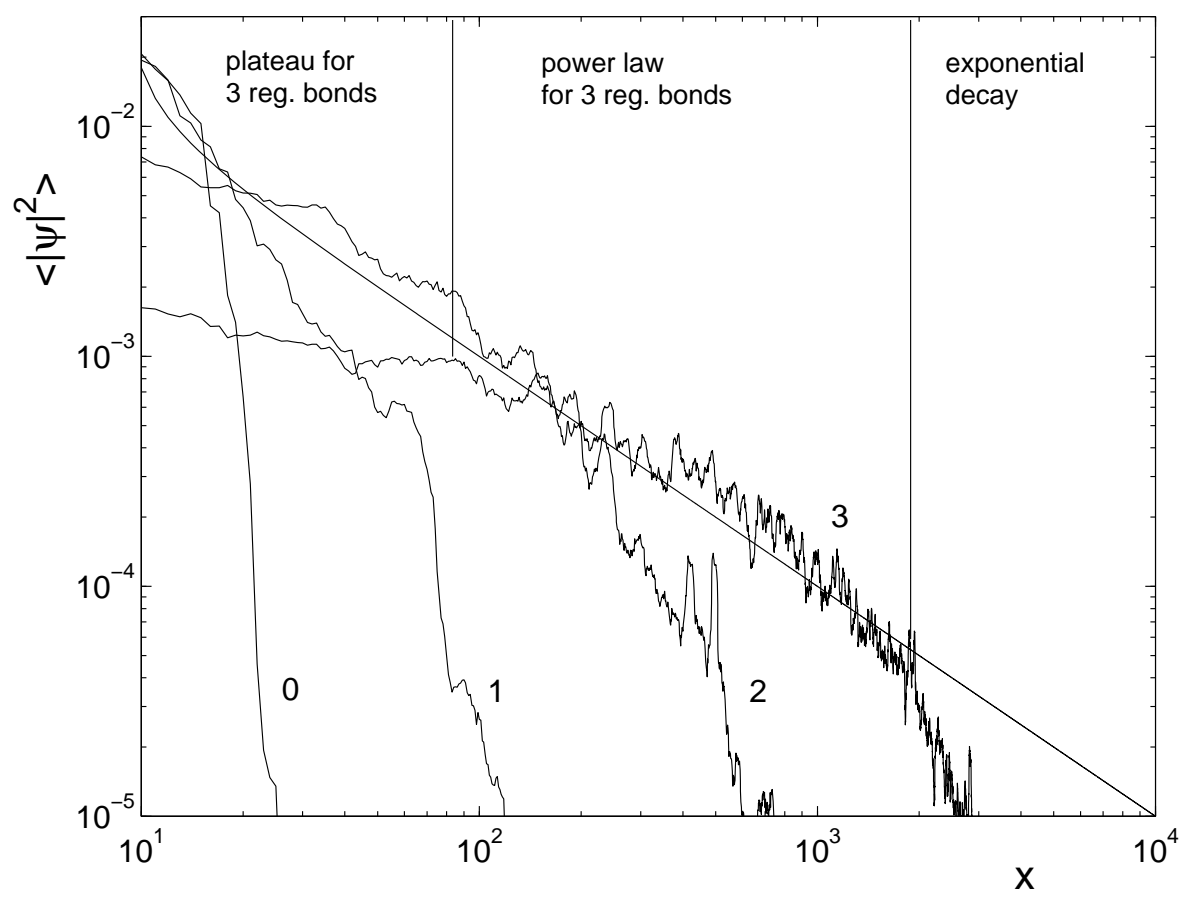

Figure 4.15: Localized wave packets which were initialized on a chaotic bond for graphs of different size. The numbers indicate the number of hierarchy levels of the corresponding model. For comparison, the curve proportional to $x^{-1}$ is shown. As indicated for the weight distribution in the graph with three regular bond levels, the shape can be divided into three sections.

decay which also extends exponentially, and finally, for large $x$, exponential decay. Whereas the last two regions would be expected from the semiclassical eigenfunction hypothesis, the plateau represents an evidence of its violation, as explained in the following.

According to this hypothesis, one has chaotic eigenstates and regular ones denoted as $\psi_{j}$, confined to the classical tori; $j$ labels the hierarchy level of the regular state with $\psi_{j_{\max }}$ being the state in the center of the island (see Fig. 4.16). The corresponding localization lengths $\xi_{j}$ would again be given by the inverse of the tunneling rate to the chaotic region $\gamma_{j}$ which in turn decreases exponentially with $j, \xi_{j}=1 / \gamma_{j} \sim \mathrm{e}^{-\mathrm{a} j}$ with some constant $a$ [86]. Then, the probability distribution of such regular eigenstates would be given by $\left|\psi_{j}(x)\right|^{2} \sim \mathrm{e}^{-\gamma_{j}|x|}$. Now, for large $x$, the asymptotic probability distribution of an initially chaotic wave packet $\phi(x, t)$ would be dominated by the shape of the regular eigenstates since the chaotic ones would localize on a much smaller scale, proportional to $1 / h$. The following analysis refers to large $x$, such that chaotic states can be neglected (symbolized by the approximation sign). It is natural to assume that the overlap of the initially chaotic wave packet $\phi$ with state $\psi_{j}$ is given by $\left|c_{j}\right|^{2}=\gamma_{j}$. In the long-time limit, this yields

$$
\lim _{t \rightarrow \infty}|\phi(x, t)|^{2} \approx \sum_{j}\left|c_{j}\right|^{2}\left|\psi_{j}(x)\right|^{2}=\sum_{j} \gamma_{j} \mathrm{e}^{-\gamma_{j}|x|}
$$



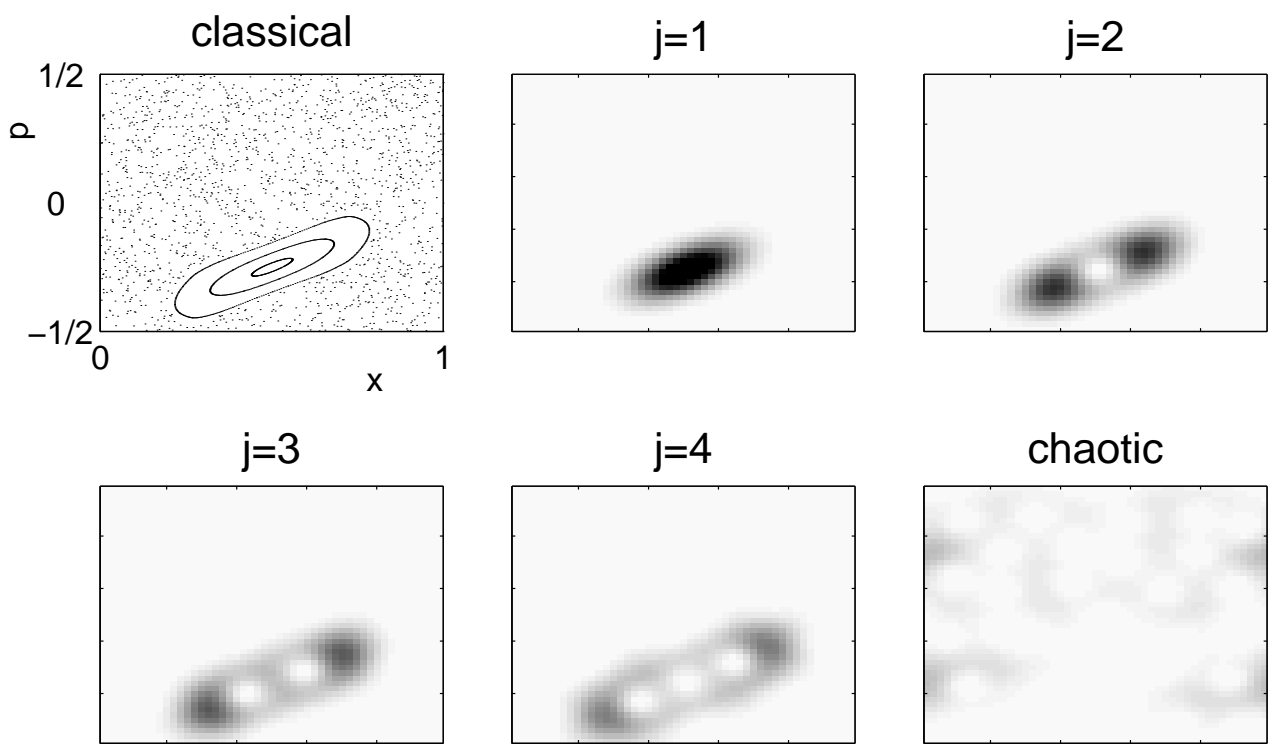

Figure 4.16: Eigenstates condensating on the classical tori in phase space, according to the semiclassical eigenfunction hypothesis. The state centered on the elliptic fixed point is given the maximum index. For comparison, the first subplot shows the Poincare section of the corresponding classical system Eq. (2.31).

where the approximation sign accounts for time-dependent fluctuations. In the semiclassical limit, the sum over $j$ can be approximated by an integral, $\int_{j=1}^{j_{\max }} \mathrm{d} j \gamma(j) e^{-|x| \gamma(j)}$, where $j \in\left[1, j_{\max }\right]$. With $\gamma_{j} \sim \mathrm{e}^{-\mathrm{a} j}$, one obtains by the substitution $\mathrm{d} j \rightarrow \mathrm{d} \gamma_{j}$,

$$
\begin{aligned}
\lim _{t \rightarrow \infty}|\phi(x, t)|^{2} & \approx \int_{j=1}^{j_{\max }} \mathrm{d} j \gamma(j) \mathrm{e}^{-\gamma(j)|x|} \\
& \sim \frac{1}{|x|}\left(\mathrm{e}^{-\gamma\left(j_{\max }\right)|x|}-\mathrm{e}^{-\gamma(1)|x|}\right) \approx \frac{1}{|x|} e^{-|x| \gamma\left(j_{\max }\right)},
\end{aligned}
$$

where the last approximation again improves for large $x$. The resulting distribution, a power law for comparatively small $x$ and an exponential decay thereafter, is shown in Fig. 4.17.

This shape is closely related to the observation for the asymptotic wave packets in the graph model Fig. 4.5.3, even though the semiclassical eigenfunction hypothesis does not apply. One can explain this with the local structure of the eigenfunctions of a symmetric quantum ratchet: the initial wave packet centered in the chaotic region of a certain unit cell excites those eigenstates exponentially weak which inside this unit cell exhibit a Husimi representation which resembles that of an imagined regular state $\psi_{j}$. On the other hand, the plateau of the asymptotic probability distribution can only be explained in terms of the violation of the semiclassical eigenfunction hypothesis. As has been discussed in Ch. 4.4, the eigenstates exhibit plateaus of approximately constant $|\psi(x)|^{2}$. These plateaus are confined 


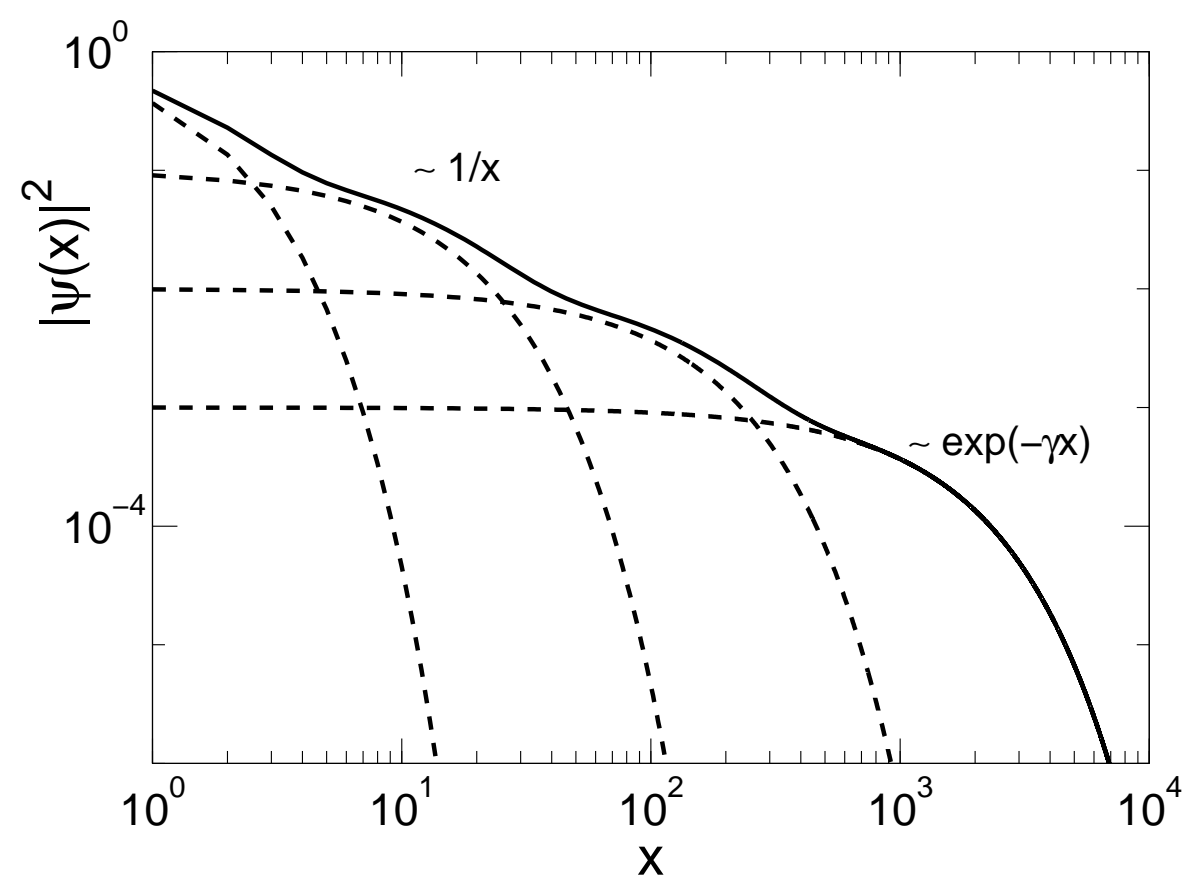

Figure 4.17: The superposition of exponentials (dashed lines) with exponentially small coefficients yields a power law decay with exponent -1 for a finite section according to Eq. 4.23. In the limit $x \rightarrow \infty$, the superposition approximates the curve with smallest negative exponent.

by peaks where the weight is concentrated in the chaotic region. A large excitations with one of these two peaks leads to a smearing of the asymptotic wave packet on the scale of the plateau length. This length scales exponentially with $1 / h$ (see. Fig. 4.12), which is transferred to the plateau in the spatial distribution of a saturated wave packet.

These statements could be confirmed by the calculation of localized wave packets deeper within the semiclassical limit. Since the observed eigenstates allow for even more involved phenomena of the dynamics, this might be a rewarding topic of further investigations. It can be concluded, however, that the observation of a power law in $P(t)$ is not an indication of the quantum signatures of Lévy walks but arises from the semiclassical substructure of the symmetric eigenstates alone.

\subsection{Outlook}

The failure of the semiclassical eigenfunction hypothesis and the appearance of amphibious eigenstates raise further questions: transporting island chains of arbitrary length exist even in systems with a finite phase space, such as in the neighborhood of the boundary circle enclosing a regular island. Will there be amphibious eigenstates? The statistical properties 
of chaotic eigenstates [81] can be seen in conductance measurements on quantum dots [104]. What are the statistical properties of amphibious eigenstates? Are they similar to those of chaotic eigenstates or are there specific correlations caused by the regular islands?

The recently developed techniques to observe atom dynamics in optical lattices $[105,106]$ are perfectly suited for the experimental study of amphibious eigenstates. In such systems wave packets can be prepared on selected points in phase space, for example on the center of an island, and their long-time dynamics can be studied. A measurement of the phase-space distribution would reveal that the asymptotic wave packet is uniformly distributed over phase space, independent of the initial preparation. This is in sharp contrast to a system with non-transporting islands and would be a clear experimental signature of amphibious eigenstates. 


\section{Chapter 5}

\section{Conclusion}

This thesis deals with directed transport and its implications in one-dimensional Hamiltonian systems that are periodic in space and time. It is shown that the coexistence of regular and chaotic motion as well as the breaking of time-reversal symmetry are required to achieve non-trivial directed chaotic transport both classically and quantum mechanically. Two basic results are obtained: A classical sum rule and its quantum analogon describe the ratchet mechanism in the absence of dissipation. The discovery of a new class of eigenstates modifies one of the fundamental assumptions of the quantum theory of nonlinear Hamiltonian systems. These findings lead to a number of corollary results.

The second chapter is concerned with the classical theory of Hamiltonian ratchets. It starts with a brief introduction to Hamiltonian mechanics, focussed on systems with a mixed phase space. The classical sum rule for ballistic transport is stated, which describes the relevant mechanism for the first time: directed transport, defined for the invariant manifolds as phase space volume times average velocity, is additive. Breaking time-reversal symmetry implies the desymmetrization of phase space. Hence, as long as the dynamics leave the different phase-space regions invariant, this causes the desymmetrization of transport in the dominant chaotic manifold. The sum rule is formulated both for the more general situation of a non-cyclic momentum variable, and for the simplified case. One arrives at the following implications: first, a desymmetrized mixed phase space is required and sufficient for chaotic directed transport. Second, the sum rule assists in the construction of optimized Hamiltonian ratchets with large transporting islands. This is shown explicitly for systems based on the standard map. Third, the dynamics within the manifolds only affect the shape of the velocity distribution, not the mean transport. The invariance of this mean under variation of the fraction of phase space supporting Lévy flights is shown also numerically.

In the following chapter, quantized Hamiltonian mappings are studied for the case where the quantization parameter fits exactly into the spatial period. In this situation, the 
Bloch theorem applies, leading to Bloch bands of eigenvalues. In terms of the winding numbers of these bands, which equal the velocity expectation values of the corresponding eigenfunctions, the quantum sum rule is formulated in analogy to the classical sum rule for compact phase space: the sum over all band slopes vanishes identically, as is the case for the non-trivial transport of the union of all the classical phase space regions. It is shown that quantum transport of these perfect quantum ratchets persists even in the long time limit, which is counterintuitive. The new aspect of quantum mechanics, i.e. tunneling across classical barriers, is shown to correspond to the avoided crossings of the bands. In terms of these narrowly avoided crossings, the coexistence of quantum tunneling with asymptotic transport is explained. In addition to the traditional WKB approximation, the analysis yields a new understanding of the exponential decay of the probability within the transporting island. For short times, the two-point correlation of the spectrum is shown to contain all the dynamical information of the system, in agreement with semiclassics. The form factor, a double Fourier transform of these correlations, is related analytically to the classical probability distributions. The investigation of the Chern numbers of the bands, however, leads to the insight that these numbers are not useful in the determination of directed transport.

The last chapter deals with the important case in which the ratio between the quantization parameter and the spatial period is irrational. In this case, the Bloch theory is not applicable, and quantum tunneling will vitiate transport for long times. The stationary states of Hamiltonian ratchets are shown to ignore the classical phase space structure completely, by comparing their Husimi representation with the classical Poincaré section. It is argued that this property persists even in the semiclassical limit. Such a rigorous violation of the semiclassical eigenfunction hypothesis has not been stated previously. Although in systems with broken time-reversal symmetry the steady states contradict the condensation hypothesis even locally, this is not the case for the standard map. The numeric computation for the latter case is carried out for a mapping and a quantum graph, respectively, both of which being analogous to the standard map. Since the appropriate construction of such a graph assumes a correct paradigm of the physical situation, the agreement of the results obtained from it with those of the mapping support the explanation given for amphibious eigenstates. In particular, this explanation is based on perturbation theory for the coupling constant of chaotic and regular regions. The analysis shows that the semiclassical eigenfunction hypothesis applies as long as the relevant system size, is smaller than a critical value, which is determined by the localization length. These findings can be transferred to the study of the time evolution of an initially localized wave packet. The quantity previously considered to be a good approximation to the asymptotic wave packet shape, the survival probability for the weight inside the transporting island $P(t)$, is found not to depend on the rationality of the quantization parameter. Since the dynamical properties, however, depend crucially on this rationality, the investigation of $P(t)$ is not meaningful. Instead, the time evolution must either be studied in terms of eigenstates or carried out for the whole wave packet. It is shown that both approaches lead to an asymptotic weight distribution with a shape of the superposition of a power law decay and an exponential decay. 
It has been suggested previously that such a power law decay is the quantum signature of Lévy flights. This is excluded for this study, however, since the classical phase-space regions responsible for the flights are not resolved by the effective Planck's constant. 


\section{Appendix A}

\section{Generalization of the Hellman-Feynman Theorem}

\section{A.1 Hellman-Feynman Theorem for Time-Periodic Sys- tems}

It will be shown that the velocity expectation value of an eigenstate $\psi_{\alpha, k}$ with quasienergy $\epsilon_{\alpha}(k)$ is given by

$$
v_{\alpha, k}=\frac{\mathrm{d} \epsilon_{\alpha}(k)}{\mathrm{d} k}
$$

In the first step, the band slope on the right-hand side is related to the expectation value of the derivative of the Bloch-phase dependent Hamiltonian in the eigenbasis of this Hamiltonian,

$$
\begin{aligned}
& \frac{1}{h} \frac{\mathrm{d} \epsilon_{\alpha}(k)}{\mathrm{d} k}=\frac{\mathrm{d} \varepsilon_{\alpha}(k)}{\mathrm{d} k}=\frac{\mathrm{d}}{\mathrm{d} k} \int_{0}^{1} \mathrm{~d} t \int_{0}^{1} \mathrm{~d} x u_{\alpha, k}^{*} \hat{H}(k) u_{\alpha, k} \\
& =\int_{0}^{1} \mathrm{~d} t \int_{0}^{1} \mathrm{~d} x u_{\alpha, k}^{*} \frac{\mathrm{d} \hat{H}(k)}{\mathrm{d} k} u_{\alpha, k} \\
& +\int_{0}^{1} \mathrm{~d} t \int_{0}^{1} \mathrm{~d} x \frac{\mathrm{d} u_{\alpha, k}^{*}}{\mathrm{~d} k} \hat{H}(k) u_{\alpha, k}+\int_{0}^{1} \mathrm{~d} t \int_{0}^{1} \mathrm{~d} x u_{\alpha, k}^{*} \hat{H}(k) \frac{\mathrm{d} u_{\alpha, k}}{\mathrm{~d} k} \\
& =\int_{0}^{1} \mathrm{~d} t \int_{0}^{1} \mathrm{~d} x u_{\alpha, k}^{*} \frac{\mathrm{d} \hat{H}(k)}{\mathrm{d} k} u_{\alpha, k}+\epsilon_{\alpha}(k) \frac{\mathrm{d}}{\mathrm{d} k} \int_{0}^{1} \mathrm{~d} t \int_{0}^{1} \mathrm{~d} x u_{\alpha, k}^{*} u_{\alpha, k} \\
& =\int_{0}^{1} \mathrm{~d} t \int_{0}^{1} \mathrm{~d} x u_{\alpha, k}^{*} \frac{\mathrm{d} \hat{H}(k)}{\mathrm{d} k} u_{\alpha, k}+0 .
\end{aligned}
$$


This is the generalization of the Hellman-Feynman theorem [107] to time-periodic systems as stated by Sambe [79]. For a Hamiltonian of the standard form Eq. (2.6),

$$
\frac{\mathrm{d} \hat{H}(k)}{\mathrm{d} k}=\frac{\mathrm{d} \hat{T}(\hat{p}+h k)}{\mathrm{d} k}=h \hat{T}^{\prime}(\hat{p}+h k),
$$

where the prime denotes taking the derivative with respect to the argument, and the factor $h$ stems from the inner derivative. Hence,

$$
\frac{\mathrm{d} \epsilon_{\alpha}(k)}{\mathrm{d} k}=\left\langle\left\langle u_{\alpha, k}\left|\hat{T}^{\prime}(\hat{p}+h k)\right| u_{\alpha, k}\right\rangle\right\rangle
$$

\section{A.2 The Unitary Transformation of the Kinetic En- ergy}

It remains to be shown that the eigenvalues of $\hat{T}^{\prime}(\hat{p}+h k)$ in the basis of $k$-independent states equal those of $\hat{T}^{\prime}(\hat{p})$ in the basis of $k$-dependent ones, namely that

$$
\int_{0}^{1} \mathrm{~d} t \int_{0}^{1} \mathrm{~d} x u_{\alpha, k}^{*} \hat{T}^{\prime}(\hat{p}+h k) u_{\alpha, k}=\int_{0}^{1} \mathrm{~d} t \int_{0}^{1} \mathrm{~d} x \psi_{\alpha, k}^{*} \hat{T}^{\prime}(\hat{p}) \psi_{\alpha, k} .
$$

This is done by expanding $\hat{T}^{\prime}$ as a power series of the argument on both sides and comparing the contributions to the sum. The factors in the Taylor expansion which do not depend on the argument are equal on both sides and can be neglected. Then, the $m^{\text {th }}$ summands read

$$
\begin{aligned}
\int_{0}^{1} \mathrm{~d} t \int_{0}^{1} \mathrm{~d} x u_{\alpha, k}^{*}\left(\frac{\hbar}{i} \frac{\mathrm{d}}{\mathrm{d} x}+h k\right)^{m} u_{\alpha, k} & =\int_{0}^{1} \mathrm{~d} t \int_{0}^{1} \mathrm{~d} x \psi_{\alpha, k}^{*}\left(\frac{\hbar}{i} \frac{\mathrm{d}}{\mathrm{d} x}\right)^{m} \psi_{\alpha, k} \Leftrightarrow \\
\int_{0}^{1} \mathrm{~d} t \int_{0}^{1} \mathrm{~d} x u_{\alpha, k}^{*}\left(\frac{\hbar}{i} \frac{\mathrm{d}}{\mathrm{d} x}+h k\right)^{m} u_{\alpha, k} & =\int_{0}^{1} \mathrm{~d} t \int_{0}^{1} \mathrm{~d} x u_{\alpha, k}^{*} \mathrm{e}^{-2 \pi i k x}\left(\frac{\hbar}{i} \frac{\mathrm{d}}{\mathrm{d} x}\right)^{m} \mathrm{e}^{2 \pi i k x} u_{\alpha, k}
\end{aligned}
$$

where $\hbar=h / 2 \pi$. This is fulfilled if

$$
\left(\frac{\hbar}{i} \frac{\mathrm{d}}{\mathrm{d} x}\right)^{m} \mathrm{e}^{2 \pi i k x} u_{\alpha, k}=\mathrm{e}^{2 \pi i k x}\left(\frac{\hbar}{i} \frac{\mathrm{d}}{\mathrm{d} x}+h k\right)^{m} u_{\alpha, k} .
$$

On the left-hand side, the $m^{\text {th }}$ derivative of a product of functions is calculated according to the Leibniz product rule as

$$
\begin{aligned}
\left(\frac{\hbar}{i} \frac{\mathrm{d}}{\mathrm{d} x}\right)^{m} \mathrm{e}^{2 \pi i k x} u_{\alpha, k} & =\sum_{j=0}^{m}\left(\begin{array}{c}
m \\
j
\end{array}\right)\left[\left(\frac{\hbar}{i} \frac{\mathrm{d}}{\mathrm{d} x}\right)^{m-j} \mathrm{e}^{2 \pi i k x}\right] \cdot\left[\left(\frac{\hbar}{i} \frac{\mathrm{d}}{\mathrm{d} x}\right)^{j} u_{\alpha, k}\right] \\
& =\mathrm{e}^{2 \pi i k x} \sum_{j=0}^{m}\left(\begin{array}{c}
m \\
j
\end{array}\right)\left(\frac{\hbar}{i} 2 \pi i k\right)^{m-j}\left[\left(\frac{\hbar}{i} \frac{\mathrm{d}}{\mathrm{d} x}\right)^{j} u_{\alpha, k}\right]
\end{aligned}
$$


The binomial operator on the right-hand side of Eq. (A.2) can be expanded to give

$$
\left(\frac{\hbar}{i} \frac{\mathrm{d}}{\mathrm{d} x}+h k\right)^{m}=\sum_{j=0}^{m}\left(\begin{array}{c}
m \\
j
\end{array}\right)(h k)^{m-j}\left(\frac{\hbar}{i} \frac{\mathrm{d}}{\mathrm{d} x}\right)^{j},
$$

which completes the proof. Together, Apps. A.1 and A.2 show that

$$
\left\langle\left\langle\psi_{\alpha, k}\left|\hat{T}^{\prime}(\hat{p})\right| \psi_{\alpha, k}\right\rangle\right\rangle=\mathrm{d} \epsilon_{\alpha}(k) / \mathrm{d} k .
$$




\section{Appendix B}

\section{Asymptotic Wavepacket Transport}

In this appendix, the average position

$$
x_{\mathrm{tr}}(t)=\int_{0}^{1} \mathrm{~d} \xi \sum_{n=-\infty}^{+\infty} \phi^{*}(\xi+n, t) n \phi(\xi+n, t)
$$

of the wavepacket $\phi(x, t)$ is calculated in the long-time limit $t \gg 1$. First, the wavepacket is written in the basis of eigenstates $\psi_{\alpha, k}(x, t)=\psi_{\alpha, k}(\xi+n, t)=\psi_{\alpha, k}(\xi, t) \mathrm{e}^{2 \pi i\left(k n-\epsilon_{\alpha, k} t\right)}$,

$$
\phi(x, t)=\sum_{\alpha} \int_{0}^{1} \mathrm{~d} k c_{\alpha, k} \psi_{\alpha, k}(\xi) \mathrm{e}^{2 \pi i\left(k n-\epsilon_{\alpha, k} t\right)},
$$

where

$$
c_{\alpha, k}=\int_{-\infty}^{+\infty} \mathrm{d} x \psi_{\alpha, k}^{*}(x) \phi(x, 0) .
$$

Recognizing that the integrand in Eq. (B.1) is periodic in $k$,

$$
\begin{aligned}
0 & =\int_{0}^{1} \mathrm{~d} k \frac{\mathrm{d}}{\mathrm{d} k}\left(c_{\alpha, k} \psi_{\alpha, k}(\xi) \mathrm{e}^{2 \pi i\left(k n-\epsilon_{\alpha, k} t\right)}\right) \\
& =\int_{0}^{1} \mathrm{~d} k \mathrm{e}^{2 \pi i\left(k n-\epsilon_{\alpha, k} t\right)} \frac{\mathrm{d}}{\mathrm{d} k}\left(c_{\alpha, k} \psi_{\alpha, k}(\xi)\right)+\int_{0}^{1} \mathrm{~d} k(2 \pi i n) \mathrm{e}^{2 \pi i\left(k n-\epsilon_{\alpha, k} t\right)} c_{\alpha, k} \psi_{\alpha, k}(\xi) \\
& -\int_{0}^{1} \mathrm{~d} k\left(2 \pi i \frac{\mathrm{d} \epsilon_{\alpha, k}}{\mathrm{~d} k} t\right) \mathrm{e}^{2 \pi i\left(k n-\epsilon_{\alpha, k} t\right)} c_{\alpha, k} \psi_{\alpha, k}(\xi),
\end{aligned}
$$

for all $\alpha$. Therefore,

$$
\begin{aligned}
n \phi(x, t) & =\sum_{\alpha} \int_{0}^{1} \mathrm{~d} k n \mathrm{e}^{2 \pi i\left(k n-\epsilon_{\alpha, k} t\right)} c_{\alpha, k} \psi_{\alpha, k}(\xi) \\
& =\sum_{\alpha} \int_{0}^{1} \mathrm{~d} k\left(\frac{\mathrm{d} \epsilon_{\alpha, k}}{\mathrm{~d} k} t \mathrm{e}^{2 \pi i k n} c_{\alpha, k} \psi_{\alpha, k}(\xi, t)-\frac{\mathrm{d}}{\mathrm{d} k}\left(c_{\alpha, k} \psi_{\alpha, k}(\xi) \mathrm{e}^{2 \pi i\left(k n-\epsilon_{\alpha, k} t\right)}\right)\right) .
\end{aligned}
$$


In the long-time limit, the first summand in the integrand in the last line becomes much larger than the second one, which will be neglected henceforth. Then, writing also the complex conjugate $\phi^{*}(x, t)$ as a superposition of eigenstates, one has

$$
x_{\operatorname{tr}}(t)=t \int_{0}^{1} \mathrm{~d} \xi \sum_{\alpha, \alpha^{\prime}} \int_{0}^{1} \mathrm{~d} k \int_{0}^{1} \mathrm{~d} k^{\prime} \frac{\mathrm{d} \epsilon_{\alpha, k}}{\mathrm{~d} k} c_{\alpha^{\prime}, k^{\prime}}^{*} \psi_{\alpha^{\prime}, k^{\prime}}^{*}(\xi, t) c_{\alpha, k} \psi_{\alpha, k}(\xi, t) \sum_{n=-\infty}^{+\infty} \mathrm{e}^{2 \pi i\left(k-k^{\prime}\right) n} .
$$

The sum over $n$ yields the periodic delta function,

$$
\sum_{n=-\infty}^{+\infty} \mathrm{e}^{2 \pi i\left(k-k^{\prime}\right) n}=\delta\left(\left(k-k^{\prime}\right) \bmod 1\right)
$$

but

$$
\delta\left(\left(k-k^{\prime}\right) \bmod 1\right)=\delta\left(k-k^{\prime}\right),
$$

since $k \in[0,1)$. This leads to

$$
x_{\mathrm{tr}}(t)=t \int_{0}^{1} \mathrm{~d} \xi \sum_{\alpha, \alpha^{\prime}} \int_{0}^{1} \mathrm{~d} k \frac{\mathrm{d} \epsilon_{\alpha, k}}{\mathrm{~d} k} c_{\alpha^{\prime}, k}^{*} \psi_{\alpha^{\prime}, k}^{*}(\xi, t) c_{\alpha, k} \psi_{\alpha, k}(\xi, t),
$$

whereas the orthonormalization of the eigenstates implies that

$$
x_{\mathrm{tr}}(t)=t \sum_{\alpha} \int_{0}^{1} \mathrm{~d} k\left|c_{\alpha, k}\right|^{2} \frac{\mathrm{d} \epsilon_{\alpha, k}}{\mathrm{~d} k} .
$$

Finally, with Eq. (3.13),

$$
x_{\mathrm{tr}}(t)=t \sum_{\alpha} \int_{0}^{1} \mathrm{~d} k\left|c_{\alpha, k}\right|^{2} v_{\alpha, k}
$$




\section{Appendix C}

\section{Generalized Form Factor for Periodic Maps in the Semiclassical Limit}

\section{C.1 Generalized Form Factor for a Regular Island}

Assuming that the subspectra which correspond to the classical finite invariant phasespace components are uncorrelated, one can decompose the form factor into a sum over form factors for each component. The generalized (i.e. unit-cell specific) form factor which corresponds to the finite invariant set $m$ is given by

$$
K_{n}^{(\mathrm{m})}(t)=h\left\langle\left|a_{n}^{(\mathrm{m})}(t)\right|^{2}\right\rangle
$$

with

$$
a_{n}^{(\mathrm{m})}(t)=\sum_{\alpha=1}^{N_{m}} \int_{0}^{1} \mathrm{~d} k \mathrm{e}^{+2 \pi \mathrm{i}\left(k n-\epsilon_{\alpha, k} t\right)},
$$

where the sum includes all the reconnected bands which pertain to the relevant subspectrum in the diabatic approximation (see Ch. 3.2). In this approximation, which improves in the semiclassical limit, the regular bands corresponding to an island $i$ are straight lines

$$
\epsilon_{\alpha}(k) \approx w_{i} k+c_{\alpha},
$$

where $w_{i}$ is the classical velocity of the island and the $c_{\alpha}=\epsilon_{\alpha}(0)$ are Poissonian distributed. Exploiting the straight-line type of the regular bands, one has

$$
\begin{aligned}
a_{n}^{(\text {reg })}(t) & =\sum_{\alpha=1}^{N_{i}} \int_{0}^{1} \mathrm{~d} k \exp \left[2 \pi \mathrm{i}\left(k\left(n-w_{i} t\right)+c_{\alpha} t\right)\right] \\
& =\delta_{n, w_{i} t} \sum_{\alpha=1}^{N_{i}} \mathrm{e}^{2 \pi i c_{\alpha} t} .
\end{aligned}
$$


Hence,

$$
K_{n}^{(\mathrm{reg})}(t)=h \delta_{n, w_{i} t}\left\langle\sum_{\alpha=1}^{N_{i}} \sum_{\alpha^{\prime}=1}^{N_{i}} \mathrm{e}^{2 \pi i\left(c_{\alpha}-c_{\alpha^{\prime}}\right) t}\right\rangle .
$$

The expression in the brackets is the usual form factor for a Poissonian ensemble which is simply given by the size of the spectrum $N_{i}$ [81] Ch. 4. In the semiclassical limit, the fraction of bands which correspond to the island equals the fraction of the island's volume in phase space, $\lim _{h \rightarrow 0}\left(N_{i} / N=N_{i} h\right)=f_{i}$. Then,

$$
K_{n}^{(\mathrm{reg})}(t)=f_{i} \delta_{n, w_{i} t},
$$

which is the classical probability $P_{n}^{(\mathrm{reg})}(t)$ of being in unit cell $n$ at time $t$ with an initial distribution confined to the regular island of unit cell $n_{0}$, at time $t_{0}=0$.

\section{C.2 Form Factor for the Chaotic Component}

While the special form of regular bands allows to obtain the classical limit easily by starting from Eq. (C.2), one has to evaluate the trace of the time evolution operator in the chaotic case. Assuming that the chaotic subset of the spectrum behaves like the spectrum of a fully chaotic system, one can fall back on the semiclassical analysis of maps by Tabor [108]. In the first step, we derive an expression for the classical distribution. Then, the form factor for the chaotic component is calculated in the semiclassical limit. Finally, the close relation to the classical distribution is shown.

Consider a classical map generated by the action for one time step $S\left(x_{1}, x_{0}\right)$, such that

$$
p_{0}=-\frac{\partial S\left(x_{1}, x_{0}\right)}{\partial x_{0}}, \quad p_{1}=\frac{\partial S\left(x_{1}, x_{0}\right)}{\partial x_{1}} .
$$

For $t$ time steps, the propagator is simply the sum over the individual steps,

$$
S\left(x_{t}, x_{0}, t\right)=\sum_{i=1}^{t} S\left(x_{i}, x_{i-1}\right) .
$$

For such a map, the classical density of a bundle of trajectories, initialized in a small phase-space volume around $x_{0}$, at time $t$ in a small volume around $x_{t}$ is given by

$$
C\left(x_{t}, x_{0}, t\right)=\left|-\frac{\partial^{2} S\left(x_{t}, x_{0}, t\right)}{\partial x_{t} \partial x_{0}}\right|,
$$

(see [64]). Once again decomposing the spatial coordinate $\left(x_{t}=\xi_{t}+n_{t}, x_{0}=\xi_{0}+n_{0}=\xi_{0}\right)$, the classical probability to be in unit cell $n$ at time $t$, upon starting out with the initial distribution described in App. C.1, is then given by

$$
P_{n}^{(\mathrm{ch})}(t) \sim \int_{\mathrm{ch}} \mathrm{d} \xi_{t} \mathrm{~d} \xi_{0} C\left(\xi_{t}+n_{t}, \xi_{0}, t\right) .
$$


Since the dynamics within (ch) is chaotic, the transition probability is effectively independent of $\xi_{0}$, which can be used to replace $\xi_{0}$ with $\xi_{t}$ :

$$
P_{n}^{(\mathrm{ch})}(t) \sim \int_{\mathrm{ch}} \mathrm{d} \xi_{t} C\left(\xi_{t}+n_{t}, \xi_{t}, t\right) \equiv \int_{\mathrm{ch}} \mathrm{d} \xi C(\xi+n, \xi, t) .
$$

For an initial distribution which is given by the characteristic function of the unit cell $n=0$, the weight in the chaotic sea is given by $f_{\mathrm{ch}}$. This determines the normalization of the classical distribution as $\sum_{n} P_{n}^{(\mathrm{ch})}(t)=f_{\mathrm{ch}}$.

Now, the calculation of the form factor starts with the evaluation of

$$
a_{n}^{(\mathrm{ch})}(t)=\int_{0}^{1} \mathrm{~d} k \mathrm{e}^{+2 \pi \mathrm{i} k n} \operatorname{tr}^{(\mathrm{ch})} \hat{U}_{k}^{t}=\int_{0}^{1} \mathrm{~d} k \mathrm{e}^{+2 \pi \mathrm{i} k n} \int_{\text {ch }} \mathrm{d} \xi\left\langle\xi\left|\hat{U}_{k}^{t}\right| \xi\right\rangle .
$$

The Bloch-phase dependent Floquet operator can be written in diagonal form as

$$
U_{k}^{t}=\sum_{\alpha}\left|\psi_{\alpha, k}\right\rangle \mathrm{e}^{-2 \pi i \epsilon_{\alpha}(k) t}\left\langle\psi_{\alpha, k}\right|
$$

leading to

$$
\left\langle\xi\left|\hat{U}_{k}^{t}\right| \xi\right\rangle=\sum_{\alpha} \psi_{\alpha, k}(\xi) \mathrm{e}^{-2 \pi i \epsilon_{\alpha}(k) t} \psi_{\alpha, k}^{*}(\xi) .
$$

Upon exchanging the order of integration,

$$
\begin{aligned}
a_{n}^{(\mathrm{ch})}(t) & =\int_{\mathrm{ch}} \mathrm{d} \xi \sum_{\alpha} \int_{0}^{1} \mathrm{~d} k \mathrm{e}^{+2 \pi \mathrm{i} k n} \psi_{\alpha, k}(\xi) \mathrm{e}^{-2 \pi i \epsilon_{\alpha}(k) t} \psi_{\alpha, k}^{*}(\xi) \\
& =\int_{\mathrm{ch}} \mathrm{d} \xi \sum_{\alpha} \int_{0}^{1} \mathrm{~d} k \psi_{\alpha, k}(\xi+n) \mathrm{e}^{-2 \pi i \epsilon_{\alpha}(k) t} \psi_{\alpha, k}^{*}(\xi) \\
& =\int_{\mathrm{ch}} \mathrm{d} \xi\left\langle\xi+n\left|\sum_{\alpha} \int_{0}^{1} \mathrm{~d} k\right| \psi_{\alpha, k}\right\rangle \mathrm{e}^{-2 \pi i \epsilon_{\alpha}(k) t}\left\langle\psi_{\alpha, k} \mid \xi\right\rangle \\
& =\int_{\text {ch }} \mathrm{d} \xi\left\langle\xi+n\left|\hat{U}^{t}\right| \xi\right\rangle .
\end{aligned}
$$

In the semiclassical limit one obtains as an approximation to the quantum propagator $\left\langle x_{t}\left|\hat{U}^{t}\right| x_{0}\right\rangle$ of a fully chaotic system in position representation the Van Vleck propagator

$$
\left\langle x_{t}\left|\hat{U}^{t}\right| x_{0}\right\rangle \approx \sum_{\beta} \sqrt{\frac{1}{i 2 \pi \hbar}\left|\frac{\partial^{2} S^{\beta}\left(x_{t}, x_{0}, t\right)}{\partial x_{t} \partial x_{0}}\right|} \exp \left[i\left(S^{\beta}\left(x_{t}, x_{0}, t\right) / \hbar-\nu^{\beta} \pi / 2\right)\right],
$$

where the sum includes all the classically allowed orbits $\beta$ from $x_{0}$ to $x_{t}$ and $\nu$ is the Morse index of that orbit [108]. Using this expression in Eq. (C.3) yields

$$
a_{n}^{(\mathrm{ch})}(t) \approx \int_{\mathrm{ch}} \mathrm{d} \xi \sum_{\beta} \sqrt{\frac{1}{i 2 \pi \hbar}\left|\frac{\partial^{2} S^{\beta}(\xi+n, \xi, t)}{\partial(\xi+n) \partial \xi}\right|} \exp \left[i\left(S^{\beta}(\xi+n, \xi, t) / \hbar-\nu^{\beta} \pi / 2\right)\right] .
$$


Upon insertion into Eq. (C.1), one obtains a double sum, over $\beta$ and $\beta^{\prime}$. The diagonal approximation $\left(\beta=\beta^{\prime}\right)$, the validity of which is restricted to times smaller than the Heisenberg time of the chaotic component, $t<t_{H}^{\text {ch }}=N_{\mathrm{ch}}$, yields

$$
\begin{aligned}
K_{n}^{(\mathrm{ch})}\left(t<t_{H}^{\mathrm{ch}}\right) & =h\left\langle\left|a_{n}^{(\mathrm{ch})}(t)\right|^{2}\right\rangle \\
& \approx g(t) h \int_{\mathrm{ch}} \mathrm{d} \xi C(\xi+n, \xi, t),
\end{aligned}
$$

where $g(t)$ is a time-dependent normalization constant which arises from the time dependence of the number of periodic orbits $\beta$. It can be determined from the study of orbit repetitions and the sum rule of Hannay and Ozorio de Almeida [81] Ch. 8: for the usual form factor, and with the scaled time $\tau=t / t_{H}^{\text {ch }}$, one has

$$
K\left(t<t_{H}^{\mathrm{ch}}\right)=\left\{\begin{array}{cc}
h N \tau & : \mathrm{CUE} \\
h N 2 \tau-\tau \log (1+2 \tau) & : \mathrm{COE}
\end{array} .\right.
$$

(The spectrum of maps with broken time-reversal symmetry corresponds to a unitary ensemble on the unit circle in the complex plane (CUE), whereas that for time-symmetric maps is given by an orthogonal ensemble (COE).) Since the shift from the extended system to the restricted phase space, i.e. from the usual to the generalized form factor, may not affect the normalization, one has also

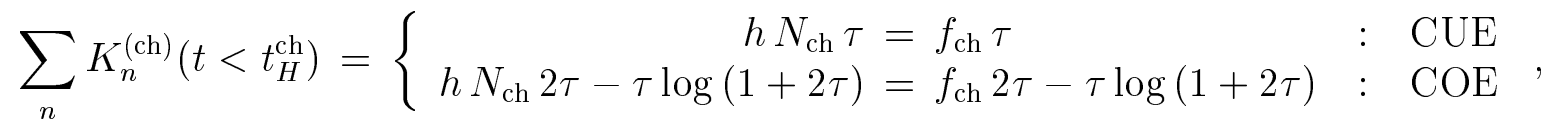

which determines the normalization constant in Eq. (C.5). Comparing with the classical probability finally yields

$$
K_{n, \mathrm{ch}}\left(t<t_{H}^{\mathrm{ch}}\right)=\left\{\begin{array}{cc}
\tau P_{n}^{(\mathrm{ch})}(t) & : \mathrm{CUE} \\
2 \tau-\tau \log (1+2 \tau) P_{n}^{(\mathrm{ch})}(t) & : \mathrm{COE}
\end{array} .\right.
$$

For a typical ratchet with broken time-reversal symmetry, one has the (CUE) case. 


\section{Bibliography}

[1] J. Maddox, Nature 365, 203 (1993).

[2] S. Leibler, Nature 370, 412 (1994).

[3] J. A. Spudich, Nature 372, 515 (1994).

[4] J. Rousselet, L. Salome, A. Ajdari, and J. Prost, Nature 370, 446 (1994).

[5] A. Lorke et al., Physica B 249, 312 (1998).

[6] H. Linke et al., Science 286, 2314 (1999).

[7] I. Zapata et al., Phys. Rev. Lett. 77, 2292 (1996).

[8] I. Derényi, C. Lee, and A.-L. Barabási, Phys. Rev. Lett. 80, 1473 (1998).

[9] C. Mennerat-Robilliard et al., Phys. Rev. Lett. 82, 851 (1999).

[10] C.-S. Lee et al., Nature 400, 337 (1999).

[11] A. Einstein, Ann. Phys. 19, 371 (1906).

[12] P. Langevin, Comptes Rendues 146, 530 (1908).

[13] M. von Smoluchowski, Phys. Z 13, 1069 (1912).

[14] R. P. Feynman, R. B. Leighton, and M. Sands, The Feynman Lectures on Physics (Addison-Wesley, Reading, MA, 1966), vol. 1, chap. 46.

[15] A. Ajdari and J. Prost, C. R. Acad. Sci. Paris 315, 1635 (1992).

[16] M. O. Magnasco, Phys. Rev. Lett. 71, 1477 (1993).

[17] R. D. Astumian and M. Bier, Phys. Rev. Lett. 72, 1766 (1994).

[18] C. R. Doering, W. Horsthemke, and J. Riordan, Phys. Rev. Lett. 72, 2984 (1994).

[19] M. O. Magnasco, Phys. Rev. Lett. 71, 1477 (1993). 
[20] A. Ajdari, D. Mukamel, L. Peliti, and J. Prost, J. Phys. I (France) 4, 1551 (1994).

[21] R. Bartussek, P. Hänggi, and J. G. Kissner, Europhys. Lett. 28, 459 (1994).

[22] P. Jung, J. G. Kissner, and P. Hänggi, Phys. Rev. Lett. 76, 3436 (1996).

[23] P. Reimann, M. Grifoni, and P. Hänggi, Phys. Rev. Lett. 79, 10 (1997).

[24] I. Goychuk, M. Grifoni, and P. Hänggi, Phys. Rev. Lett. 81, 649 (1998).

[25] P. Reimann and P. Hänggi, Chaos 8, 629 (1998).

[26] I. Goychuk and P. Hänggi, Europhys. Lett. 43, 503 (1998).

[27] S. Flach, O. Yevtushenko, and Y. Zolotaryuk, Phys. Rev. Lett. 84, 2358 (2000).

[28] I. Goychuk and P. Hänggi, Lecture Notes on Physics, Hrsg. J. Freund and T. Pöschel (Springer, Berlin, 2000), Vol. 557, pp. 7-20.

[29] H. Poincaré, Les méthodes nouvelles de la méchanique céleste Tome III. (GauthierVillars, Paris, 1899).

[30] A. N. Kolmogoroff, Dokl. Akad. Nauk. SSSR 98, 527 (1954).

[31] V. I. Arnold, Ups. Mat. Nauk. SSSR 18, 13 (1963).

[32] J. Moser, Nachr. Akad. Wiss. Göttingen 1, 15 (1962).

[33] L. Markus and K. R. Meyer, Memoirs of the American Mathematical Society (American Mathematical Society, Providence, 1974), Vol. 114.

[34] B. V. Chirikov, Phys. Rep. 52, 263 (1979).

[35] T. Geisel, in Lévy Flights and Related Phenomena in Physics., Hrsg. G. M. Zaslavsky (Springer, Berlin, 1995), section 4.

[36] O. Bohigas, M. J. Giannoni, and C. Schmit, Phys. Rev. Lett. 52, 1 (1984).

[37] M. L. Metha, Random Matrices (Academic Press, Boston, 1991).

[38] M. C. Gutzwiller, J. Math. Phys. 11, 1791 (1970).

[39] T. Dittrich, B. Mehlig, H. Schanz, and U. Smilansky, Phys. Rev. E 57, 359 (1998).

[40] I. C. Percival, J. Phys. B-At. Mol. Opt. Phys. 6, L229 (1973).

[41] M. V. Berry, Phil. Trans. R. Soc. A 287, 237 (1977).

[42] M. V. Berry, J. Phys. A 10, 2083 (1977). 
[43] M. V. Berry and M. Robnik, J. Phys. A: Math. Gen. 17, 2413 (1984).

[44] O. Bohigas, S. Tomsovic, and D. Ullmo, Phys. Rep. 223, 43 (1993).

[45] E. J. Heller and S. Tomsovic, Phys. Today 46, 38 (1993).

[46] T. Prosen and M. Robnik, J. Phys. A 26, 5365 (1993).

[47] R. Ketzmerick, L. Hufnagel, F. Steinbach, and M. Weiss, Phys. Rev. Lett. 85, 1214 (2000).

[48] P. W. Anderson, Phys. Rev. 109, 1492 (1958).

[49] P. W. Anderson, Rev. Mod. Phys. 50, 191 (1978).

[50] S. Fishman, D. R. Grempel, and R. E. Prange, Phys. Rev. Lett. 49, 509 (1982).

[51] F. L. Moore, J. C. Robinson, C. Bharucha, P. E. Williams, and M. G. Raizen, Phys. Rev. Lett. 73, 3974 (1994).

[52] J. C. Robinson, C. Bharucha, F. L. Moore, R. Jahnke, G. A. Georgakis, Q. Niu, , M. G. Raizen, and B. Sundaram, Phys. Rev. Lett. 74, 3963 (1995).

[53] F. L. Moore, J. C. Robinson, C. Bharucha, B. Sundaram, and M. G. Raizen, Phys. Rev. Lett. 75, 4598 (1995).

[54] T. Dittrich, Phys. Rep. 271, 267 (1996).

[55] M. Stefancich, P. Allegrini, L. Bonci, P. Grigolini, and B. J. West, Phys. Rev. E 57, 6625 (1998).

[56] B. Sundaram and G. M. Zaslavsky, Phys. Rev. E 59, 7231 (1999).

[57] A. Iomin and G. M. Zaslavsky, Chaos 10, 147 (2000).

[58] A. Iomin and G. M. Zaslavsky, Phys. Rev. E 63, 7203 (2001).

[59] B. G. Klappauf, W. H. Oskay, D. A. Steck, and M. G. Raizen, Phys. Rev. Lett. 81, 4044 (1998).

[60] H. Schanz, M. F. Otto, R. Ketzmerick, and T. Dittrich, Phys. Rev. Lett. 87, 070601 (2001).

[61] T. Dittrich, R. Ketzmerick, M. F. Otto, and H. Schanz, Ann. Phys. 9, 755 (2000).

[62] H. Schanz, M.-F. Otto, R. Ketzmerick, and T. Dittrich, in preparation.

[63] L. Hufnagel, R. Ketzmerick, M.-F. Otto, and H. Schanz, submitted. 
[64] M. C. Gutzwiller, Chaos in Classical and Quantum Mechanics (Springer, New York, 1990).

[65] A. J. Lichtenberg and M. A. Lieberman, Regular and Chaotic Dynamics (Springer, New York, 1992).

[66] J. M. Greene, J. Math. Phys. 9, 760 (1968).

[67] O. Yevtushenko, S. Flach, and K. Richter, Phys. Rev. E 61, 7215 (2000).

[68] P. G. Harper, Proc. Phys. Soc. London A 68, 874 (1955).

[69] D. R. Hofstadter, Phys. Rev. B 14, 2239 (1976).

[70] T. Geisel, R. Ketzmerick, and G. Petschel, Phys. Rev. Lett. 67, 3635 (1991).

[71] A. J. Lichtenberg and M. A. Lieberman, Regular and Chaotic Dynamics (Springer, New York, 1992), chap. 3.3.

[72] S. Denisov and S. Flach, 2001.

[73] B. Chirikov and D. L. Shepelyansky, Proceedings of the IXth Intern. Conf. on Nonlinear Oscillations (Princeton University Press, Princeton, 1983), no. PPPL-TRANS133.

[74] J. D. Meiss and E. Ott, Phys. Rev. Lett. 55, 2741 (1985).

[75] J. N. Mather, Topology 21, 457 (1982).

[76] I. C. Percival, AIP Conference Proceedings 57, 1179 (1980).

[77] S. Aubry, in Solitons and Condensed Matter Physics, Hrsg. A. R. Bishop and T. Schneider (Springer, Berlin, 1978), p. 264.

[78] J. D. Hanson, J. R. Cary, and J. D. Meiss, J. Stat. Phys. 99, 327 (1985).

[79] H. Sambe, Phys. Rev. A 7, 2203 (1973).

[80] J. von Neumann and E. P. Wigner, Phys. Z. 30, 467 (1929).

[81] F. Haake, Quantum Signatures of Chaos (Springer, New York, 1992).

[82] E. P. Wigner, Phys. Rev. 40, 749 (1932).

[83] K. Husimi, Proc. Phys. Math. Soc. Japan 22, 264 (1940).

[84] A. R. Kolovsky, S. Miyazaki, and R. Graham, Phys. Rev. E 49, 70 (1994).

[85] S. Miyazaki and A. R. Kolovsky, Phys. Rev. E 50, 910 (1994). 
[86] J. D. Hanson, E. Ott, and T. M. Antonsen, Phys. Rev. A 29, 819 (1984).

[87] T. Dittrich, B. Mehlig, H. Schanz, and U. Smilansky, Chaos, Solitons \& Fractals 8, 1205 (1997).

[88] J. H. Hannay and A. M. O. de Almeida, J. Phys. A 17, 3429 (1984).

[89] M. V. Berry, Proc. R. Soc. Lond. A 400, 229 (1985).

[90] R. B. Laughlin, Phys. Rev. B 23, 5632 (1981).

[91] R. E. Prange, Phys. Rev. B 23, 4802 (1981).

[92] D. J. Thouless, M. Kohmoto, M. P. Nightingale, and M. den Nijs, Phys. Rev. Lett. 49, 405 (1982).

[93] P. Leboeuf, J. Kurchan, M. Feingold, and D. P. Arovas, Phys. Rev. Lett. 65, 3076 (1990).

[94] P. Leboeuf, J. Kurchan, M. Feingold, and D. P. Arovas, Chaos 2, 125 (1992).

[95] S. Fishman, D. R. Grempel, and R. E. Prange, Phys. Rev. Lett. 49, 509 (1982).

[96] H. Furstenberg, Trans. Ann. Math. Soc. 108, 377 (1963).

[97] F. M. Izrailev, Phys. Rep. 196, 299 (1990).

[98] A. Voros, Ann. Inst. Henri Poincaré 24, 31 (1976).

[99] A. L. Shnirelman, Uspekhi Matem. Nauk. 29, 181 (1979).

[100] M. Robnik, Nonlinear Phenomena in Complex Systems 1, 1 (1998).

[101] R. Roncaglia, L. Bonci, B. J. West, and P. Grigolini, Phys. Rev. E 51, 5524 (1995).

[102] R. Bettin, R. Mannella, B. J. West, and P. Grigolini, Phys. Rev. E 51, 212 (1995).

[103] A. Iomin and G. M. Zaslavsky, Phys. Rev. E 60, 7580 (1999).

[104] Y. Alhassid, Rev. Mod. Phys. 72, 895 (2000).

[105] D. A. Steck, W. H. Oskay, and M. G. Raizen, Science 7293, 274 (2001).

[106] W. K. Hensinger et al., Nature 412, 52 (2001).

[107] R. P. Feynman, Phys. Rev. 56, 340 (1939).

[108] M. Tabor, Physica 6 D, 195 (1983). 


\section{Acknowledgements}

I would like to thank Prof. Dr. Theo Geisel for the acceptance to his group. His continuous dedication has created a stimulating and in equal measure friendly atmosphere.

Acknowledgment is due to Dr. Holger Schanz and Priv. Doz. Dr. Roland Ketzmerick whose permanent support and readiness for discussions was an indispensable prerequisite for this thesis.

I also acknowledge the numerous and fruitful discussions with Dr. Lars Hufnagel as well as Abigail Morrison's knowledge of the fine structure of the English language.

Dr. Wolf-Dieter Brandt, Dr. Denny Fliegner and Yorck-Fabian Temme essentially supported this work through their helpfulness in dealing with the computer system.

I also thank all the members of the group for a friendly environment: Steffen Arnrich, Rita Bartels, Oliver Bendix, Dmitri Bibitchkov, Dirk Brockmann, Michael Buschermöhle, Michael Denker, Markus Diesmann, Silke Dodel, Dr. Udo Ernst, Dr. Ragnar Fleischmann, Dr. Michael Herrmann, Matthias Kaschube, Dr. Tsampikos Kottos, Dr. Norbert Mayer, Björn Naundorf, Alexander Ossipov, Felix Petri, Dr. Hans-Ekkehard Plesser, Michael Schnabel, Dr. Dennis Springsguth, Dr. Frank Steinbach, Tom Tetzlaff, Marc Timme, Corinna Trautsch, Dr. Fred Wolf, Regina Wunderlich, and Alexander Zumdieck. 